Running Head: EMOTION REGULATION TYPES

\title{
A Data-Driven Typology of Emotion Regulation Profiles
}

João F. Guassi Moreira ${ }^{1 *}$, Razia S. Sahi ${ }^{1}$, Maria D. Calderon Leon ${ }^{1}$, Natalie M. Saragosa-Harris ${ }^{1}$, Yael H. Waizman $^{1}$, Anna E. Sedykin ${ }^{2}$, Emilia Ninova ${ }^{1}$, Tara S. Peris ${ }^{2}$, James J. Gross ${ }^{3}$, \& Jennifer A. Silvers ${ }^{1 *}$

${ }^{1}$ Department of Psychology, University of California, Los Angeles

${ }^{2}$ Division of Child \& Adolescent Psychiatry, Semel Institute for Neuroscience \& Human Behavior, University of California, Los Angeles

${ }^{3}$ Department of Psychology, Stanford University

*corresponding authors

A191 Franz Hall

502 Portola Plaza

Los Angeles CA, 90095-1563

jguassimoreira@ucla.edu

silvers@ucla.edu

\section{Author Contributions}

JFGM and JAS developed research questions and designed the study concept. Novel questionnaire measure (E-ERQ) items were developed by JFGM and JAS with critical feedback from RSS, MDCL, and YHW. Data were collected by JFGM, RSS, MDCL, NMSH, YHW, AES, and EN. Data were prepared and analyzed by JFGM under the supervision of JAS. JFGM drafted the manuscript with substantial feedback from JAS and subsequent critical edits from all other authors. All authors approved the final manuscript for submission.

\section{Competing Interest Statement}

None 


\begin{abstract}
Typologies organize knowledge and advance theory for many scientific disciplines, including more recently in psychological science. However, no typology exists to categorize use of emotion regulation strategies. This is surprising given emotion regulation skills are used daily and are robustly linked with mental health symptoms. We attempted to identify and validate a working typology of emotion regulation across six samples (Total $\mathrm{N}=1492$, from multiple populations) by using several computational techniques. We uncovered evidence for three types of regulators: an infrequently regulating type (Lo), a frequently regulating type (Hi), and a third type (Mix) that selectively titrates strategy usage. Individuals in the Mix type exhibited the most adaptive mental health symptoms. These differences were stable over time and across different samples. These results are important for basic understanding of emotion regulation and for informing future interventions aimed at improving mental health.
\end{abstract}

Keywords: Emotion regulation; Mental Health; Typology; Emotion 


\section{EMOTION REGULATION TYPES}

\section{A Data-Driven Typology of Emotion Regulation Profiles}

Scientific advancement often relies on the construction, validation, and subsequent use of typologies: comprehensive and generalizable systems of categorization based on relevant characteristics. Typological knowledge structures have been critical for shaping theories and sparking insights across a range of scientific disciplines, spanning fields such as chemistry, biology, archaeology, and linguistics (Greenberg, 1960; Hill \& Evans, 1972; Kaupp, 2007; Kluge \& Wolf, 1993). While it can be difficult to define psychological typologies (Costa Jr. et al., 2002; Donnellan \& Robins, 2010; Gardner \& Martinko, 1996), it can be done with appropriate computational and statistical methodologies (Gerlach et al., 2018; Lindström et al., 2021; Wray-Lake \& Shubert, 2019), and may allow people to be meaningfully categorized on the basis of psychological traits ranging from personality to social reward learning (Gerlach et al., 2018; Lindström et al., 2021; Wray-Lake \& Shubert, 2019). Somewhat surprisingly, however, there exist no data-driven typologies of emotion regulation, despite its central role in mental health as a transdiagnostic domain of impairment. Here, we use computational and advanced statistical tools in six samples to develop a typology of how individuals regulate emotion and to evaluate how it maps onto mental health symptoms.

Humans can use various strategies to regulate their emotions, and the particular strategies they select have implications for wellbeing and mental health (Gross, 2015). Gross' (1998) widely adopted process model of emotion and emotion regulation contends that emotions unfold in a series of stages (Gross, 2015). In this model, emotions are initiated when an individual is placed in a situation containing affectively evocative features. One may then pay attention to emotional stimuli and appraise, or 'make meaning' by assessing their motivational relevance (Moors et al., 2013). In turn, appraisals give rise to responses, behavioral and physiological manifestations of emotion. At each of these stages, there theoretically lies an opportunity for regulation in accordance with one's goals ${ }^{1}-$ be it by selecting one's

\footnotetext{
${ }^{1}$ Emotion regulation can also occur in an implicit, automatic fashion (Braunstein et al., 2017; Etkin et al., 2015), or as an incidental byproduct of other actions (e.g., putting one's feelings into words). We do not consider these types of regulation in this study.
} 


\section{EMOTION REGULATION TYPES}

situation (situation selection; Webb et al., 2018), modifying attention (selective attention or distraction; Critchley et al., 2000; Sheppes \& Meiran, 2008; Van Dillen et al., 2009), changing appraisals to alter emotional experience (cognitive reappraisal; John \& Gross, 2004), or controlling overt emotional responses such as facial expressions (expressive suppression; John \& Gross, 2004).

While these five strategies (situation selection, selective attention, distraction, reappraisal, suppression) do not exhaustively describe the human repertoire for emotion regulation, they are particularly important because they can be deployed intentionally and thus represent natural targets for interventions (Braunstein et al., 2017; Denny \& Ochsner, 2014). Indeed, a core element of many evidence-based interventions for both youth and adult psychiatric illness consists of bolstering skills such as reappraisal (Linehan, 2014; Milne \& Reiser, 2017). Despite the fact that emotion regulation can occur with a variety of strategies, prior empirical studies have largely focused on identifying mechanisms and consequences of individual strategies, rather than jointly examining many strategies. Most of this work has focused on the strategies reappraisal and suppression, in part due to their robust and divergent links with adjustment outcomes (Brockman et al., 2017; Haines et al., 2016; Webb et al., 2012). Seldom have prior studies measured more than one or two strategies in the same sample of participants (Chervonsky \& Hunt, 2019; Haines et al., 2016; Kelley et al., 2019), and the few studies that have assessed multiple strategies in the same individuals have focused on specialized research questions such as regulating food intake (Kelley et al., 2019; Lopez et al., 2021) as opposed to broader emotional phenomena. Thus, while current theories make predictions about how individuals use sets of strategies, empirical data is constrained to a far narrower subset of strategies.

Expanding the scope of emotion regulation research to include other strategies promises to shed light on important yet unanswered questions. One such question is whether individual differences in emotion regulation strategy usage across a set of strategies can be categorized into a typology. Since trait differences in emotion regulation usage ostensibly lie on continua, a typology in this context refers to distinct patterns of collective strategy usage among individuals. Thus, individuals' strategy usage is 


\section{EMOTION REGULATION TYPES}

represented by clusters in a five-dimensional space ( 1 dimension for each strategy) and types need not correspond to 'natural kinds' but must instead simply be characterized by sufficiently high within-cluster similarity (consistent with other recent typological attempts, Gerlach et al., 2018). At least two areas of existing research hint at the existence of a typology of emotion regulation strategy usage. First, theoretical frameworks posit patterns of individual differences in individual strategy usage (Gross \& John, 2003), which could indicate that individuals can be categorized into types on the basis of their collective strategy usage (e.g., one group could be individuals who use strategies 1-3 but not 4-5, whereas another group might show the opposite pattern). Second, psychological typologies have been identified in adjacent areas of research including social learning (Lindström et al., 2021), prosocial behavior (Wray-Lake \& Shubert, 2019), and self-regulatory flexibility (Chen \& Bonanno, 2021). Perhaps most relevant, a recent study showed that individual differences in core personality traits (e.g., Big Five) follow a meaningful typology (Gerlach et al., 2018). This finding has direct implications for the present study inasmuch as emotion regulation and personality tendencies may both be characterized as stable traits (Gross, 2015), and personality influences both bottom-up emotional processes and top down emotion regulation (Canli et al., 2001; Hughes et al., 2020; Pollock et al., 2016; Yoon et al., 2013).

In the current study we used data-driven methods to identify and validate a stable typology of emotion regulation strategy usage (Gerlach et al., 2018). We then characterized potential regulatory types by identifying clusters of regulatory patterns between individuals, and further how regulatory types within this typology are associated with two types of mental health symptoms: affective symptoms (transdiagnostic affective phenomena implicated in various diagnoses) and clinical symptoms (symptomatology closely related to specific clinical diagnoses). We concluded our investigation by examining strategy-level associations in the hopes of identifying potential mechanisms that could explain underlying differences in mental health symptoms between clusters.

\section{Methods}

\section{Methodological \& Analytic Overview}




\section{EMOTION REGULATION TYPES}

The present study was carried out using six independent datasets comprised in aggregate of 1492 individuals from various populations, underscoring the robustness and comprehensiveness of our approach. Data, materials, and code for this report are publicly available on the Open Science Framework (OSF; osf.io/d2hku). Comprehensive details on the following sections are provided in the Supplementary Information: Section 1 - Participants and Sampling Strategy; Section 2 - Materials; Section 3 Psychometric Validation of the E-ERQ; Section 4 - Analytic Procedure; Section 5 - Individual StrategySymptom Association Results). This section contains a summary of the materials and methods used in the current study.

\section{Materials.}

Measure creation and validation. The first step in the present research was to create and validate a self-report measure of emotion regulation strategy usage, termed the E-ERQ. The E-ERQ is comprised of novel items intended to assess the use of three understudied strategies (distraction, selective attention, and situation selection) with existing items from the original Emotion Regulation Questionnaire (Gross \& John, 2003) to assess two well-studied strategies (reappraisal and suppression). Novel items were validated in three of our independent samples using item response theory and factor analysis methods (detailed in Section 3 of the Supplementary Information), and were found to have good psychometric properties.

Assessing Mental Health Symptoms. To evaluate links between emotion regulation strategy usage and mental health, we assessed two types of mental health symptoms. First, we examined transdiagnostic “affective symptoms," which consisted of trait affect (measured with the PANAS, Watson et al., 1988)

and emotional expressivity (measured with the BEQ, ref 42). Second, we evaluated "clinical symptoms", comprised of measures that specifically assess depression and anxiety symptomatology as well as unspecified internalizing symptomatology (symptoms that are common across depression and anxiety). We focused on these symptoms because they are common in the general population (2017 National Survey on Drug Use and Health: Methodological Summary and Definitions, 2018; Lépine, 2002; Lim et 
al., 2018; Nelson et al., 2008; Wiegner et al., 2015), consequential for well-being (Bekhbat \& Neigh, 2018; Karling et al., 2016; Leonard, 2010; Nielsen et al., 2008; Penninx et al., 2013), and are frequently treated with psychotherapies that target emotion regulation skills (Covin et al., 2008; Durlak et al., 1991; Stewart \& Chambless, 2009). Anxious and depressive symptoms were measured with the Mini-MASQ (Clark \& Watson, 1995)²; nonspecific internalizing symptomatology was broken down into general distress (Mini-MASQ) and perceived stress (Cohen et al., 1983). Finally, longitudinal assessments of anxiety were examined longitudinally in LAB 2. Details regarding longitudinal data and information about other collected measures are included in the Supplementary Information (Section 2).

\section{Analytic Procedure}

Toward a Typology of Emotion Regulation Strategy Usage.

Establishing a Typology of Emotion Regulation via Clustering. In total, the E-ERQ was administered to six independent samples comprised of 1492 participants. Before this, the E-ERQ was validated in three online samples (ONLINE1, ONLINE2, ONLINE3). A sixth and final sample (TESTRETEST) was collected to assess the test-retest reliability of clustering results. Correlations between emotion regulation strategy scores are listed by sample in Supplementary Table 8.

Across the six samples, we used K-Medoid clustering as a data-driven method of searching for a prospective typology of emotion regulation strategy usage on the E-ERQ. In other words, we sought to group subjects into clusters based on how much they reported using the five evaluated emotion regulation strategies (situation selection, selective attention, distraction, reappraisal, suppression). This technique partitions sample observations into $k$ clusters by creating boundaries that minimize within cluster variance by selecting an exemplar point at the center of each cluster and evaluating each observation's distance to

\footnotetext{
2 One of our samples assessed depressive symptoms with the Center for Epidemiologic Studies Depression Scale (CES-D) and anxiety symptoms with the 12-item generalized anxiety subscale Screen for Adult Anxiety Related Disorders (SCAARED). This sample did not have measurements of affective symptoms available.
} 


\section{EMOTION REGULATION TYPES}

said exemplar. In K-Medoids clustering, the exemplary points are actual observations in the dataset that can be thought of as a geometric median, and as such are robust to noise. We tested 5 candidate $k$ values (cluster numbers), 2-6. More information about our rationale for using and assumptions about K-

Medoids, in addition to descriptions of several robustness checks (including sensitivity analyses and testretest reliability), is included in the Supplementary Information (Section 4).

Differences in Regulatory Strategy Usage by Cluster. After obtaining a reliable cluster solution, we sought to unpack differences in regulatory strategy usage between clusters by tabling and computing means for each regulatory strategy per cluster across studies. Importantly, we did not perform inferential statistics on cluster differences between each regulatory strategy to avoid circular inference (clusters are already optimized to be maximally distinct). Cluster means for each strategy, across the five main samples (ONLINE1-3, LAB1-2) are listed in Supplementary Table 9.

Differences in Mental Health Symptoms by Cluster. We next probed differences in mental health symptoms by cluster ${ }^{3}$ in the five samples for which we had assessed links between the E-ERQ and mental health symptoms. This was done by computing pairwise Cohen's $d$ values between clusters for all mental health symptoms across all samples that had such data available (4-5 samples, depending on trait/mental health outcome). Analytic 95\% confidence intervals (CIs) were calculated to infer meaningful differences between clusters. For ease of interpretability, and because results of this type were generally consistent across samples, Cohen's $d$ values and accompanying CIs from each sample for a given metric were averaged to give an 'overall' estimate of cluster differences across all samples and are reported in Supplementary Tables 10 (affective symptoms) and 12 (clinical symptoms). Sample-specific results are also reported in the Supplementary Information (Supplementary Tables 11 and 13). In addition to these cross-sectional analyses, we were also able to conduct longitudinal analyses on the one sample (LAB2) that assessed anxiety over time after administration of the E-ERQ. To do so, we used longitudinal growth

\footnotetext{
${ }^{3}$ Though we find evidence for different regulatory types, we opt to use the term 'cluster' or 'cluster membership' throughout to describe individuals corresponding to a given type in order to avoid giving a premature impression of objectivity or finality about these types.
} 


\section{EMOTION REGULATION TYPES}

modeling in a multilevel framework to predict changes in anxiety as a function of cluster membership over a sixty-day period in LAB2 participants (full modeling details are in the Supplementary Information, Section 4).

Quantifying links between emotion regulation strategy usage and mental health symptoms.

\section{Examining Associations between Strategies, Affective Symptoms, and Mental Health Symptoms} with Ridge Regression. After examining between-cluster differences in mental health symptoms, we turned our attention to uncovering potential mechanisms that could be driving identified differences. We accomplished this by using ridge regression to regress each affective and mental health symptom onto participants' mean scores on each of the five E-ERQ emotion regulation strategies (while statistically adjusting for all strategies), allowing us to understand relationships between trait-level usage of each emotion regulation strategy and a given affective or clinical symptom. For brevity and ease of interpretability, and because results of this type were generally consistent across samples, parameter estimates and accompanying CIs from each sample for a given metric were averaged to give an 'overall' estimate of strategy-affective symptoms (Supplementary Figure 4) or strategy-clinical symptoms association (Supplementary Figure 5). However, we encourage readers to consult figures and tables in the Supplementary Information to appreciate sample-to-sample heterogeneity. Ridge regression details are also included in the Supplementary Information (Section 4).

\section{Using Cross-Validation and Growth Modeling to Quantify the Predictive Utility of Strategies. We} used a combination of regularized regression and cross-validation to investigate whether we could predict mental health symptoms in a new dataset based on emotion regulation strategy usage from a trained dataset. We first quantified associations between a given strategy and a given mental health symptom (while statistically adjusting for other strategies) across various samples. We next examined the predictive utility of these strategies as a set, by leveraging our multiple independent samples and subjecting them to cross-validation. For each affective and clinical symptom, we fit a ridge regression model using pooled data from $s-1$ samples, used the ensuing regression coefficients to predict scores on the symptom in 


\section{EMOTION REGULATION TYPES}

sample $s$, and computed $R^{2}$ as our metric of prediction accuracy. This procedure was repeated until each independent sample had served as the hold out sample (i.e., there were 5 iterations of this procedure) $R^{2}$ statistics for each held out sample were averaged and error bars were derived from the sample-to-sample variability of the statistics.

In a second set of analyses, we leveraged growth curve modeling with LAB2's longitudinal data to examine how inter-individual differences in strategy usage (adjusting for all other strategies) prospectively predicted anxiety (loneliness was also assessed and will be reported in a forthcoming manuscript). This analysis was performed to gauge temporal stability of associations between strategies and to identify potential causal mechanisms. While longitudinal data do not guarantee causality, their temporal component is an important component that provides valuable information about causal processes, while also providing information on prospective associations involving strategy usage. To this end, the five strategies were entered as predictors, along with other key covariates, in a growth curve model predicting anxiety over a sixty-day period. Full growth curve modeling details are in the Supplementary Information (Section 4).

\section{Results}

Aims and participants. Across six independent samples totaling 1492 participants (ONLINE1, ONLINE2, ONLINE3, LAB1, LAB2, TEST-RETEST; sample characteristics reported in Table 1), this study addressed three overarching goals: i) to identify evidence for a typology of individual differences in emotion regulation strategy usage using data-driven clustering methods and unpack differences in regulatory strategy usage between potential typological clusters; (ii) to understand the consequences of cluster membership by quantifying relationships between typology cluster and a constellation of mental health symptoms (divided into two categories: affective symptoms and clinical symptoms); (iii) to uncover potential mechanisms behind how and why there exist differences among clusters by examining strategy-level associations with mental health symptoms using ridge regression, cross-validation, and growth curve modeling. 


\section{EMOTION REGULATION TYPES}

\section{Clustering Algorithms Suggest a Typology of Emotion Regulation Strategy Usage}

Identifying the Number of Clusters. To achieve the aforementioned goals, we created and validated a measure termed the Extended Emotion Regulation Questionnaire (E-ERQ, items listed in Supplementary Table 1), which assesses the frequency with which individuals used five emotion regulation strategies (reappraisal, expressive suppression, distraction, selective attention and situation selection; see Section 3 of Supplementary Information for measure development and validation details). We next examined whether individuals "clustered" into different types in how they reported using emotion regulation strategies on this measure. In looking across the 6 samples, three clusters $(k=3)$ emerged as the optimal solution in each sample. Elbow plots of within cluster sum of squares always indicated $k=3$ and gap statistics indicated $k=3$ in all but one sample (LAB2). Robustness checks with tSNE-reduced data always converged on $k=3$. Re-running the analysis with a more parsimonious cluster solution $(k=2)$ did not yield a replicable pattern of strategy usage across samples, further enhancing confidence in the $k=3$ solution (Full details of all robustness checks are located in Section 4 of the Supplementary Material). The three clusters represented relatively event portions of individuals in each sample.

Examining Patterns of Regulatory Strategy Usage among Clusters. Once we obtained a cluster solution, we used descriptive statistics to characterize how emotion regulation strategy usage differed between clusters (Figure 1; Supplementary Table 9). Individuals in one cluster reported consistently high usage of emotion regulation, regardless of strategy ('Hi'). By contrast, individuals in a second cluster reported uniformly low emotion regulation strategy usage ('Lo'). A third cluster of individuals reported a selective, mixed pattern of strategy usage ('Mix'). Mix cluster individuals consistently endorsed high reappraisal and situation selection usage at levels comparable with the Hi cluster, and low suppression usage at levels comparable to the Lo cluster. These individuals also generally endorsed high distraction usage and low selective attention usage (again comparable with the Hi and Lo clusters, respectively), particularly in the online samples. The sample-to-sample consistency of these findings, coupled with 


\section{EMOTION REGULATION TYPES}

results from robustness checks (see Supplementary Information), suggest a reliable typology of regulatory strategy usage.

Cluster Stability. Cluster membership was observed to be quite stable both between and within samples. Between samples, $81.77 \%$ of participants were assigned to the same cluster in both their study sample and an aggregated sample. This estimate was relatively consistent across samples (ONLINE1 = $83.04 \%$, ONLINE2 $=87.44 \%$, ONLINE3 $=75.08 \%$, LAB $1=80.31 \%$, LAB2 $=75.25 \%)$. To assess within-sample stability, we examined cluster membership over time in the TEST-RETEST dataset. $75.16 \%$ of individuals in the TEST-RETEST sample were assigned to the same clusters two weeks apart. Cluster stability exceeded what would be expected at chance, based on bootstrapping and simulation techniques (see Supplementary Information, Section 4).

\section{Cluster Membership is Associated with Differential Patterns of Mental Health Symptoms}

Probing Differences in Mental Health Symptoms by Cluster. Notably, the Mix cluster evinced the highest levels of trait positive affect, the lowest levels of trait negative affect, as well as the highest levels of both positive and negative emotional expressivity. The Lo cluster displayed low trait positive affect, high trait negative affect, and relatively high emotional expressivity. The Hi cluster had relatively high trait positive affect and low negative affect, and lowest level of both facets of emotional expressivity. Generally this suggests that individuals in the Mix group experience more positive and less negative affect and strongly express their emotions, whereas individuals in the Hi group appear to have slightly less positive emotional experiences and are markedly less expressive. Individuals in the low group have the most negative emotional experiences, but are also high in expressivity. Raincloud charts (Row 1) and effect size plots (Row 2) in Figure 2 depict how affective symptoms differed between the three clusters (aggregated across samples); Supplementary Tables 10-11 list pairwise effect sizes and 95\% confidence intervals between clusters (averaged across samples, broken down by samples). Raincloud charts by sample are depicted in Supplementary Figure 7. 


\section{EMOTION REGULATION TYPES}

Individuals in the Mix cluster showed strong mental health across all measures - scoring the lowest on anxiety, depression, general distress, and perceived stress. By contrast, individuals in the Lo cluster showed poor mental health - generally scoring the highest on anxiety, depression, general distress, and perceived stress. Individuals in the Hi cluster showed an intermediate phenotype, such that they tended to report better mental health than the Lo cluster but worse mental health than the Mix cluster. Somewhat surprisingly, their scores were more comparable to the Lo group than the Mix cluster on anxiety and depression. Between-cluster differences in clinical symptoms are shown in Figure 3 while Supplementary Tables 12-13 lists average pairwise effect sizes and 95\% confidence intervals between clusters (averaged across samples, broken down by samples). Raincloud charts by sample are depicted in Supplementary Figure 8. Growth modeling results (Supplementary Figure 11; Supplementary Table 18) revealed that associations between cluster membership and mental health are stable across time, as cluster differences in anxiety were preserved over a sixty-day span (growth trajectories did not differ between cluster; see Supplementary Information). Overall, these results suggest that cluster membership is a meaningful indicator of mental health.

\section{Strategy-Level Associations Reveal Potential Mechanisms behind Cluster Differences}

Using ridge regression, we generally found that greater reappraisal usage was related to more adaptive patterns of affective and clinical symptoms; greater suppression usage was related to more maladaptive patterns of affective and clinical symptoms. We also observed that distraction was associated with more maladaptive affective and clinical symptoms, and situation selection was associated with more adaptive affective symptoms but was not associated with clinical symptoms. Selective attention was not strongly associated with any symptoms. Aggregated results are shown in Supplementary Figures 4 and 5. A more detailed description of these results can be accessed in the Supplementary Information (Section 5).

\section{Assessing the Predictive Utility of all Strategies.}




\section{EMOTION REGULATION TYPES}

Out of Sample Prediction. Next, we examined how accurately emotion regulation strategy usage predicted mental health symptoms in novel samples (Supplementary Figure 6). This was done because understanding how well the strategies collectively predict variance in affective symptoms and clinical symptoms can help explain how cluster differences emerge. Doing so in a cross-validation framework provides the additional benefit of understanding how predictive the set of strategies are in novel samples, a necessity for translating this knowledge to applied settings. In terms of affective symptoms, strategy usage was most strongly associated with the expressivity variables $\left(R^{2}=0.362-0.396\right)$, followed by trait affect $\left(R^{2}=0.115-0.197\right)$. In terms of clinical symptoms, depression $\left(R^{2}=0.222\right)$ and perceived stress $\left(R^{2}=0.213\right)$ were most strongly influenced by the set of strategies, followed by general distress $\left(R^{2}=0.157\right.$; though the predictive accuracy of general distress varied more widely from sample to sample). Effect sizes ranged between the medium and large benchmarks. By contrast, anxiety was only modestly related to the set of strategies as a whole $\left(R^{2}=0.063\right)$.

Longitudinal Prediction. Results from growth curve modeling revealed that cluster membership and strategy usage predicted differences in anxiety, and that these differences were highly stable over a sixty-day period. Growth curve modeling methods and statistics (Supplementary Tables 18-19) are reported in the Supplementary Information.

\section{Discussion}

Using a series of computational tools in six samples, we identified a typology of emotion regulation strategy usage such that one cluster of individuals was likely to use all strategies frequently (Hi), a second cluster was likely to use all strategies infrequently (Lo), and a third cluster selectively used some, but not all, strategies (Mix). We then observed that individuals in the Mix cluster tended to report better mental health. Lastly, we uncovered potential mechanisms behind these cluster differences, suggesting increased reliance on cognitive reappraisal and situation selection in conjunction with decreased reliance on suppression and distraction seems to be associated with optimal mental health. These findings have ramifications that stretch across multiple scientific domains. 


\section{EMOTION REGULATION TYPES}

Implications for Theories of Human Emotion. Our findings inform models of emotion regulation in several ways. For instance, that specific strategies tended to cluster together implies that distinct emotion regulation strategies (e.g., reappraisal and distraction) may actually share core psychological features. If true, this raises interesting questions about whether emotion regulation strategies are best conceptualized as occupying discrete categories or as collections of response tendencies that exist on continua (Ford et al., 2017; Gross \& Feldman Barrett, 2011). An alternative interpretation is individuals may frequently use a given pair of strategies because said individuals are adept at selecting effective strategies and not because the pair of strategies share common mechanism (Chen \& Bonanno, 2021). With that said, it is also possible that unobserved moderating variables (e.g., personality, executive functions, etc.) also help explain cluster differences. Rigorously testing these possible interpretations will be critical for subsequent work.

Our cluster-level findings highlight the importance of assessing multiple emotion regulation strategies in a given sample while also replicating established strategy-symptom associations (e.g., that expressive suppression is linked to maladjustment, Webb et al., 2012). Our results also show that strategies have additive effects - that is, their predictive utility appears to build when an individual uses them in concert (Lopez et al., 2021). This is notable because many studies of emotion regulation assume individuals often specialize in a single strategy (Gross \& John, 2003), whereas theories of emotion regulation posit that using many strategies has cumulative advantages (Aldao et al., 2016). Crucially, our results raise the question of how individuals use multiple strategies. That is, do individuals tend to combine or use strategies in rapid succession in a single instance, or do they flexibly toggle between strategies according to situation-specific demands? These questions complement a burgeoning literature

on emotion regulation flexibility, — how well individuals flexibly use different strategies based on metacognitive skills (Chen \& Bonanno, 2021) — as well as polyregulation — when individuals use more than one emotion regulation strategy concurrently or sequentially within an emotional episode (Ford et al., 2019). Future work could investigate these competing possibilities using ecological momentary 


\section{EMOTION REGULATION TYPES}

assessment methodologies (Larson et al., 1980; Larson \& Lampman-Petraitis, 1989) or with creative laboratory designs that involve high density sampling of self-reported affective experiences in response to dynamic stimuli (Zaki et al., 2008).

Applications in Clinical Science. We found that regulatory "type" (i.e., clusters) membership correlated with mental health symptoms. Specifically, individuals in the Mix group tended to exhibit more adaptive mental health profiles than individuals in the Hi or Lo regulation groups. These findings indicate that regulatory "types" (i.e., clusters) may be an important transdiagnostic predictor of emotional health, especially given that cluster membership showed high out-of-sample and longitudinal (withinperson) predictive validity. Such knowledge could inform precision medicine and personalized therapeutic approaches (Doré et al., 2016; Gratton et al., 2018; Robinson, 2012). For example, knowing whether an individual is already using a given set of regulation strategies can help clinicians plan effective courses of treatment. Alternately, this brief questionnaire could be used help screen individuals in appropriate contexts, such as incoming college freshman who are especially susceptible to internalizing psychopathology (Guassi Moreira \& Telzer, 2015).

Relevance for Trait Typologies in Psychology. Our results lend credence to a growing interest in identifying psychological typologies. Our evidence for a reliable typology lends confidence to recent typological efforts (Chen \& Bonanno, 2021; Lindström et al., 2021), and also raises the question of whether individual trait-level typologies are perhaps nested in a broader unifying framework of psychological traits. For example, given recent work by Gerlach and Colleagues (2018) identifying a typology of personality traits, and the fact that personality traits and emotion regulation are intrinsically linked (Saucier \& Iurino, 2019), it is possible that our typology is nested within the one identified by Gerlach and colleagues or another one altogether. Future work could test this possibility by mapping personality traits to emotion regulation strategies, in addition to examining relationships between prospective personality types and our prospective emotion regulation types. 


\section{EMOTION REGULATION TYPES}

Limitations and Future Directions. Several important limitations apply to this study. We do not know at present whether this typology relates to individuals' ability to use different regulatory strategies (Silvers \& Guassi Moreira, 2019), or their beliefs about emotion regulation (Ford \& Gross, 2019). Future work would benefit from multi-modal assessments of emotion regulation skills (e.g., self-report, otherreport, laboratory task, physiological assessments, etc.). Relatedly, running confirmation studies in larger samples would offer possibilities to use more sophisticated clustering algorithms, such as Gaussian mixture modeling which can assign membership probabilistically (Gerlach et al., 2018). More comprehensive test-retest designs could also be added to these endeavors (e.g., testing at 4, 6, or 8 week intervals), as we assessed test-retest reliability with a two week interval. Finally, to better understand the ontogeny of the prospective regulation types, longitudinal developmental work is needed to determine when and how prospective types are formed, their stability, their relationship to temperament (Morales \& Fox, 2019). Such work would also help uncover the direction of potential causal arrows in associations between cluster membership and mental health.

Acknowledgments. Preparation of this manuscript was supported by a National Science Foundation Graduate Research Fellowship (2016220797) and a National Institutes of Health Predoctoral T32 Fellowship to JFGM, and generous funds from the UCLA department of psychology to JAS. We are grateful for reflections on the study concept from members of the Social Affective Neuroscience and Development (PI: Jennifer Silvers, PhD), Computational Social Neuroscience (PI: Carolyn Parkinson, 


\section{EMOTION REGULATION TYPES}

$\mathrm{PhD}$ ), and Quantitative Research Collaboratory (PI: Amanda Montoya, PhD) Laboratories at UCLA, in addition to feedback from Drs. Bruce Doré and Steven Reise. 


\section{References}

2017 National survey on drug use and health: Methodological summary and definitions. (2018). Substance Abuse and Mental Health Services Administration.

Aldao, A., Gee, D. G., De Los Reyes, A., \& Seager, I. (2016). Emotion regulation as a transdiagnostic factor in the development of internalizing and externalizing psychopathology: Current and future directions. Development and Psychopathology, 28(4pt1), 927-946. https://doi.org/10.1017/S0954579416000638

Altman, N., \& Krzywinski, M. (2017). Points of significance: Clustering. Nature Methods, 14(6), 545546.

Bekhbat, M., \& Neigh, G. N. (2018). Sex differences in the neuro-immune consequences of stress: Focus on depression and anxiety. Brain, Behavior, and Immunity, 67, 1-12.

Bornovalova, M. A., Choate, A. M., Fatimah, H., Petersen, K. J., \& Wiernik, B. M. (2020). Appropriate use of bifactor analysis in psychopathology research: Appreciating benefits and limitations. Biological Psychiatry, 88(1), 18-27.

Brandao, T., Schulz, M. S., Gross, J. J., \& Mena Matos, P. (2017). The emotion regulation questionnaire in women with cancer: A psychometric evaluation and an item response theory analysis. PsychoOncology, 26, 1647-1653.

Braunstein, L. M., Gross, J. J., \& Ochsner, K. N. (2017). Explicit and implicit emotion regulation: A multi-level framework. Social Cognitive and Affective Neuroscience, ePub ahead, 1-13. https://doi.org/10.1080/02699931.2010.544160

Brockman, R., Ciarrochi, J., Parker, P., \& Kashdan, T. (2017). Emotion regulation strategies in daily life: Mindfulness, cognitive reappraisal and emotion supression. Cognitive Behaviour Therapy, 46(2), 91-113.

Calderon Leon, M., Guassi Moreira, J. F., Saragosa-Harris, N., Waizman, Y., Sedykin, A., Peris, T. S., \& Silvers, J. A. (2020). Parent and friend relationship quality and trajectories of loneliness during the first year of college. PsyArXiv, 1-43.

Canli, T., Zhao, Z., Desmond, J. E., Eunjoo, K., Gross, J. J., \& Gabrieli, J. D. E. (2001). An fMRI study of personality influences on brain reactivity to emotional stimuli. Behavioral Neuroscience, 115(1), $33-42$.

Chalmers, R. P. (2012). mirt: A multidimensional item response theory package for the R environment. Journal of Statistical Software, 48(6), 1-29.

Chen, S., \& Bonanno, G. A. (2021). Components of emotion regulation flexibility: Linking latent profiles to depressive and anxious symptoms. Clinical Psychological Science, in press, 1-16.

Chervonsky, E., \& Hunt, C. (2019). Emotion regulation, mental health, and social wellbeing in a young adolescent sample: A concurrent and longitudinal investigation. Emotion, 19(2), 270-282.

Clark, L. A., \& Watson, D. (1991). Tripartite model of anxiety and depression: Psychometric evidence and taxonomic implications. Journal of Abnormal Psychology, 100(3), 316.

Clark, L. A., \& Watson, D. (1995). The mini mood and anxiety symptom questionnaire (Mini-MASQ). Unpublished Manuscript, University of Iowa.

Cohen, S., Kamarck, T., \& Mermelstein, R. (1983). A global measure of perceived stress. Journal of 


\section{EMOTION REGULATION TYPES}

Health and Social Behavior, 24(4), 385-396. https://doi.org/10.2307/2136404

Costa Jr., P. T., Herbst, J. H., McCrae, R. R., Samuels, J., \& Ozer, D. L. (2002). The replicability and utility of three personality types. European Journal of Personality, S73-S87.

Covin, R., Ouimet, A. J., Seeds, P. M., \& Dozois, D. J. A. (2008). A meta-analysis of CBT for pathological worry among clients with GAD. Journal of Anxiety Disorders, 22(1), 108-116. https://doi.org/10.1016/j.janxdis.2007.01.002

Critchley, H., Daly, E., Phillips, M., Brammer, M. J., Bullmore, E. T., Williams, S. C. R., Van Amelsvoort, T., Robertson, D., David, A., \& Murphy, D. G. (2000). Explicit and implicit neural mechanisms for processing of social information from facial expressions: A functional magnetic resonance imaging study. Human Brain Mapping, 9(2), 93-105.

Dalmaijer, E. S., Nord, C. L., \& Astle, D. E. (2020). Statistical power for cluster analysis. ArXiv.

Denny, B. T., \& Ochsner, K. N. (2014). Behavioral effects of longitudinal training in cognitive reappraisal. Emotion, 14(2), 425-433. https://doi.org/10.1037/a0035276.Behavioral

Donnellan, M. B., \& Robins, R. W. (2010). Resilient, overcontrolled, and undercontrolled personality types: Issues and controversies. Social and Personality Psychology Compass, 4(11), 1070-1083.

Doré, B. P., Silvers, J. A., \& Ochsner, K. N. (2016). Toward a Personalized Science of Emotion Regulation. Social and Personality Psychology Compass, 10(4), 171-187.

Durlak, J. A., Fuhrman, T., \& Lampman, C. (1991). Effectiveness of cognitive-behavior therapy for maladapting children: a meta-analysis. Psychological Bulletin, 110(2), 204-214. https://doi.org/10.1037/0033-2909.110.2.204

Etkin, A., Büchel, C., \& Gross, J. J. (2015). The neural bases of emotion regulation. Nature Reviews Neuroscience, 16(11), 693-700. https://doi.org/10.1038/nrn4044

Ford, B. Q., \& Gross, J. J. (2019). Why beliefs about emotion matter: An emotion-regulation perspective. Current Directions in Psychological Science, 28(1), 74-81.

Ford, B. Q., Gross, J. J., \& Gruber, J. (2019). Broadening our field of view: The role of emotion polyregulation. Emotion Review, 11(3), 197-208. https://doi.org/10.1177/1754073919850314

Ford, B. Q., Karnilowicz, H. R., \& Mauss, I. B. (2017). Understanding reappraisal as a multicomponent process: The psychological health benefits of attempting to use reappraisal depend on reappraisal success. Emotion, 17(6), 905-911. https://doi.org/10.1037/emo0000310

Gardner, W. L., \& Martinko, M. J. (1996). Using the Myers-Briggs Type Indicator to study managers: A literature review and research agenda. Journal of Management, 22(1), 45-83.

Gerlach, M., Farb, B., W., R., \& Amaral, L. A. N. (2018). A robust data-driven approach identifies four personality types across four large data sets. Nature Human Behaviour, 2, 735-742.

Gratton, C., Laumann, T. O., Nielsen, A. N., Greene, D. J., Gordon, E. M., Gilmore, A. W., Nelson, S. M., Coalson, R. S., Snyder, A. Z., Schlaggar, B. L., Dosenbach, N. U. F., \& Petersen, S. E. (2018). Functional brain networks are dominated by stable group and individual factors, not cognitive or daily variation. Neuron, 98(2), 439-452.e5. https://doi.org/10.1016/j.neuron.2018.03.035

Greenberg, J. H. (1960). A quantitative approach to the morphological typology of language. International Journal of American Linguistics, 26(3), 178-194.

Gross, J. J. (1998). The emerging field of emotion regulation: an integrative review. Review of General 


\section{EMOTION REGULATION TYPES}

Psychology, 2(5), 271-299. https://doi.org/10.1017.S0048577201393198

Gross, J. J. (2015). Emotion regulation: Current status and future prospects. Psychological Inquiry, 26(1), 1-26. https://doi.org/10.1080/1047840X.2014.940781

Gross, J. J., \& Feldman Barrett, L. (2011). Emotion generation and emotion regulation: One or two depends on your point of view. Emotion Review, 3(1), 8-16. https://doi.org/10.1177/1754073910380974

Gross, J. J., \& John, O. P. (1997). Revealing feelings: Facets of emotional expressivity in self-reports, peer ratings, and behavior. Journal of Personality and Social Psychology, 72, 435-448.

Gross, J. J., \& John, O. P. (2003). Individual differences in two emotion regulation processes: implications for affect, relationships, and well-being. Journal of Personality and Social Psychology, 85(2), 348-362. https://doi.org/10.1037/0022-3514.85.2.348

Guassi Moreira, J. F., \& Telzer, E. H. (2015). Changes in family cohesion and links to depression during the college transition. Journal of Adolescence, 43, 72-82. https://doi.org/10.1016/j.adolescence.2015.05.012

Haines, S. J., Gleeson, J., Kuppens, P., Hollenstein, T., Ciarrochi, J., Labuschagne, I., Grace, C., \& Koval, P. (2016). The wisdom to know the difference: Strategy-situation fit in emotion regulation in daily life is associated with well-being. Psychological Science, 27(12), 1651-1659. https://doi.org/10.1177/0956797616669086

Hill, J. N., \& Evans, R. K. (1972). A model for classification and typology. In D. L. Clarke (Ed.), Models in Archaeology (pp. 231-275). Metheun \& Co.

Hughes, D. J., Kratsiotis, I. K., Niven, K., \& Holman, D. (2020). Personality traits and emotion regulation: A targeted review and recommendations. Emotion, 20(1), 63-67.

Ireland, M. J., Goh, H. E., \& Marais, I. (2018). A Rasch model analysis of the emotion regulation questionnaire. Journal of Applied Measurement, 19(3), 258-270.

John, O. P., \& Gross, J. J. (2004). Healthy and unhealthy emotion regulation: Personality processes, individual differences, and life span development. Journal of Personality, 72(6), 1301-1333. https://doi.org/10.1111/j.1467-6494.2004.00298.x

Karling, P., Wikgren, M., Adolfsson, R., \& Norrback, K.-F. (2016). Hypothalamus-pituitary-adrenal axis hypersuppression is associated with gastrointestinal symptoms in major depression. Journal of Neurogastroenterology and Motility, 22(2), 292-303.

Kaupp, M. (2007). The role of radial nodes of atomic orbitals for chemical bonding and the periodic table. Journal of Computational Chemistry, 28(1), 320-325.

Kelley, N. J., Glazer, J. E., Pornpattananangkul, N., \& Nusslock, R. (2019). Reappraisal and suppression of emotion-regulation tendencies differentially predict reward-responsivity and psychological wellbeing. Biological Psychology, 140, 35-47.

Kluge, A. G., \& Wolf, A. J. (1993). Cladistics: What's in a word? Cladistics, 9(2), 183-199.

Larson, R., Csikszentmihalyi, M., \& Graef, R. (1980). Mood variability and the psycho-social adjustment of adolescents. Journal of Youth \& Adolescence, 9(6), 469-490. https://doi.org/10.1007/978-94-0179094-9_15

Larson, R., \& Lampman-Petraitis, C. (1989). Daily emotional states as reported by children and 


\section{EMOTION REGULATION TYPES}

adolescents. Child Development, 60(5), 1250-1260.

Leonard, B. E. (2010). The concept of depression as a dysfunction of the immune system. Current Immunology Reviews, 6(3), 205-212.

Lépine, J. P. (2002). The epidemiology of anxiety disorders: Prevalence and societal costs. The Journal of Clinical Psychiatry, 63, 4-8.

Lim, G. Y., Tam, W. W., Lu, Y., Ho, C. S., Zhang, M. W., \& Ho, R. C. (2018). Prevalence of depression in the community from 30 countries between 1994 and 2014. Scientific Reports, 8(2861).

Lindström, B., Bellander, M., Schultner, D. T., Chang, A., Tobler, P. N., \& Amodio, D. M. (2021). media engagement. Nature Communications, 1-10.

Linehan, M. (2014). DBT? Skills training manual (Second). Guilford Press.

Lopez, R., Cosme, D., Werner, K., Saunders, B., \& Hofmann, W. (2021). Associations between use of self-regulatory strategies and daily eating patterns: An experience sampling study in college-aged female. PsyArXiv.

McRae, K. (2013). Emotion regulation frequency and success: Separating constructs from methods and time scale. Social and Personality Psychology Compass, 7(5), 289-302.

Milne, D. L., \& Reiser, R. P. (2017). A manual for evidence-based CBT supervision (First). John Wiley \& Sons.

Moors, A., Ellsworth, P. C., Scherer, K. R., \& Frijda, N. H. (2013). Appraisal theories of emotion: State of the art and future development. Emotion Review, 5(2), 119-124.

Morales, S., \& Fox, N. A. (2019). A neuroscience perspective on emotional development. In V. LoBue, P.-E. K., \& K. Buss (Eds.), Handbook of Emotional Development (pp. 57-81). Springer, Cham.

Nelson, M. C., Story, M., Larson, N. I., Neumark-Sztainer, D., \& Lytle, L. A. (2008). Emerging adulthood and college-aged youth: An overlooked age for weight-related behavior change. Obesity, 16(10), 2205-2211. https://doi.org/10.1038/oby.2008.365

Nielsen, N. R., Kristensen, T. S., Schnohr, P., \& Grønbæk, M. (2008). Perceived stress and cause-specific mortality among men and women: Results from a prospective cohort study. American Journal of Epidemiology, 168(5), 481-491. https://doi.org/10.1093/aje/kwn157

Penninx, B. W. J. H., Milaneschi, Y., Lamers, F., \& Vogelzangs, N. (2013). Understanding the somatic consequences of depression: Biological mechanisms and the role of depression symptom profile. BMC Medicine, 11 .

Pollock, N. C., McCabe, G. A., Southard, A. C., \& Zeigler-Hill, V. (2016). Pathological personality traits and emotion regulation difficulties. Personality and Individual Differences, 95, 168-177.

Preece, D. A., Becerra, R., Hasking, P., McEvoy, P. M., Boyes, M., Sauer-Zavala, S., Chen, W., \& Gross, J. J. (2021). The emotion regulation questionnaire: Psychometric properties and relations with affective symptoms in a united states general community sample. Journal of Affective Disorders, in press.

Reise, S. P., Ainsworth, A. T., \& Haviland, M. G. (2005). Item response theory: Fundamentals, applications, and promise in psychological research. Current Directions in Psychological Science, 14(2), 95-101.

Reise, S. P., \& Waller, N. G. (2009). Item response theory and clinical measurement. Annual Review of 


\section{EMOTION REGULATION TYPES}

Clinical Psychology, 5, 27-48.

Robinson, P. N. (2012). Deep phenotyping for precision medicine. Human Mutation, 33(5), 777-780. https://doi.org/10.1002/humu.22080

Sahi, R. S., Ninova, E., \& Silvers, J. A. (2020). With a little help from my friends: Selective social potentiation of emotion regulation. Journal of Experimental Psychology: General.

Saucier, G., \& Iurino, K. (2019). High-dimensionality personality structure in the natural language: Further analyses of classic sets of english-language trait-adjectives. Journal of Personality and Social Psychology.

Sheppes, G., \& Meiran, N. (2008). Divergent cognitive costs for online forms of reappraisal and distraction. Emotion, 8(6), 870-874. https://doi.org/10.1037/a0013711

Silvers, J. A., \& Guassi Moreira, J. F. (2019). Capacity and tendency: A neuroscientific framework for the study of emotion regulation. Neuroscience Letters, 693, 35-39. https://doi.org/10.1016/j.neulet.2017.09.017

Stewart, R. E., \& Chambless, D. L. (2009). Cognitive-behavioral therapy for adult anxiety disorders in clinical practice: a meta-analysis of effectiveness studies. Journal of Consulting and Clinical Psychology, 77(4), 595-606. https://doi.org/10.1037/a0016032

Tibshirani, R., Walther, G., \& Hastie, T. (2001). Estimating the number of clusters in a data set via the gap statistic. Journal of the Royal Statistical Society: Series B, 63(2), 411-423.

Van Dillen, L. F., Heslenfeld, D. J., \& Koole, S. L. (2009). Tuning down the emotional brain: An fMRI study of the effects of cognitive load on the processing of affective images. NeuroImage2, 45(4), $1212-1219$.

Watson, D., Clark, L. A., \& Tellegen, A. (1988). Development and validation of brief measures of positive and negative affect: The PANAS scales. Journal of Personality and Social Psychology, 54(6), 1063-1070.

Webb, T. L., Lindquist, K. A., Jones, K., Avishai, A., \& Sheeran, P. (2018). Situation selection is a particularly effective emotion regulation strategy for people who need help regulating their emotions. Cognition \& Emotion, 32(2), 231-248.

Webb, T. L., Miles, E., \& Sheeran, P. (2012). Dealing with feeling: A meta-analysis of the effectiveness of strategies derived from the process model of emotion regulation. Psychological Bulletin, 138(4), 775-808. https://doi.org/10.1037/a0027600

Wiegner, L., Hange, D., Björkelund, C., \& Ahlborg, G. (2015). Prevalence of perceived stress and associations to symptoms of exhaustion, depression and anxiety in a working age population seeking primary care--an observational study. BMC Family Practice, 16(38), 1-8. https://doi.org/10.1186/s12875-015-0252-7

Wray-Lake, L., \& Shubert, J. (2019). Understanding stability and change in civic engagement across adolescence: A typology approach. Developmental Psychology, 55(10), 2169-2180.

Yoon, K. L., Maltby, J., \& Joormann, J. (2013). A pathway from neuroticism to depression: Examining the role of emotion regulation. Anxiety, Stress \& Coping, 26(5), 558-572.

Zaki, J., Bolger, N., \& Ochsner, K. (2008). It takes two: The interpersonal nature of empathic accuracy. Psychological Science, 19(4), 399-404. https://doi.org/10.1111/j.1467-9280.2008.02099.x 
24 EMOTION REGULATION TYPES 
Figure 2. Aggregate group differences between clusters on mental health symptoms (affective symptoms)
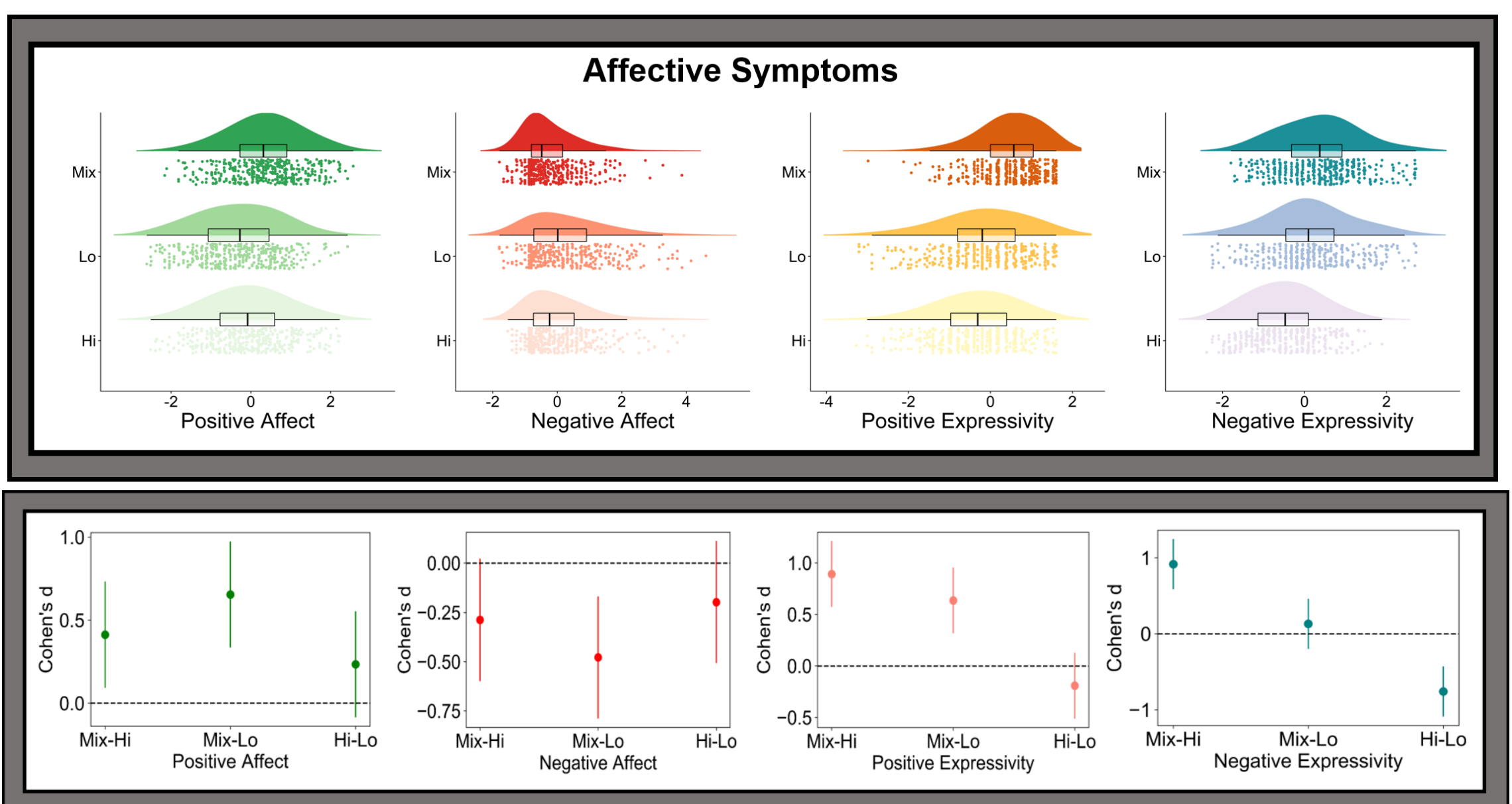

Note. 'Positive Affect' and 'Negative Affect' refer to trait positive and negative affect assessed via the PANAS; Expressivity metrics were assessed by the BEQ. All scores were standardized within sample; identical plots broken down by study can be accessed in the supplement. 'Hi' refers to the cluster of participants who tended to frequently use all five emotion regulation strategies; 'Lo' refers to the cluster of participants who tended to infrequently use all five strategies; 'Mix' refers to the cluster of participants who selectively moderated strategy usage. Order of cluster label pairings in the second row of plots indicate sign of comparison. Cohen's $d$ values reflect an average of estimates across all appropriate samples. Error bars on Cohen's $d$ estimates reflect aggregated analytic 95\% confidence intervals. LAB2 data are not represented here as these metrics were not assessed in that sample. 
Figure 3. Aggregate group differences between clusters on mental health symptoms (clinical symptoms)
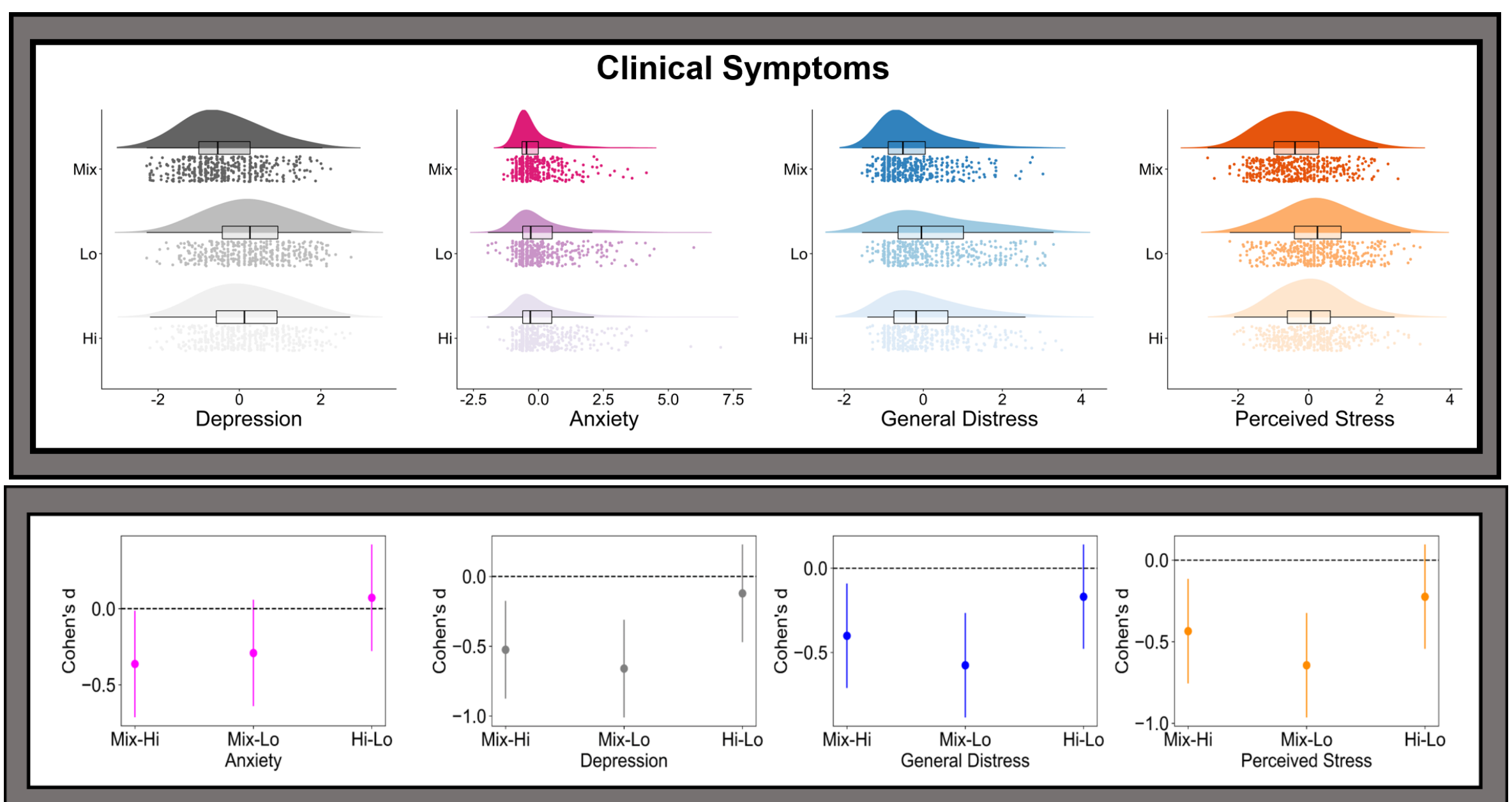

Note. 'Anxiety' refers to scores from the anxious arousal subscale of the Mini-MASQ for ONLINE1-3 and LAB1, and scores from the generalized anxiety subscale of the SCAARED for LAB2; 'Depression' refers to scores from the anhedonic depression subscale of the Mini-MASQ for ONELINE1-3. 'General Distress' refers to scores from the generalized internalizing symptomatology subscale of the MINI-MASQ for ONLINE13 and LAB1. 'Perceived Stress' refers to scores from the PSS for ONLINE1-3 and LAB1. All scores were standardized within sample; identical plots broken down by study can be accessed in the supplement. 'Hi' refers to the cluster of participants who tended to frequently use all five emotion regulation strategies; 'Lo' refers to the cluster of participants who tended to infrequently use all five strategies; 'Mix' refers to the cluster 
of participants who selectively moderated strategy usage. Order of cluster label pairings in the second row of plots indicate sign of comparison. Cohen's $d$ values reflect an average of estimates across all appropriate samples. Error bars on Cohen's $d$ estimates reflect aggregated analytic 95\% confidence intervals. 
Table 1. Characteristics of the six samples of participants.

\begin{tabular}{|c|c|c|c|c|c|c|}
\hline Characteristic & ONLINE1 & ONLINE2 & ONLINE3 & LAB1 & LAB2 & TEST-RETEST \\
\hline Sample Size & $N=401$ & $N=406$ & $N=300$ & $N=127$ & $N=101$ & $N=157$ \\
\hline Sex (Female) & $53.1 \%$ & $50.5 \%$ & $79.7 \%$ & $100 \%$ & $79.2 \%$ & $43.3 \%$ \\
\hline Average Age, SD & $41.16,11.03$ & $41.56,11.61$ & $20.24,2.57$ & $19.59,1.51$ & $18.36,0.48$ & $40.89,11.98$ \\
\hline Race & $\begin{array}{c}\text { Af Am - 7.7\% } \\
\text { Asn - } 7.7 \% \\
\text { NH/PI - } 0.0 \% \\
\text { W - 79.8\% } \\
\text { NA/AN - } 0.2 \% \\
\text { O - } 0.5 \% \\
\text { M - 3.7\% }\end{array}$ & $\begin{array}{c}\text { Af Am - 8.6\% } \\
\text { Asn - 5.2\% } \\
\text { NH/PI - } 0.0 \% \\
\text { W - 81.8\% } \\
\text { NA/AN - } 0.0 \% \\
\text { O - } 1.0 \% \\
\text { M - 3.4\% }\end{array}$ & $\begin{array}{c}\text { Af Am - } 2.3 \% \\
\text { Asn - } 49.3 \% \\
\text { NH/PI - } 0.3 \% \\
\text { W - } 28.3 \% \\
\text { NA/AN - } 1.0 \% \\
\text { O - } 9.0 \% \\
\text { M - 8.0\% }\end{array}$ & $\begin{array}{c}\text { Af Am - } 6.3 \% \\
\text { Asn - } 44.1 \% \\
\text { NH/PI - } 0.8 \% \\
\text { W - 30.0\% } \\
\text { NA/AN - } 0.0 \% \\
\text { O - } 9.4 \% \\
\text { M - } 0.0 \%\end{array}$ & $\begin{array}{c}\text { Af Am - } 10.0 \% \\
\text { Asn - 37.0\% } \\
\text { NH/PI - } 0.0 \% \\
\text { W - } 27.0 \% \\
\text { NA/AN - } 2.0 \% \\
\text { O - } 8.0 \% \\
\text { M - } 12.0 \%\end{array}$ & $\begin{array}{c}\text { Af Am - 8.3\% } \\
\text { Asn - 8.3\% } \\
\text { NH/PI - } 0.0 \% \\
\text { W - } 77.0 \% \\
\text { NA/AN - } 1.9 \% \\
\text { O - } 0.6 \% \\
\text { M - 3.8\% }\end{array}$ \\
\hline $\begin{array}{c}\text { Ethnicity } \\
\text { (Hispanic/Latinx) }\end{array}$ & $9.0 \%$ & $7.1 \%$ & $21.0 \%$ & $18.1 \%$ & $24.0 \%$ & $4.5 \%$ \\
\hline $\begin{array}{l}\text { Mental Health } \\
\text { Assessments }\end{array}$ & All & All & All & All & $\begin{array}{l}\text { Partial Clinical } \\
\text { Symptoms }\end{array}$ & None \\
\hline Longitudinal Data & No & No & No & No & Yes & No \\
\hline
\end{tabular}

Note. 'Sex (Female)' refers to the percentage of participants in each sample which reported being biologically female. 'Average Age, SD' refers to the mean and standard deviation of age in each sample (in years). Race abbreviations are as follows: 'Af Am' refers to African American, 'Asn' refers to Asian/Asian American, 'NH/PI' refers to Native Hawaiian or Other Pacific Islander, 'W' refers to White, 'NA/AN' refers to Native American or Alaskan Native, 'O' refers to a separate, unlisted category, 'M' refers to any mixed race category. Any percentage of racial categories missing from a given study reflects the percentage of participants who gave no answer for race. 'Ethnicity (Hispanic/Latinx)' refers to the percentage of participants in each sample identifying as Hispanic or Latinx. ONLINE1 and ONLINE2 were collected via MTurk, ONLINE 3 was collected via an undergraduate student subject pool for online studies, LAB1 and LAB2 were laboratory studies whose participants were recruited from the UCLA undergraduate student community; TEST-RETEST was collected via MTurk, comprised of a pool of subjects who completed the measure on two separate occasions, approximately two-weeks apart. Demographics for TEST-RETEST are only provided for subjects who 
participated in both timepoints (202 subjects participated at time 1, 158 participated at time 2, 157 participated in both). LAB2 did not have data on affective symptoms, and only partial data on clinical symptoms (depression, anxiety); TEST-RETEST only had E-ERQ data available. 


\section{Supplement For \\ A Data-Driven Typology of Emotion Regulation Profiles}

\section{Guassi Moreira et al.}

\section{Additional Details}

\section{Participants and Sampling Strategy}

We collected six independent samples for this study (ONLINE1, ONLINE2, ONLINE3, LAB1, LAB2, TEST-RETEST). In collecting these six samples, our goals were to ensure generalizability across populations and to obtain multiple independent datasets for analyses (e.g., scale item validation, clustering, out-of-sample predictive validity, etc.). Characteristics of each sample (size, demographics) are listed in Table 1. In the aggregate, we report data from $N=1492$ individuals $(62.5 \%$ Female, mean age $=33.65$ years, age range $=18-82$ years). Note that these samples are comprised of individuals from various ethnic backgrounds and ages. Sample sizes ranged from 101 (LAB2) to 406 (ONLINE2), and were sufficiently powered to uncover clustering solutions on the E-ERQ (Dalmaijer et al., 2020).

Participants were recruited from two populations: Workers from Amazon's Mechanical Turk (MTurk) and undergraduate students at the University of California, Los Angeles (UCLA). Three samples were collected online (three recruited from MTurk, one via UCLA's online undergraduate psychology subject pool), and two were collected via traditional laboratory participation (recruited from community advertisements and the subject pool). Sample sizes were determined a priori and in accordance with available funding for paid participants, available credits for subject pool participants (determined by the university), and logistics idiosyncratic to each sample. LAB1 and LAB2 were part of broader data collection efforts (see refs 78,79 for details), and thus some aspects of their design were not optimized for the current inquiry (e.g., choice of ancillary measure to collect, sample demographics, etc.). Data collection procedures for online and laboratory studies follow below. Across all studies, consent was obtained for all participants in accordance with the policies and approval of the UCLA Institutional Review Board.

Online data collection procedures. For samples collected on MTurk, workers (prospective participants) were presented with a study advertisement asking them to 'answer questions about emotions.' Participants signed up for the study by clicking on an anonymized link that redirected them to the survey (hosted on Qualtrics, an online survey platform). For the sample collected via online subject pool (ONLINE3), prospective participants were presented with a virtually identical study description on UCLA's online experiment management system (SONA) and could enroll by selecting a study slot on the system and clicking on an anonymized link. Regardless of recruitment method, the anonymized link directed participants to read a detailed consent form and affirmatively indicate they agreed to participate in the study. Afterwards, participants completed various self-report instruments (see Materials) and reported demographic information. The order of self-report measurements varied randomly between subjects, and demographic items were always presented last. Inclusion criteria for all online studies were that (i) individuals must be currently residing in the United States (ii) and be at least 18 years of age. An additional criterion - that individuals may not have participated in any other prior study from our laboratory — was imposed for ONLINE2 and TEST-RETEST.

We took several steps to ensure the collection of valid data. First, we included both explicit and implicit attention checks. Explicit attention checks consisted of a multiple choice question (e.g., "what is $13+5$ ?") that occupied an entire survey page. If participants answered incorrectly, they were immediately disqualified from the study. Participants from each sample were presented with one explicit attention check. Implicit attention checks consisted of directions to participants that were embedded amongst the actual items of a given self-report instrument (e.g., 'Rate this item as 'Strongly Agree' would be printed as a survey item). Implicit attention checks were reviewed for accuracy after a respondent completed their 


\section{EMOTION REGULATION TYPES}

survey. Each survey contained eight implicit attention checks; respondent data was rejected and not included in analysis if more than two implicit attention checks were incorrect (the threshold for rejection with implicit attention checks was comparatively lenient since the nature of the check meant a participant could inadvertently select the wrong response option). Responses were also rejected if participants completed the survey in an implausibly fast amount of time (e.g., 90 seconds) or if there was strong evidence that they did not complete the measures seriously (e.g., selected the same response option for all items in a long scale containing reverse worded items).

A random number was generated and displayed on the final page of the online survey, and participants were instructed to submit that number to an experimenter for validation. This step also helped ensure data integrity by limiting the likelihood of artificial (bot) responses. Once validated, participants were compensated ( $\$ 1.75$ for MTurk participants and 1 credit hour for subject pool participants). The surveys typically took participants $15-25$ minutes to complete.

Laboratory data collection procedures. Full details of data collection procedures from in-lab studies are described in ref 79 (LAB1) and ref 78 (LAB2) but are briefly summarized here. Participants for both studies were recruited from the UCLA undergraduate student population (LAB1 via undergraduate psychology subject pool and LAB2 via community advertisements). The primary focus of both studies was to answer orthogonal respective research questions (social influences on emotion regulation via reappraisal in $\mathrm{LAB} 1$, tracking individual differences in various facets of well-being across the college transition in LAB2), and the measures in this report were added as a secondary focus. Given the nature of their respective primary foci, LAB1 only recruited female undergraduate students, and LAB2 only recruited freshman students transitioning to university from high school. In both studies, participants met a trained experimenter in our laboratory, completed the consent process, and completed the survey measures described below ('Materials'). The experimenter unobtrusively monitored each participant to ensure proper compliance. Participants were either compensated with credit hours (LAB1) or money (LAB2). Additionally, LAB2 contained a longitudinal component wherein participants completed brief follow-up assessments of anxiety 30 and 60 days after baseline. These data formed the basis for the longitudinal analyses reported in this report.

\section{Materials}

Emotion Regulation Strategy Usage. Trait emotion regulation usage (sometimes referred to as emotion regulation tendency; McRae, 2013; Silvers \& Guassi Moreira, 2019) was assessed using a novel 22-item self-report measure, the Extended Emotion Regulation Questionnaire (E-ERQ). The E-ERQ is based on the Process Model of Emotion (Gross, 2015), which proposes that different regulatory strategies can be deployed at different points in the unfolding of emotional experience. The traditional Emotion Regulation Questionnaire (ERQ) is a widely-used self-report measure (it has been cited over 10,000 times according to a April 2021 Google Scholar search), which assesses use of two regulatory strategies: cognitive reappraisal and expressive suppression (John \& Gross, 2003). In creating the E-ERQ, we sought to extend the original ERQ to assess the use of five strategies within the Process Model: cognitive reappraisal (6 items, part of original ERQ), expressive suppression (4 items, part of original ERQ), distraction (5 items, novel addition), selective attention (4 items, novel addition), and situation selection (3 items, novel addition). Consistent with the original ERQ, participants were asked to indicate their level of agreement with each item using a 7-point Likert scale ( $1=$ "Strongly disagree", $4=$ "Neutral", $7=$ "Strongly agree"). Items for each of the five strategies were separately averaged to compute trait strategy usage scores, such that higher scores indicate greater trait usage. The measure was extensively validated and found to have desirable psychometric properties (e.g., high reliability, construct validity, discriminant validity, samples wide range of latent trait values, items have high latent trait discrimination). More information about measure creation, validation, as well as the measure itself is included here in the Supplementary Information (Section 3). 


\section{Mental Health Symptoms - Affective Symptoms}

Trait Affect. The PANAS is comprised of a pair of scales designed to measure affect along two major valence dimensions, positive and negative. We instructed participants to indicate their trait affect, meaning they were explicitly told to answer according to how they 'generally' felt or how they felt 'on average'. Participants used a 5-point Likert scale to complete the survey ( $1=$ "Very slightly or not at all", 2 = "A little", 3 = "Moderately", 4 = "Quite a bit", 5 = "Extremely"). The instrument is comprised of 20 items (10 positive, sample items: "Strong", "Excited", "Inspired"; 10 negative, sample items: "Afraid", "Irritable", "Guilty"). Responses for each scale were averaged separately, yielding a trait positive affect score and a trait negative affect score for each participant (greater values indicated greater trait affect). The instrument was administered in all three online samples and one lab sample (4 total), and was found to have excellent multidimensional reliability across all administrations (ONLINE1: $\omega=0.947$; ONLINE2: $\omega=0.950$; ONLINE3: $\omega=0.888$; LAB1: $\omega=0.846$ ).

Emotional Expressivity. The BEQ taps the three facets of emotional expressivity (positive expressivity, negative expressivity, and expressivity strength (i.e., impulse strength)). Results involving the emotional expressivity facet are not reported in the current manuscript for the sake of brevityinterested readers can download our code and data for themselves to examine such effects. Participants use a 7-point Likert scale ( $1=$ "Strongly disagree", $4=$ "Neutral", $7=$ "Strongly agree") to indicate their agreement with 16 statements encompassing the aforementioned three facets (positive, sample item: "I laugh out loud when someone tells me a joke that I think is funny"; negative, sample item: "It is difficult for me to hide my fear"; sample strength item: "I experience my emotions very strongly"). Relevant items were reverse scored and then items corresponding to each facet were averaged into a single score (higher scores indicated greater expressivity). The instrument was administered in all three online samples and one lab sample (4 total), and was found to have excellent multidimensional reliability across all administrations (ONLINE1: $\omega=0.936$; ONLINE2: $\omega=0.930$; ONLINE3: $\omega=0.917$; LAB1: $\omega=0.899$ ).

Sensation Seeking. Participants used a 5-point Likert scale ( 1 = "Strongly disagree", 2 = "Disagree", 3 = "Neither disagree nor agree", 4 = "Agree", 5 = "Strongly agree") to indicate their dispositional levels of sensation seeking (sample items: "I prefer friends who are excitingly unpredictable" and "I like to do frightening things"). Responses were averaged into a single score (higher values indicated greater sensation seeking). This instrument was administered in all five samples, and was found to have adequate reliability across all administrations (ONLINE1: $\omega=0.859$; ONLINE2: $\omega=$ 0.858; ONLINE3: $\omega=0.764$; LAB1: $\omega=0.795$; LAB2: $\omega=.787$ ). Results involving sensation seeking are not reported here for the sake of brevity__interested readers can download our code and data for themselves to examine such effects.

\section{Mental Health Symptoms - Clinical Symptoms}

Internalizing Symptoms. The Mini-MASQ was used to assess internalizing symptoms in most samples. The instrument is an abbreviated version of the MASQ, which assess depressive and anxious symptoms according the tripartite theory of anxiety and depression (Clark \& Watson, 1991). The MiniMASQ taps 'pure' depression (anhedonic depression), 'pure' anxiety (anxious arousal), and general distress (unspecified internalizing symptoms) across a 26 item set. Participants used a 5-point Likert scale ( 1 = "Not at all", 2 = "A little bit", 3 = "Moderately", 4 = "Quite a bit", $5=$ "Extremely") to indicate how often they had been experiencing internalizing symptoms in the past week (anxiety, sample item: "Was trembling or shaking"; depression, sample item: "Felt like nothing was very enjoyable"; general distress, sample item: "Felt discouraged"). Responses were reverse scored where appropriate and items corresponding to each facet were averaged into a single score (higher scores indicated greater internalizing symptoms). The instrument was administered in all three online samples and one lab sample (4 total), and was found to have excellent multidimensional reliability across all administrations (ONLINE1: $\omega=0.963$; ONLINE2: $\omega=0.964$; ONLINE3: $\omega=0.945$; LAB1: $\omega=0.930$ ). 


\section{EMOTION REGULATION TYPES}

As a reminder, LAB2 employed different measures because the initial goal of that study was geared towards answering orthogonal research questions. LAB2, which measures depressive symptoms across 20 items (sample item: "I felt like I could not shake off the blues even with help from my family or friends") with a 4-point Likert scale ( $0=$ "Rarely or none of the time", $1=$ "Some or a little of the time", 2 = "Occasionally or a moderate amount of time", 3 = "Most or all of the time"). Anxiety in LAB2 was assessed using SCAARED. Participants rated the frequency of anxious symptoms over the prior three months (sample item: "It is hard for me to stop worrying") using a 3-point Likert scale ( $0=$ "Not true or hardly every true, 1 = "Somewhat true or sometimes true", 2 = "Very true or often true"). Responses for both scales were reverse scored where appropriate and averaged into a single score for each construct. Both instruments evinced excellent reliability (CESD: $\omega=0.912$; SCAARED: $\omega=0.956$ )

Perceived Stress. The PSS quantifies the extent to which individuals appraise general life events as being subjectively stressful. Participants used a 5-point Likert scale ( 1 = "Never", 2 = "Almost Never", 3 = "Sometimes", 4 = "Fairly Often", 5 = "Very Often") to indicate how often in the past month they had experienced thoughts and feelings individuals commonly have when stressed (sample items: "In the past month, how often have you felt difficulties were piling up so high that you could not overcome them?" and "In the past month, how often have you been able to control irritations in your life?"). Responses were reverse scored where appropriate and averaged to yield a single score (higher values indicated greater stress). This instrument was administered in all three online samples and one lab sample (4 total), and was shown to have excellent reliability across all four administrations (ONLINE1: $\omega=0.963$; ONLINE2: $\omega=0.964$; ONLINE3: $\omega=0.945$; LAB1: $\omega=0.930$ ).

\section{Psychometric Validation of the E-ERQ}

Summary. Validation of the novel E-ERQ required four steps: creating an initial measure (approximately 15 items for each of the three novel strategies), removing items that exhibited weak associations with the intended latent trait (i.e., a given regulation strategy; the final measure contained 5 distraction, 4 selective attention, and 3 situation selection items), characterizing the factor structure of the five strategies (as indicated by observed responses to items), and determining whether the measure possessed sufficient reliability. Overall, the measure was shown to have adequate to excellent psychometric properties. Across three validation samples (ONLINE1, ONLINE2, ONLINE3), E-ERQ items showed strong associations with their respective latent traits, and provided information (i.e., precision) over a wide range of their respective latent traits. Each strategy assessed by the E-ERQ displayed unidimensionality, indicating that subscales could be used independently and discretely tapped a unique strategy.

Factor analyses using all strategies in a single model revealed that a bifactor structure best fit the data across the three validation samples. However, bifactor structures can yield higher fit statistics even when said structures do not accurately model the data (Bornovalova et al., 2020). Our models showed some evidence of a bifactor structure, but this was not absolute and we note competing factor structures (correlated factors model, higher-order factor model) were also insightful insofar that they revealed complimentary factor structure information. Our general interpretation of these results is that the strategies tapped by the E-ERQ are not 'effectively unidimensional', and that there likely exists some kind of hierarchical structure amongst the strategies. Whether said structure is strongly bifactor or some alternate hierarchical structure is subject to future work and theoretical scrutiny. This is noteworthy because (i) the factor structure of these five emotion regulation strategies has not previously been examined and this knowledge is intrinsically helpful for future work (e.g., further encourages researchers to assess multiple strategies) and (ii) it signifies the items map onto individual strategies in addition to general tendencies.

Finally, model-based reliability analyses showed the novel E-ERQ components evinced suitable to excellent reliability, both cross-sectionally (in terms of MacDonald's $\omega$ ), but also longitudinally, in the 


\section{EMOTION REGULATION TYPES}

form of test-retest reliability. Full details and statistical output of this process can be accessed in the Supplementary Information (Section 3). Correlations among strategies across all samples are included here in the Supplementary Information (Section 4).

Item Creation and Feedback. We initially generated approximately 20 items for each of the three understudied emotion regulation strategies (distraction, selective attention, situation selection), with the goal of retaining approximately 4-6 items per strategy. The language and content of the items was modeled after the existing ERQ, containing references to general emotional experiences (e.g., "When I want to control my emotions..."), as well as both positive and negative scenarios ("When I want to feel more positive emotion..." and "When I want to feel less negative emotion"). In order to maintain consistency with the ERQ, in addition to practical reasons, we did not consider items where individuals were aiming to upregulate negative emotion or downregulate positive emotion. All items directly or indirectly tapped trait-level tendency to engage in a different strategy, and explicitly did not ask about whether individuals felt they were effective at using a given strategy (capacity). A majority of items were phrased in the affirmative direction, and a subset of items were worded negatively for reverse scoring. This latter subset initially was included to reduce the confounding influence of positive response (acquiescence) bias, although no reverse scored items proved to be psychometrically sound and were dropped from the final version of the measure (none of the original ERQ items are negatively worded, so we do not feel this represents a major problem).

Prior to collecting the first validation sample, we solicited feedback from members of our research group that were not involved in the project. We asked our colleagues within the research group to evaluate whether each item appropriately tapped its intended emotion regulation strategy, adequately conveyed capture information about tendency (and not capacity), was easy to understand, was consistent with the style of the original ERQ, and captured frequent/likely real world scenarios (if applicable). In addition to asking researchers within our laboratory, we also approached other colleagues in the department and asked them for feedback on clarity. Approximately five items from each strategy were cut after this stage, having been deemed to be unclear, confusing, or irrelevant. This left approximately 15 items per strategy to be validated.

Validation Step 1 - Item-Level Evaluation via IRT. Following item creation, we proceeded to validate each individual item via IRT. This occurred in an iterative fashion by collecting a dataset, performing IRT on the items of each strategy, and dropping the worst items until we approximated our target of 4-6 items per strategy ${ }^{4}$.

Items were evaluated using IRT. IRT is an item-level analysis that estimates the association between a latent trait and the likelihood of endorsing a given item using a generalized linear model (Reise et al., 2005; Reise \& Waller, 2009). Put differently, IRT assumes that individual differences in an underlying latent trait cause variance in item responses. Such associations in IRT are characterized by two important concepts - location (sometimes also known as difficulty) and discrimination. Location describes the level of latent trait at which the probability of endorsing an item is .5. A greater location parameter means an item is 'more difficult' as only individuals with greater scores on the latent trait are expected to endorse the item. Because the items of the E-ERQ are polytomous, we used a graded response model (GRM). The GRM allows one to model the likelihood of endorsing a particular scale option or higher, yielding $m-1$ location parameters (where $m$ is equal to the number of response categories). Because E-ERQ items use a 7-point scale, our models contained six location parameters $\left(b_{1-6}\right)$. Discrimination $(a)$ refers to the strength of association between the latent trait and responses on a given item; in other words, it is a slope parameter.

\footnotetext{
${ }^{4}$ In the event this process yielded a strategy with a number of items far below this threshold (e.g., 1 or 2), we planned to create and validate additional items. This was ultimately rendered unnecessary as we were able to retain a reasonable number of items per scale.
} 
Item locations and discriminations form the bedrock for two important units of IRT analysis - the item response curve and item information curve (Reise et al., 2005; Reise \& Waller, 2009). The item response curve summarizes a mathematic function between the latent trait and observed responses to a given scale. Item locations and discriminations comprise the parameters that give each item their own unique individual item response curve. Item response curves resemble a sigmoid that tracks the likelihood of endorsing a particular item category (y-axis) across the range of the latent trait (x axis). In turn, the item response curve can be converted to an item information curve ${ }^{5}$. In IRT, the concept of 'information' is related to precision. Items that are more precise in their measurements are thought to convey more information because they are better able to differentiate among individuals across the range of the latent trait. Item information curves thus track the precision of a given item (y-axis) across the range of the (xaxis). It is customary for items in the same instrument to differ in their item response and information curves. This can be advantageous because the aforementioned IRT item properties are additive - using items to calculate a composite (sum, average) means that item-level properties are combined and reflected in said composite, and therefore items with different location parameters and information functions can contribute information when used as part of a composite. For instance, a certain item may discriminate between individuals who are very high on the latent trait and those who are not. Simply using this item would not be useful in discriminating between individuals who are very low on the latent trait and those who are not. Similarly, a certain item may convey much information (i.e., be very precise) about individuals who score below average on the latent trait, but convey little to no information about individuals who score above average. Additivity allows researchers to pool the psychometric properties of different items by combining them into a composite score.

We used item level response and information curves, in addition to their location and discrimination parameters, to decide which items to keep or drop from the measure during validation. We also considered fit statistics ( $\chi^{2}$ and RMSEA) when deciding, but did not use them slavishly as they can misrepresent important graphical trends in the data - for the purpose of brevity, we omit these statistics until the final validation. We also display scale-level response and information curves to highlight the overall psychometric properties of each strategy's scale at each stage of validation. All IRT models were fit using the mirt()function from R's 'mirt' package (Chalmers, 2012). Maximum likelihood estimation was conducted using the BFGS optimizer. Latent trait values were assumed to follow a standard normal distribution $(\theta \sim N(0,1))$; models were estimated along the range $[-6,6]$ for latent traits. We fit exploratory unidimensional GRMs, one for each scale of the E-ERQ, on the first validation sample (ONLINE1). Our rationale for fitting models for reappraisal and suppression was that they could (i) serve as benchmarks and (ii) it would be beneficial to the field to obtain additional psychometric assessments on the traditional ERQ items since they have not been validated widely with IRT (Brandao et al., 2017; Ireland et al., 2018; Preece et al., 2021). Statistical output is listed in Supplementary Table 2. Items that were dropped at this phase of analysis were those with very weak discrimination parameters (usually less than 1.5), poor breadth in their location parameters (i.e., covered less than $\sim 3$ units on the latent trait), or had response/information curves that showed patently poor psychometric attributes (e.g., flat or negative response curve, completely flat information curve). In all, we dropped six distraction items, eight selective attention items, and six situation selection items. We kept items that did not have abjectly poor psychometric properties in order to avoid being too conservative in the first iteration of item validation, knowing that we were likely to make cuts in future iterations.

After this, we collected another independent validation sample (ONLINE2) to further validate the measure, this time imposing stricter criteria for keeping items (i.e., stronger discriminations, wider locations, 'sharper' curves, were needed this time). This time we fit confirmatory, unidimensional GRM models to the items belonging to each strategy (same estimation details as above). Statistical output for

\footnotetext{
${ }^{5}$ Item response and item information curves are technically referred to as category response and category information curves when dealing with polytomous data. However, here we use 'item' instead of 'category' for the sake of simplicity.
} 


\section{EMOTION REGULATION TYPES}

these models is listed in Supplementary Table 3. For this round, we kept items that generally had discrimination values greater than 2, location parameters that spanned approximately 3.5/4.0 units of the latent trait, and had response/information curves that were 'sharper' than before. This resulted in cutting three items from the distraction scale, two items from the selective attention scale, and three items from the situation selection scale. This left us with a number of items in each scale consistent with our target (4-6 items).

Our final iteration of item validation was to confirm the psychometric properties observed in ONLINE2 in a third, independent sample from a novel population. To that end, we recruited a third such sample from UCLA's undergraduate subject pool (ONLINE3). We again ran the same confirmatory IRT models for the six strategies. Across the items, discrimination parameter estimates generally ranged from 2 to 3 , and location parameters generally spanned approximately 4 units of the latent trait. Visual inspection of item information and response curves confirmed that none of the items were unacceptable and confirmed that at all were at least adequate. Importantly, the novel items were comparable, or in some cases better, than the benchmark original ERQ items. Item-level fit statistics were also favorable. RMSEA values respectively ranged between .000-.034, .000-.022, and .017-.043 for the distraction, selective attention, and situation selection items; none of the item-level chi-square statistics were significant for any items across the three strategies. Overall, these analyses show that our novel E-ERQ items are psychometrically comparable to the original ERQ items and are appear to be apt at measuring their intended constructs with reasonable precision for approximately $95 \%$ of individuals (corresponding to a span of $\sim 4$ units on the latent trait). Location and discrimination parameters for all three rounds of this validation step are tabulated in Supplementary Tables 2-4.

Validation Step 2 - Uncovering Factor Structure. After validating E-ERQ items using IRT, we used the final items to validate the factor structure of the five regulation strategies captured by the EERQ. This validation step was comprised of two components. We first tested whether the items indicating each strategy's latent factor were indeed unidimensional, and then we sought to analyze the factor structure among the strategies. We note that much of the statistical output in this section has been condensed or unreported in the interest of brevity. However, all analysis code and data are publicly available for readers interested in replicating and viewing the full statistical output.

To verify undimensionality among each strategy, we ran confirmatory factor analysis (CFA) using the $\mathrm{cfa}($ ) function from R's 'lavaan' package (cite). Maximum likelihood estimation was used, and all observed and latent variances were standardized. We thus ran fifteen unidimensional CFA models (one for each strategy, one set of models for each validation sample). Across all three samples, factor loadings for reappraisal ranged between $.507-.891$, RMSEA values ranged between $.087-.163$, TLI values ranged between $.862-.970$, and CFI values ranged between $.917-.982$. Factor loadings for suppression ranged between $.447-.896$, RMSEA values ranged between $.072-.129$, TLI values ranged between .923 - .976, and CFI values ranged between .974 - .992. Factor loadings for distraction ranged between .648 .785 , RMSEA values ranged between $.107-.165$, TLI values ranged between $.890-.945$, and CFI values ranged between $.919-.972$. Factor loadings for selective attention ranged between $.626-.830$, RMSEA values ranged between $.000-.143$, TLI values ranged between $.935-1.003$, and CFI values ranged between .935 - 1.00. Factor loadings for suppression ranged between $.665-.891$, fit statistics could not be computed since there were only three situation selection items. Overall, these models provide moderate evidence that each strategy's scale is unidimensional in isolation. We also note that the lower range of the fit statistics, which sometimes dipped below acceptable thresholds, were likely influenced by relatively small number of items for each strategy (especially RMSEA), and thus we do not place too much stock in conventional guidelines for evaluating fit indices.

After this step, we turned our attention towards evaluating the factor structure of all five strategies tapped by the E-ERQ. We enumerated three theoretically plausible models - A correlated factors model wherein each item loads onto a single latent factor corresponding to its emotion regulation strategy and 


\section{EMOTION REGULATION TYPES}

said latent factors are allowed to correlate with one another; a hierarchical model wherein the aforementioned correlations between said latent factors are further modeled as loading onto a single second order (hierarchical) latent factor (e.g., a general 'emotion regulation tendency' factor); a bifactor model where each item loads onto a general emotion regulation tendency factor as well as a groupspecific factor (a factor indicating its intended emotion regulation strategy) reflecting common variance among items of a strategy after accounting for the general factor. Notably, the bifactor and hierarchical factor models are mathematical equivalents - they simply offer different interpretations of correlations among latent factors (Bornovalova et al., 2020). Therefore, both models can offer unique insights into a factor structure of interest such as variance accounted for by general vs group sources, and how well group factors contribute to a supraordinate factor.

Across all three samples, the bifactor model consistently evinced the best fit statistics, better than the correlated factors model (CFI range: .899-.933; TLI range: .882-.922; RMSEA range: .069-.078) and the hierarchical model (CFI range: .878-.924; TLI range: .862-.914; RMSEA range: .073-.080) (Supplementary Table 5). Importantly, the factor loadings for the general and group factors were sufficiently high to reasonably justify a bifactor structure (Supplementary Table 5). However, this did not mean the bifactor structure was without its limitations. The items for suppression did not load highly on the general factor, and the items were selective attention were only slightly better. Moreover, half of the reappraisal items did not load well onto their respective group factor. On the one hand, these results indicate that the five strategies measured by the E-ERQ are undergirded by both a strong general factor corresponding to generic emotion regulation tendency and strategy specific factors (i.e., strategy specific tendency contributes to variance on observed items over and above the general factor). On the other hand, however, our application of the model showed that some strategies were not optimally configured for this framework.

Overall, we are left to conclude that the bifactor model does an adequate job at modeling the factor structure of the five regulatory strategies, as measured by the E-ERQ, while leaving open the possibility for the existence of more complex factor structures. For instance, perhaps the bifactor model best applies to reappraisal, distraction and situation select. Indeed, this is corroborated by factor loadings from the hierarchical factor model, which show said three strategies loaded highly on the second order factor (reappraisal: 1.151-1.877; distraction: 1.596-1.884; situation selection: 0.817-2.100) while suppression and selective attention loaded poorly (suppression: -0.023-0.349; selective attention: 0.7460.897). This could also be caused by a coarse taxonomic distinction between the two sets of strategies, or be an artifact resulting from the omission of regulation strategies that were not measured here. Alternately, it is possible a bifactor model does indeed best describe the data, but more many more items — or items with superior psychometric properties - may be needed to fully sample the latent factor space (9).

Validation Step 3 - Assessing Reliability. Having evaluated both the psychometric properties and factor structure of the E-ERQ items, we moved on to our final validation step by examining the measure's reliability. We relied on four metrics to assess reliability: $\omega, \omega$-h, $\omega$-strategy, and Pearson's $r$ in a testretest sample. Coefficient $\omega$ is a conceptual analog to Cronbach's $\alpha$, with key advantages. While both metrics describe the percentage of variance due to systematic sources (presumably from loadings between observed indicators and a latent factor of interest), $\alpha$ makes a number of assumptions that are often unrealistic (unidimensionality, equivalence among factor loadings, etc.). While we developed the E-ERQ so that researchers may use individual strategies as unidimensional modules, the collective measure itself is not unidimensional and factor loadings are certainly not equal between items. Therefore, $\omega$ is more appropriate. One advantage of $\omega$ is that it is model-based, meaning a statistical model — carefully informed by theory - is used to estimate parameters that will be used to calculate $\omega$. This 'respects the data' by using data-driven information about systematic sources of variance (latent factors), instead of relying on summary statistics and un-tested theoretical assumptions. 


\section{EMOTION REGULATION TYPES}

In addition to $\omega$, we estimated $\omega$-h- - what proportion of variance in E-ERQ scores is accounted by the general factor alone - as well as $\omega$-strategy - what proportion of variance among scores for a specific strategy (e.g., average reappraisal scores) are caused by the general factor and the respective strategy. These metrics were estimated across all samples. Finally, we estimated the test-retest reliability of a measure in an additional online sample (TEST-RETEST). The sample $(N=156)$ was collected via MTurk and administered the E-ERQ approximately two weeks apart. Pearson's correlation coefficient $(r)$.

Model-based reliability statistics ( $\omega$ family coefficients) are listed in Supplementary Table 6. Each time point of the TEST-RETEST sample is given twice since both administrations contained individuals who only participated at one time point. Notably, $\omega$ is excellent for all samples and $\omega$-h is similarly high, suggesting aggregate scores on the E-ERQ are reliable and could serve as appropriate multidimensional composites. $\omega$-strategy scores were also high, further showing that researchers can use the E-ERQ for computing scores on specific strategies of interest (as we do here for clustering purposes). Notably, the LAB2 sample contained some relatively outlying estimates, but we reason this is due to inefficient calculation of model parameters given the relatively low sample size (101 subjects) and we note that the scores are not low enough to be inappropriate for analysis. Finally, test-test reliabilities are depicted in Supplementary Table 7. Values range from acceptable to borderline excellent, providing further evidence that the E-ERQ taps stable, trait-like emotion regulation strategy usage.

\section{Analytic Procedure}

K-Medoids Clustering. K-Medoids clustering was implemented using the Partitioning Around Medoids algorithm (PAM) via the pam()function in R's 'cluster' package. Our first step was to determine the optimal number of clusters. We did this using two metrics: the gap statistic and the within-cluster sum of squares (Tibshirani et al., 2001). The general method for determining the optimal number of clusters is to compute the respective metric over a number of possible clusters $(k)$ and then select the cluster number that optimizes said statistic. Both of these statistics are optimized when adding an additional cluster does little or nothing to change the value of the statistic (e.g., the gap statistic does not appreciably increase, or the within cluster sum of squares does not decrease). In other words, the optimal number of clusters is decided at the 'elbow' of the visualizations of these statistics. Using R's 'clus' and 'facto_extra' packages, we calculated and visualized the gap statistics and total sums of squares across our datasets for $k=2$ to 6 . Our ceiling of $\mathrm{k}=6$ clusters was informed by prior work in personality psychology (Gerlach et al., 2018). We did not evaluate $k=1$ in order to avoid the risk of a false negative (i.e., clusters do not exist; 90). We reasoned that if our clustering procedure resulted in a false positive, this would be evinced by a seemingly random pattern of cluster means among the five strategies and cluster differences in mental health symptoms.

Justification for using K-Medoids, as well as our assumptions about our data, follow. K-Medoids is also relatively parsimonious in that extra variance parameters and cluster weights for each observation are not estimated. We therefore deliberately chose K-Medoid clustering because it is less sensitive to outliers than K-Means and more parsimonious than Gaussian mixture models. Consistent with prior seminal studies on trait-level typologies (Gerlach et al., 2018), this approach assumes that each emotion regulation strategy constitutes a dimension on which individuals can vary, and in turn that clusters of individuals occupy distinct areas in a five-dimensional space. The five dimensions correspond with the five emotion regulation strategies measured by the E-ERQ. Position in the five dimensional space was measured using mean scores from each strategy assessed by the E-ERQ, meaning each subject had a row vector with five entries corresponding to mean scores on trait-level reappraisal, suppression, distraction, selective attention, and situation selection usage. We elected to use observed mean scores instead of extracting factor scores from a factor analysis model because the latter method is frequently biased and inefficient. 
We conducted several robustness checks. First, to ensure the optimal number of clusters in the dataset was not simply due to noise in the data, we re-ran our analyses after reducing the dataset's dimensionality, via t-distributed stochastic neighbor embedding (tSNE) from five to two ( $t$-distributed stochastic neighbor embedding). t-SNE data reduction has been shown to reduce noise and improve detection of clusters. Our results were not appreciably different when using t-SNE. Second, we also ran analyses with the next best value for $k$ to determine whether our subsequent results could not be explained by a more parsimonious solution ${ }^{6}$. As noted in the results section, our findings held when using these robustness checks. Third, we first pooled data from five samples (ONLINE1-3, LAB1-2) together, re-ran the clustering algorithm specifying $k=3$, and computed the proportion of participants whose cluster assignment remained stable. Furth, we ran the clustering algorithm on both timepoints of the TESTRETEST sample and computed the short-term (approximately two weeks), temporal stability of cluster assignment (again as the proportion of participants whose cluster assignment did not change). Fifth, we assessed the stability of our clustering solution via bootstrapping, implemented with the fpc package's clusterboot()function ( $\mathrm{B}=100$ bootstraps, recommended by the package authors). This procedure works by resampling data and then comparing similarity between the original and boostrapped clusters. Jaccard similarity values greater than 0.5 are considered to mean a cluster is stable; values greater than .75 indicate very good stability (see Hennig, 2007). Similarity values for all samples were generally considered acceptable (ONLINE1: 0.756 - 0.830; ONLINE2: 0.736 - 0.761; ONLINE3: 0.551 - 0.610; LAB 1: 0.445 - 0.656; LAB2: 0.663 - 0.869). Notably, samples with lower similarity values also had smaller sample sizes, suggesting their relatively lower stability might be an artifact of not having as many participants. Finally, we used sample statistics to repeatedly simulate E-ERQ data (2500 replications per sample) without imposing any sort of clustering structure to create a null distribution and then examined the probability of observing the cluster means for each strategy reported in the main document under the null. Collectively, these results generally suggest the pattern of cluster means obtained here were unlikely to be due to chance (ONLINE1: RP 0.028, SP 0.024, DS 0.098, SA 0.089, SS 0.016; ONLINE2: RP 0.038, SP 0.020, DS 0.050, SA 0.086, SS 0.035; ONLINE3: RP 0.016, SP 0.005, DS 0.029, SA 0.045, SS 0.020; LAB1: RP 0.012, SP 0.445, DS 0.317, SA 0.548, SS 0.138; LAB2: RP 0.010, SP 0.001, DS 0.019, SA 0.023, SS 0.122).

Searching for Mechanisms Underlying Cluster Differences in Affective Symptoms and Mental Health Symptoms. We provide more details on our ridge regression implementation here. Ridge regression, which is different from traditional least squares methods in that it applies a user-specified penalty $(\lambda)$ to coefficients and systematically biases them towards zero (see McNeish, 2015) ${ }^{7}$, was used for two key reasons. First, use of some emotion regulation strategies were highly correlated (e.g., distraction and reappraisal) and ridge regression yields more efficient, and thus more stable, parameter estimates in the face of high multi-collinearity compared to the least squares method. Second, parameter estimates from ridge regression are more generalizable to unseen data (i.e., there will be less error in predicting new cases based on ridge regression parameters in comparison to those obtained with least squares) and are thus of greater value for future applications (e.g., predicting adjustment from regulation strategies using a pre-trained model in clinical settings). Ridge regression analyses were conducted using R's 'glmnet' package. Using the package's cv.glmnet() function, we performed 10-fold cross-validation

\footnotetext{
${ }^{6}$ Another advantage of doing this would be to determine whether a different, yet nevertheless systematic pattern of findings emerged. If this were indeed the case-i.e., a set of cohesive findings across all datasets for both clustering solutions-it would imply degeneracy among hypothetical dimensionalities used to define the typology and could pose an identifiability problem. Fortunately, this was not the case in the current study.

7 It might be more intuitive or helpful to consider ridge regression from a Bayesian perspective. Instead of conceptualizing coefficient estimation as involving a penalty term, one can think of it as placing a zerocentered prior on the regression coefficients and therefore limiting the magnitude of their extremities.
} 
to identify an optimal value for the penalty term $(\lambda)$. We performed inference on the resulting coefficients by computing $95 \%$ percentile bootstrapped confidence intervals (10,000 iterations).

Growth Curve Modeling Specifications. We conducted linear growth curve modeling analyses in a multilevel modeling framework with R's 'nlme' package to examine whether cluster membership influenced longitudinal mental health outcomes and, similarly, whether individual differences in trait strategy usage among the five strategies predicted longitudinal outcomes. We focused on anxiety in the LAB2 sample. This sample was comprised of $\mathrm{N}=101$ undergraduate students surveyed up to three times during their first year of college (292 measurements total across all individuals). Measurement occasions occurred approximately 30 days between each other.

The following describes our modeling approach for examining longitudinal associations between cluster membership and anxiety. Anxiety was predicted as a function of time (coded as days since subject-specific baseline, baseline = zero) and cluster membership (dummy coded, Lo as the reference group). We also entered biological sex (dummy coded, female $=0$, male $=1$ ) and the academic quarter the participant enrolled in the study (dummy coded, fall $=0$, winter $=1$ ). Finally, because some data collection occurred during the COVID-19 pandemic, we adjusted for whether participants provided any data during the pandemic (dummy coded, all data collected prior to COVID-19 $=0$, at least one datum collected during COVID-19=1). This variable was defined using the date UCLA cancelled in-person instruction (March 13 ${ }^{\text {th }}, 2020$ ).

Multilevel models typically assume uncorrelated level-1 (within-person) residuals, yet this is unrealistic in the context of growth curve modeling where data are presumed to have temporal structure (Singer \& Willett, 2003). To correct for potential autocorrelation among residuals, a first order autoregressive structure was used to help estimate the level-1 error variance/covariance structure. In doing so, we assumed covariances between temporally adjacent level-1 errors were greater than those which were not temporally adjacent (e.g.., covariance between errors at lag $=1>$ covariance between errors at lag $=2$ ). The form of the model is notated below.

Within-Person (Level-1):

$$
\text { Anxiety }_{t i}=\pi_{0 i}+\pi_{l i} \text { Time }_{t i}+e_{t i}
$$

Between-Person (Level-2):

$$
\begin{aligned}
& \pi_{0 i}=\gamma_{00}+\gamma_{10} \mathrm{Mix}_{i}+\gamma_{20} \mathrm{Hi}_{i}+\gamma_{30} \text { Sex }_{i}+\gamma_{40} \text { Quarter-Start }_{i}+\gamma_{50} \text { Any-COVID } i+u_{0 i} \\
& \pi_{1 i}=\gamma_{10}+u_{1 i}
\end{aligned}
$$

Anxiety $_{t i}$ refers to self-reported loneliness or anxiety on the $t$-th day since baseline for the $i$-th individual. The first between-person equation represents main effects of between person predictors (cluster membership, indicated by the set of Mix and Hi dummy codes), as well as fixed effect intercept. The second line represents a fixed effect of linear growth. The $u_{0 \mathrm{i}}$ term represents the stochastic component of random intercepts, conditional upon all between person predictors. The $u_{l i}$ term represents the stochastic component of random growth trajectories. Last, $e_{t i}$ denotes within-subject error when predicting a given outcome as a function of time and between-person predictors. We tested for the possibility of a cross-level interaction between cluster membership and time, such that cluster membership would influence growth trajectories. Such an interaction was not significant and was dropped from the model. Therefore, this model tests the degree to which baseline differences in cluster membership remain constant over time in the presence of similar growth trajectories for all groups.

A second set of models was estimated to quantify the temporal stability of individual strategyoutcome associations. These set of models was similar to the prior set. The only difference is in the equation for random intercepts: 


$$
\pi_{0 i}=\gamma_{00}+\gamma_{10} R P_{i}+\gamma_{20} S P_{i}+\gamma_{30} D S_{i}+\gamma_{40} S A_{i}+\gamma_{50} S S_{i}+\gamma_{60} S_{e x}+\gamma_{70} \text { Quarter-Start }_{i}+\gamma_{80} \text { Any-COVID }{ }_{i}+u_{0 i}
$$

Fixed effects $\gamma_{10}-\gamma_{50}$ represent main effects of the five emotion regulation strategies studied here, indicating the association between a given strategy and a given outcome at any time. We again tested cross-level interactions between time and each emotion regulation strategy. A significant interaction implies individual differences in strategy usage correspond to individual differences in linear growth trajectories. Significant interactions are unpacked in Supplementary Figure 7, where we split the level 2 moderator (strategy) at three levels and examined change over time at each level.

\section{Section 5 - Individual Strategy-Symptom Association Results}

Associations between Individual Emotion Regulation Strategies and Affective Symptoms. To further probe the cluster results above, we evaluated patterns of association between each of the five emotion regulation strategies (while adjusting for use of other strategies) and the four affective symptoms (Figure 4). Consistent with prior work (Brockman et al., 2017; Gross \& John, 2003), reappraisal was broadly associated with adaptive affective symptoms, including higher positive trait affect and positive expressivity, and less trait negative affect, and negative expressivity. Also consistent with prior work (Brockman et al., 2017; Kelley et al., 2019), suppression was broadly associated with maladaptive affective symptoms, including increased trait negative affect, and less trait positive affect, positive expressivity, and negative expressivity. Distraction usage showed partially overlapping and partially distinct results from suppression, such that it was positively associated with trait negative affect and negative expressivity, and inversely associated with trait positive affect. Situation selection usage showed partially overlapping and partially distinct results from reappraisal, such that it was positively associated with trait positive affect, and positive expressivity. Selective attention did not have strong associations with any affective symptoms. All of these associations statistically adjust for all other strategies.

Associations between Individual Emotion Regulation Strategies and Clinical Symptoms. The five emotion regulation strategies also showed unique associations with the four clinical symptoms (Figure 5). Reappraisal showed robust inverse associations with all four indicators of poor mental health - anxiety, depression, general distress, and perceived stress. Suppression was generally associated with poor wellbeing outcomes in all domains (except anxiety), albeit not as strongly. Distraction was also associated with worse well-being, and this relation tended to be slightly more robust than suppression. With the exception of a modest positive association with anxiety, selective attention did not show strong associations with the mental health symptoms, nor did situation selection. 
Supplementary Figure 1. Visualizations of Exploratory IRT results from the first iteration of the E-ERQ (ONLINE1)

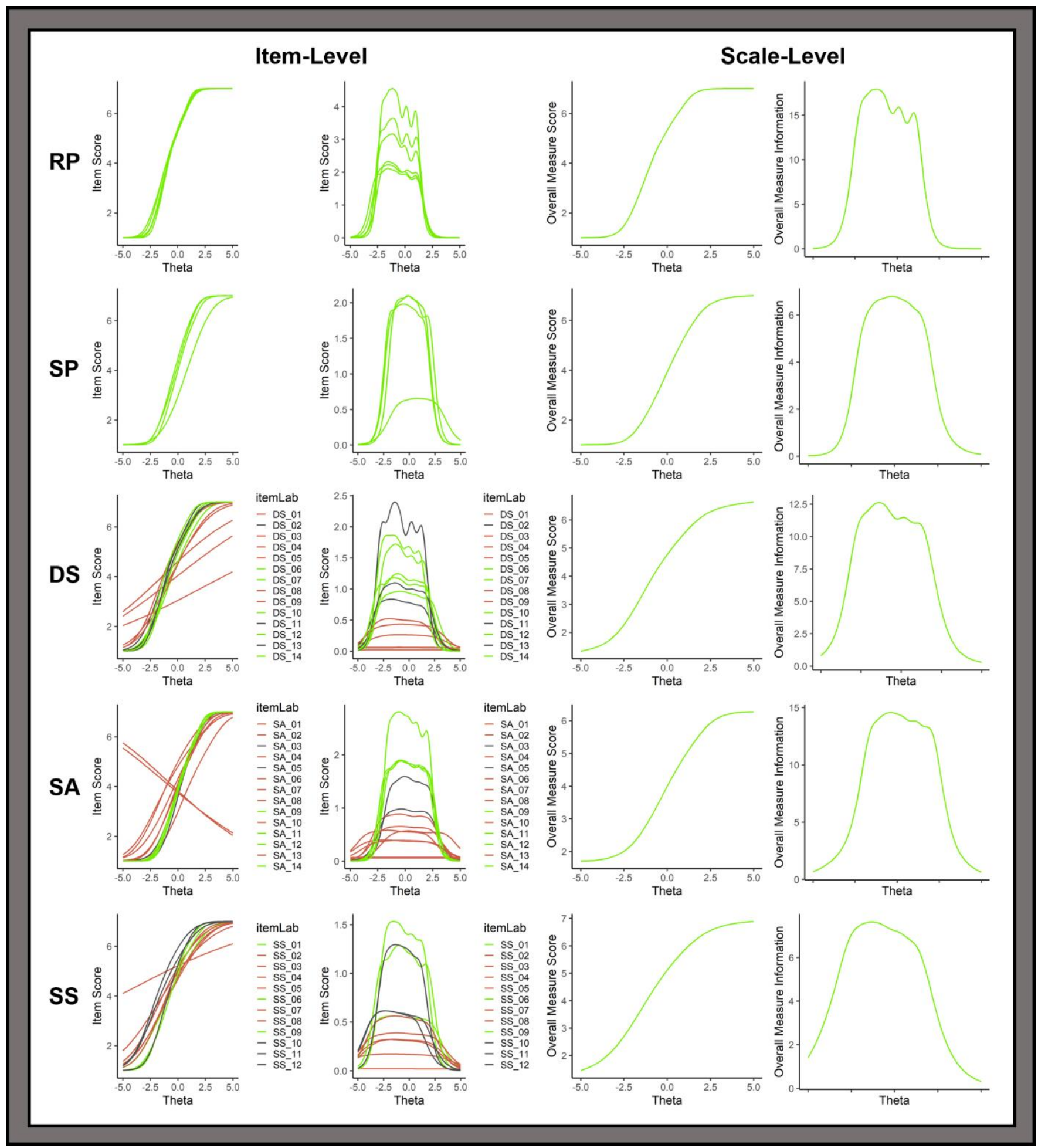

Note. All analyses implemented in R's MIRT package. 'RP' refers to Reappraisal items; 'SP' refers to Suppression items; 'DS' refers to Distraction items; 'SA' refers to Selective Attention items; 'SS' refers to Situation Selection items. Reappraisal and Suppression items were originally developed by Gross \& 
John (2003); IRT models were run on RP and SP items primarily for benchmarking purposes. The following items were discarded after this round of validation: DS - 01, 03, 04, 05, 08, 09; SA - 01, 02, 04, 06, 07, 08, 10, 13; SS - 02, 03, 04, 05, 07, 08. DS, SA, and SS items here are numbered sequentially for convenience; item numbering changes across each of the three validation studies. Exploratory unidimensional IRT models were run for each strategy (i.e., this figure reflects 5 separate IRT models). Plots in the two left-most columns depict item-specific curves; plots in the two right-most columns depict scale-level curves. Response curves are shown in the first and third columns, plotting the strength of relationship between a given item or scale and the latent trait $(\theta)$; information curves are shown in the second and fourth columns, plotting the degree of information (precision) associated with a given item or scale over the range of the latent trait $(\theta)$. 
Supplementary Figure 2. Visualizations of Confirmatory IRT results from the second iteration of the EERQ (ONLINE2)

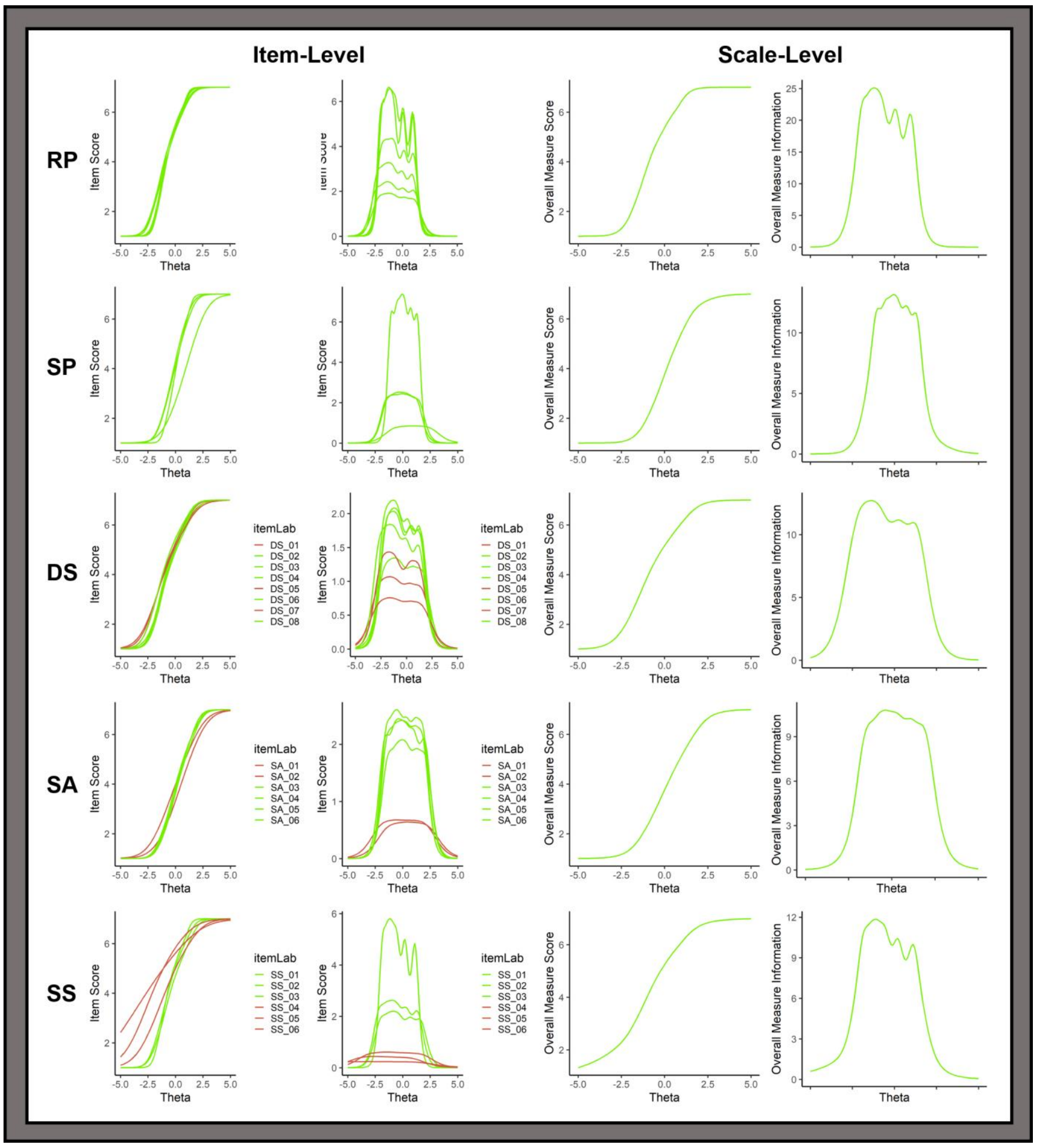

Note. All analyses implemented in R's MIRT package. 'RP' refers to Reappraisal items; 'SP' refers to Suppression items; 'DS' refers to Distraction items; 'SA' refers to Selective Attention items; 'SS' refers 
to Situation Selection items. Reappraisal and Suppression items were originally developed by Gross \& John (2003); IRT models were run on RP and SP items primarily for benchmarking purposes. The following items were discarded after this round of validation: DS - 01, 05, 07; SA - 01, 02; SS -04, 05, 06. DS, SA, and SS items here are numbered sequentially for convenience; item numbering changes across each of the three validation studies. Confirmatory unidimensional IRT models were run for each strategy (i.e., this figure reflects 5 separate IRT models). Plots in the two left-most columns depict itemspecific curves; plots in the two right-most columns depict scale-level curves. Response curves are shown in the first and third columns, plotting the strength of relationship between a given item or scale and the latent trait $(\theta)$; information curves are shown in the second and fourth columns, plotting the degree of information (precision) associated with a given item or scale over the range of the latent trait $(\theta)$. 
Supplementary Figure 3. Visualizations of Confirmatory IRT results from the final iteration of the E-ERQ (ONLINE3)

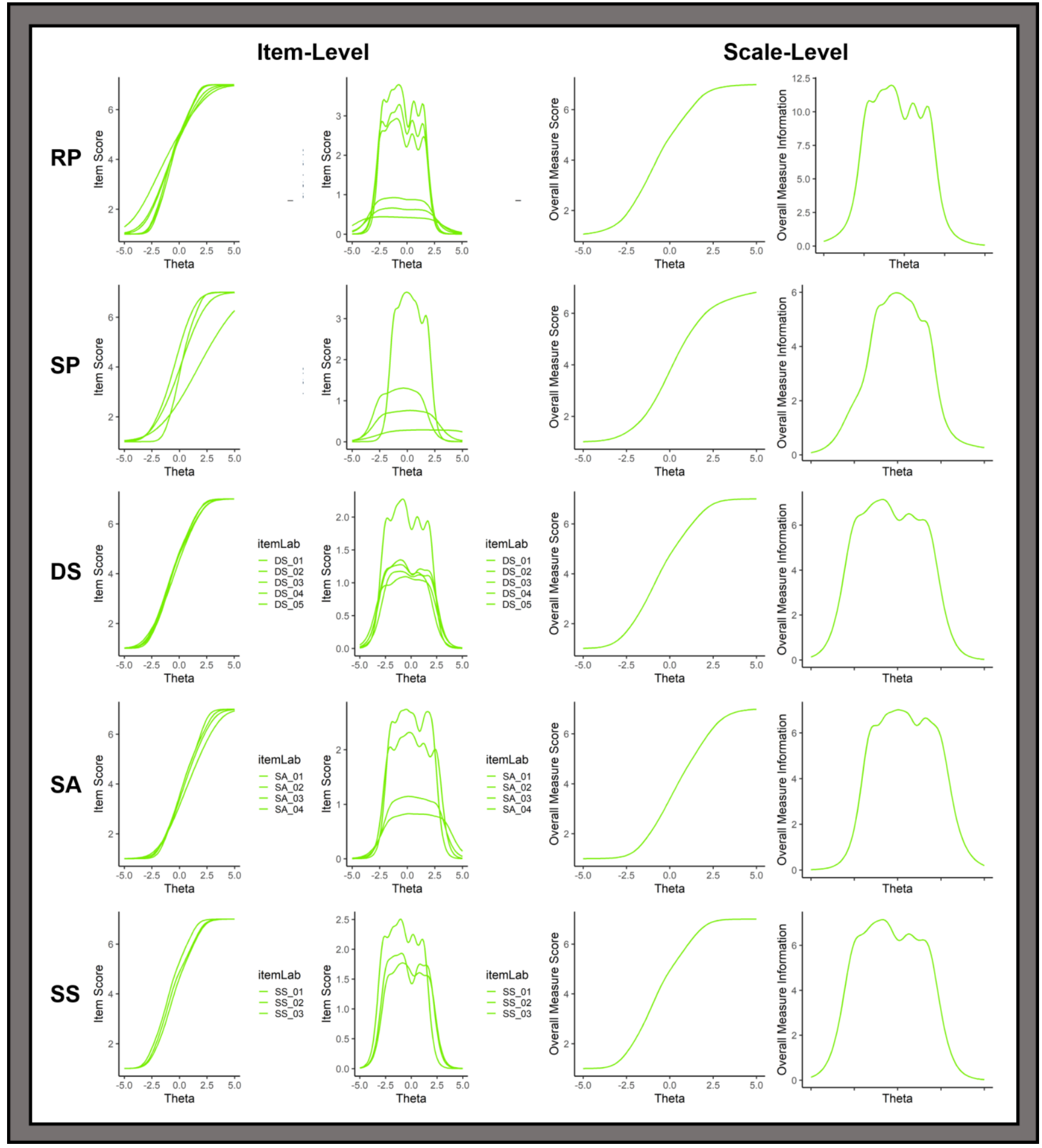

Note. All analyses implemented in R's MIRT package. 'RP' refers to Reappraisal items; 'SP' refers to Suppression items; 'DS' refers to Distraction items; 'SA' refers to Selective Attention items; 'SS' refers to Situation Selection items. Reappraisal and Suppression items were originally developed by Gross \& 
John (2003); IRT models were run on RP and SP items primarily for benchmarking purposes. The following items were discarded after this round of validation: DS - 01, 03, 04, 05, 08, 09; SA - 01, 02, 04, 06, 07, 08, 10, 13; SS - 02, 03, 04, 05, 07, 08. These are the final items for DS, SA, and SS, and their numbering matches that in Supplementary Table 1. Confirmatory unidimensional IRT models were run for each strategy (i.e., this figure reflects 5 separate IRT models). Plots in the two left-most columns depict item-specific curves; plots in the two right-most columns depict scale-level curves. Response curves are shown in the first and third columns, plotting the strength of relationship between a given item or scale and the latent trait $(\theta)$; information curves are shown in the second and fourth columns, plotting the degree of information (precision) associated with a given item or scale over the range of the latent trait $(\theta)$. 
Supplementary Figure 4. Strategy-level associations between five emotion regulation strategies and mental health symptoms (based on affective symptoms)

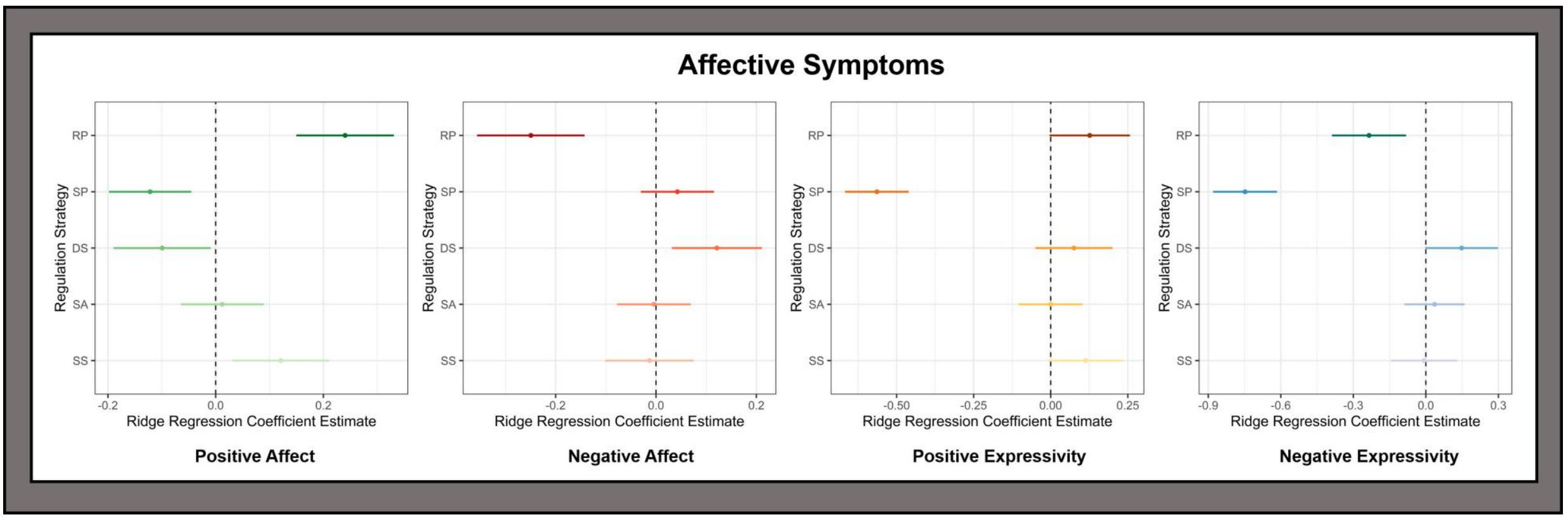

Note. 'RP' refers to Reappraisal; 'SP' refers to Suppression; 'DS' refers to Distraction; 'SA' refers to Selective Attention; 'SS' refers to Situation Selection. Strategy usage assessments reflect mean scores from the novel E-ERQ. 'Positive Affect' and 'Negative Affect' refer to trait positive and negative affect assessed via the PANAS; Expressivity metrics were assessed by the BEQ. Ridge regression coefficients depicted here were obtained by averaging coefficients from all the relevant samples; identical plots broken down by study can be accessed in the supplement. LAB2 data are not represented here as these metrics were not assessed in that sample. Error bars reflect aggregated percentile bootstrapped 95\% confidence intervals. 


\section{EMOTION REGULATION TYPES}

Supplementary Figure 5. Strategy-level associations between five emotion regulation strategies and mental health symptoms (clinical symptoms)

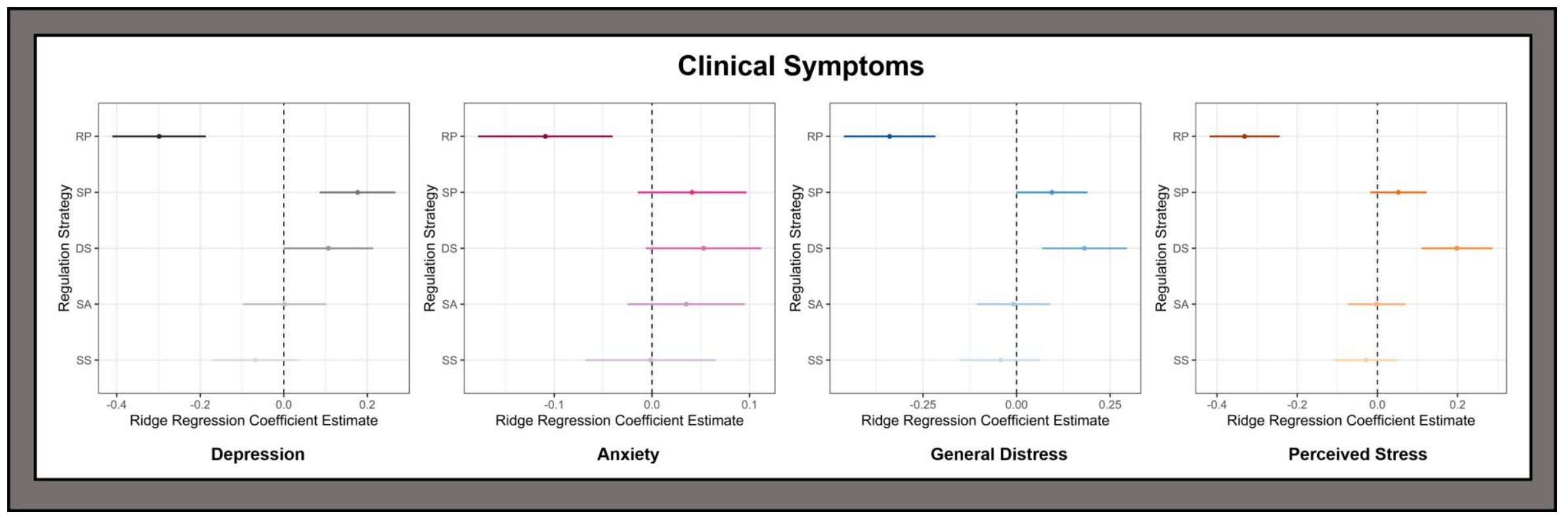

Note. 'RP' refers to Reappraisal; 'SP' refers to Suppression; 'DS' refers to Distraction; 'SA' refers to Selective Attention; 'SS' refers to Situation Selection. Strategy usage assessments reflect mean scores from the novel E-ERQ. 'Anxiety' refers to scores from the anxious arousal subscale of the Mini-MASQ for ONLINE1-3 and LAB1, and scores from the generalized anxiety subscale of the SCAARED for LAB2; 'Depression' refers to scores from the anhedonic depression subscale of the Mini-MASQ for ONELINE1-3. 'General Distress' refers to scores from the generalized internalizing symptomatology subscale of the MINI-MASQ for ONLINE1-3 and LAB1. 'Perceived Stress' refers to scores from the PSS for ONLINE1-3 and LAB1. Ridge regression coefficients depicted here were obtained by averaging coefficients from all the relevant samples; identical plots broken down by study can be accessed in the supplement. Error bars reflect aggregated percentile bootstrapped $95 \%$ confidence intervals. 
51 EMOTION REGULATION TYPES

Supplementary Figure 6. Out-of-sample predictive accuracy predicting mental health symptoms from the set of emotion regulation strategies
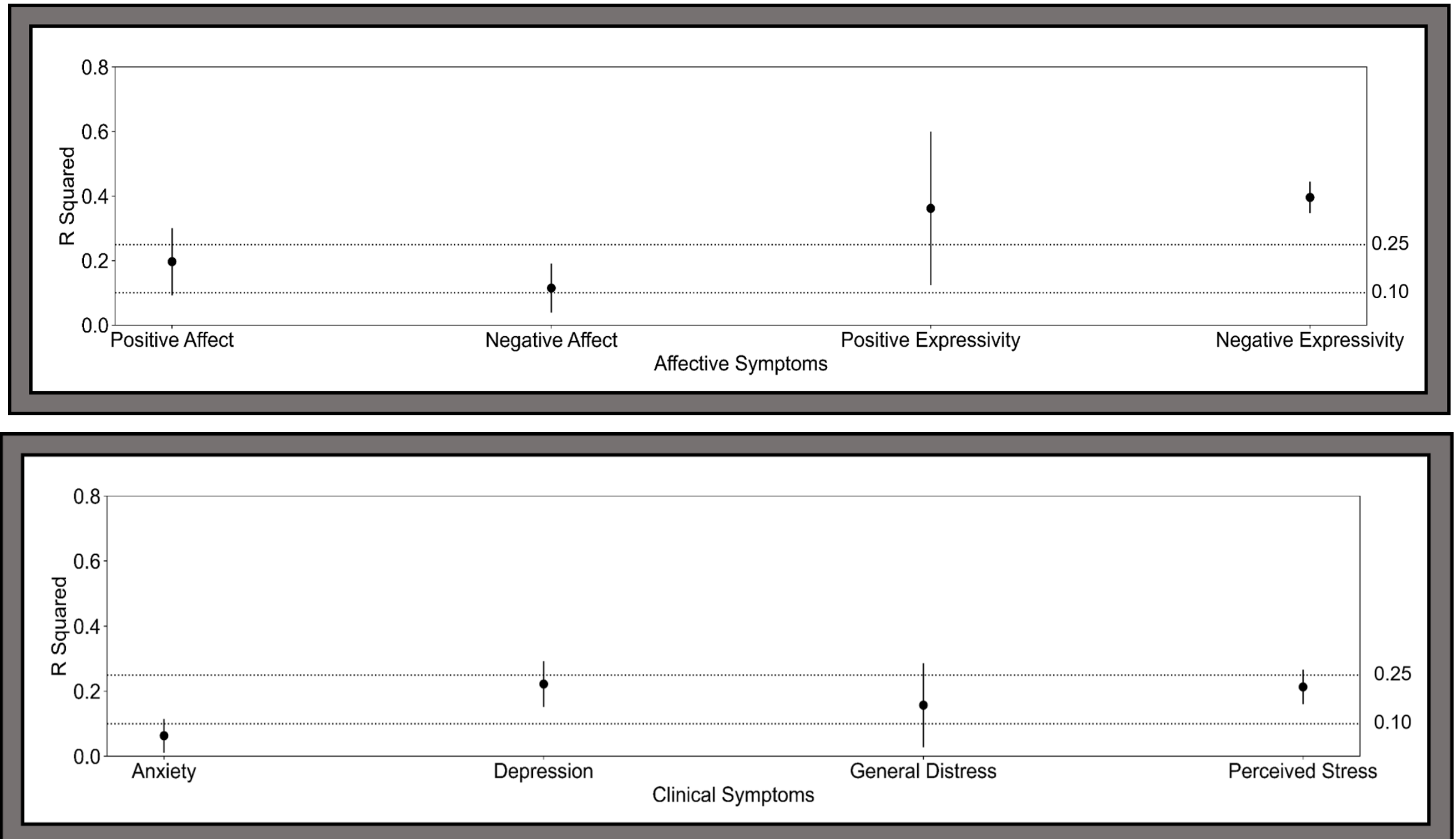

Note. Scores obtained using leave-one-sample-out cross-validation. Point estimates were obtained by averaging over $R^{2}$ on when predicting each 
held-out sample (testing data) from parameters estimated all other samples (training data). Error bars reflect $2 * \mathrm{SD}$ of each variable's set of $R^{2}$ scores. Dotted lines correspond to .10 and .25 , conventional guidelines for medium and large effect sizes. 
Supplementary Figure 7. Raincloud plots of mental health symptoms (affective symptoms) by cluster and sample

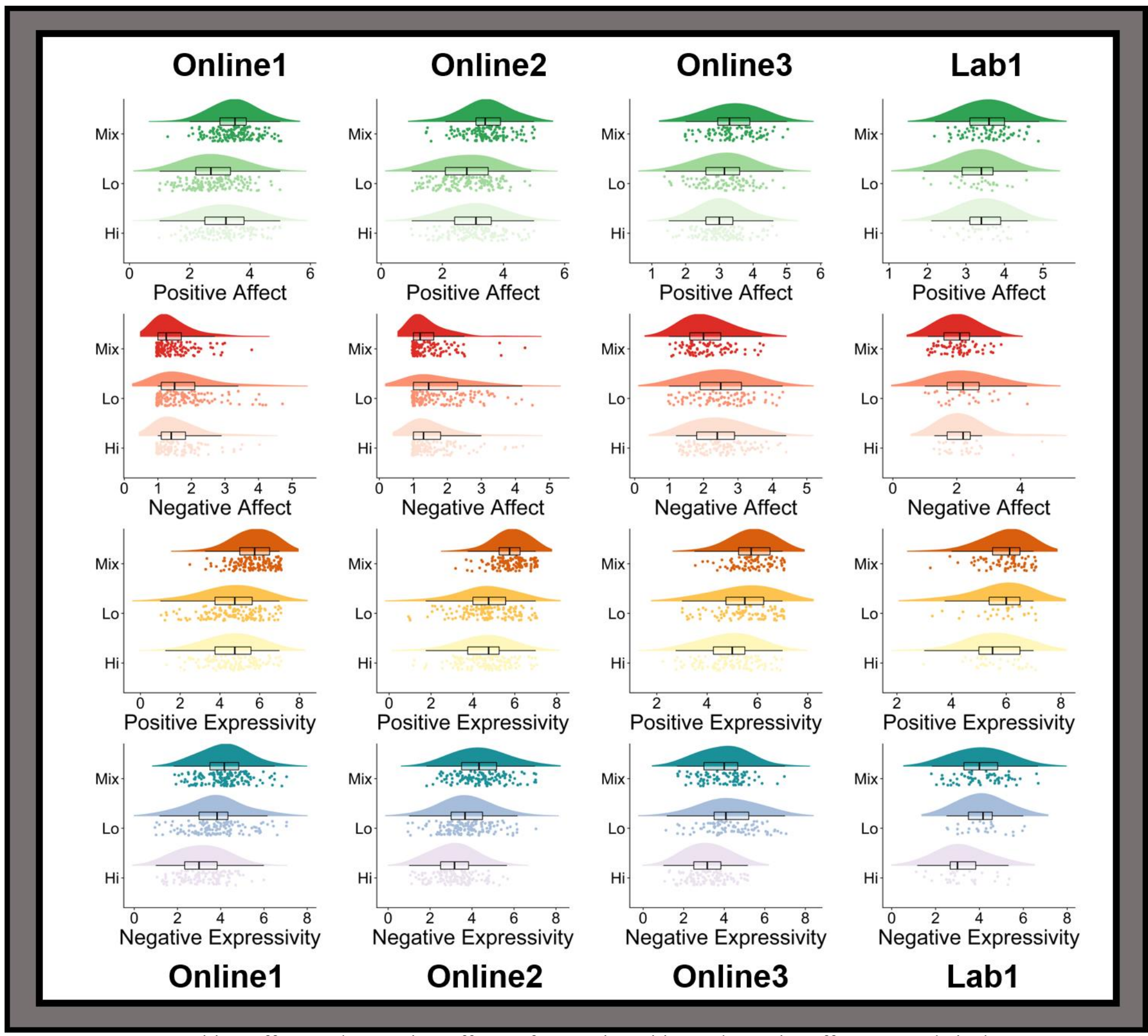

Note. 'Positive Affect' and 'Negative Affect' refer to trait positive and negative affect assessed via the PANAS; Expressivity metrics were assessed by the BEQ. All scores were standardized within sample. 'Hi' refers to the cluster of participants who tended to frequently use all five emotion regulation strategies; 'Lo' refers to the cluster of participants who tended to infrequently use all five strategies; 'Mix' refers to the cluster of participants who selectively moderated strategy usage. LAB2 data are not represented here as these metrics were not assessed in that sample. 
Supplementary Figure 8. Raincloud plots of mental health symptoms (clinical symptoms) by cluster and sample

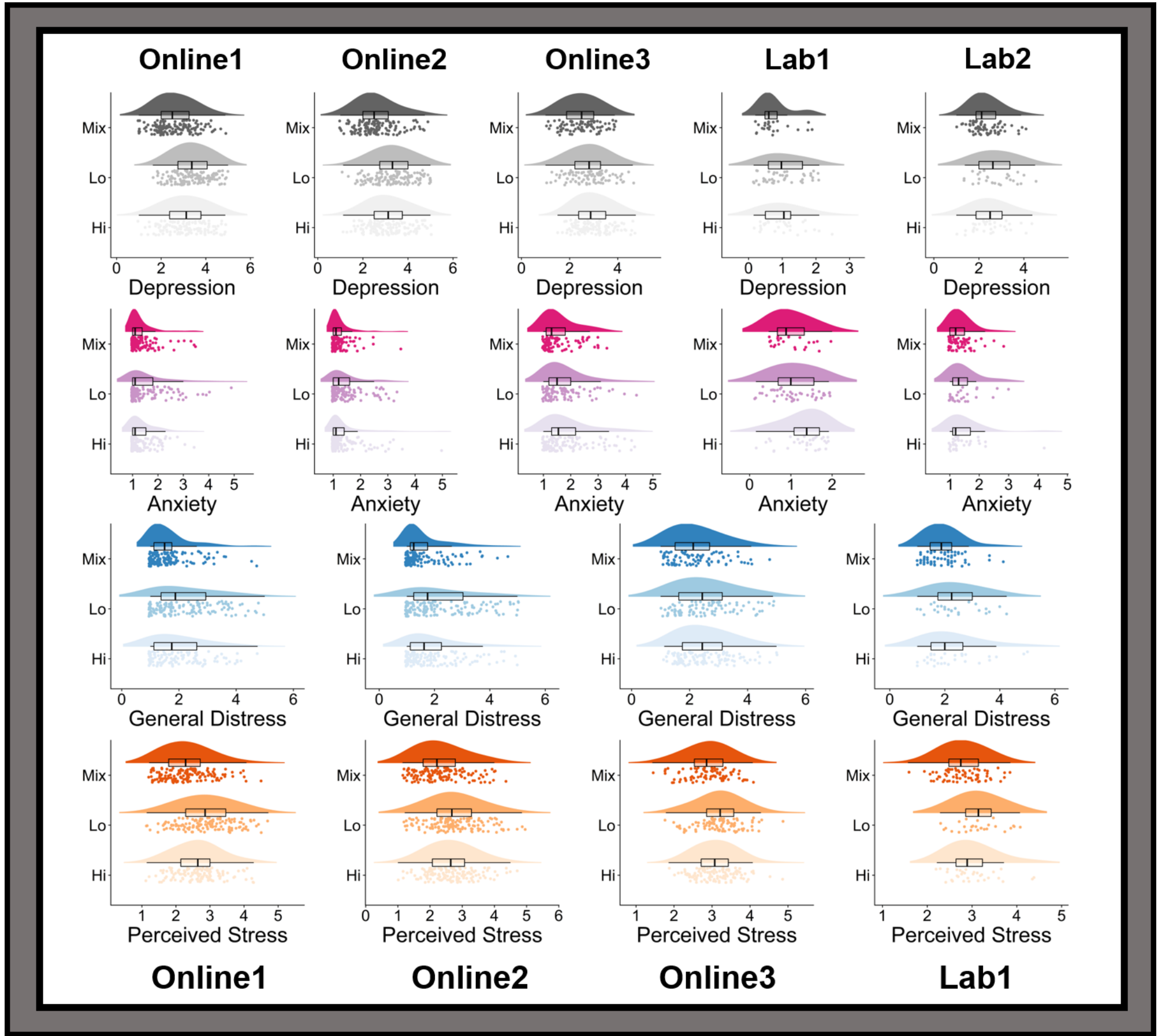

Note. 'Anxiety' refers to scores from the anxious arousal subscale of the Mini-MASQ for ONLINE1-3 and LAB1, and scores from the generalized anxiety subscale of the SCAARED for LAB2; 'Depression' refers to scores from the anhedonic depression subscale of the Mini-MASQ for ONELINE1-3. 'General Distress' refers to scores from the generalized internalizing symptomatology subscale of the MINIMASQ for ONLINE1-3 and LAB1. 'Perceived Stress' refers to scores from the PSS for ONLINE1-3 and LAB1. 'Hi' refers to the cluster of participants who tended to frequently use all five emotion regulation 


\section{EMOTION REGULATION TYPES}

strategies; 'Lo' refers to the cluster of participants who tended to infrequently use all five strategies; 'Mix' refers to the cluster of participants who selectively moderated strategy usage. 


\section{EMOTION REGULATION TYPES}

Supplementary Figure 9. Associations between strategies and mental health symptoms (affective symptoms) by sample

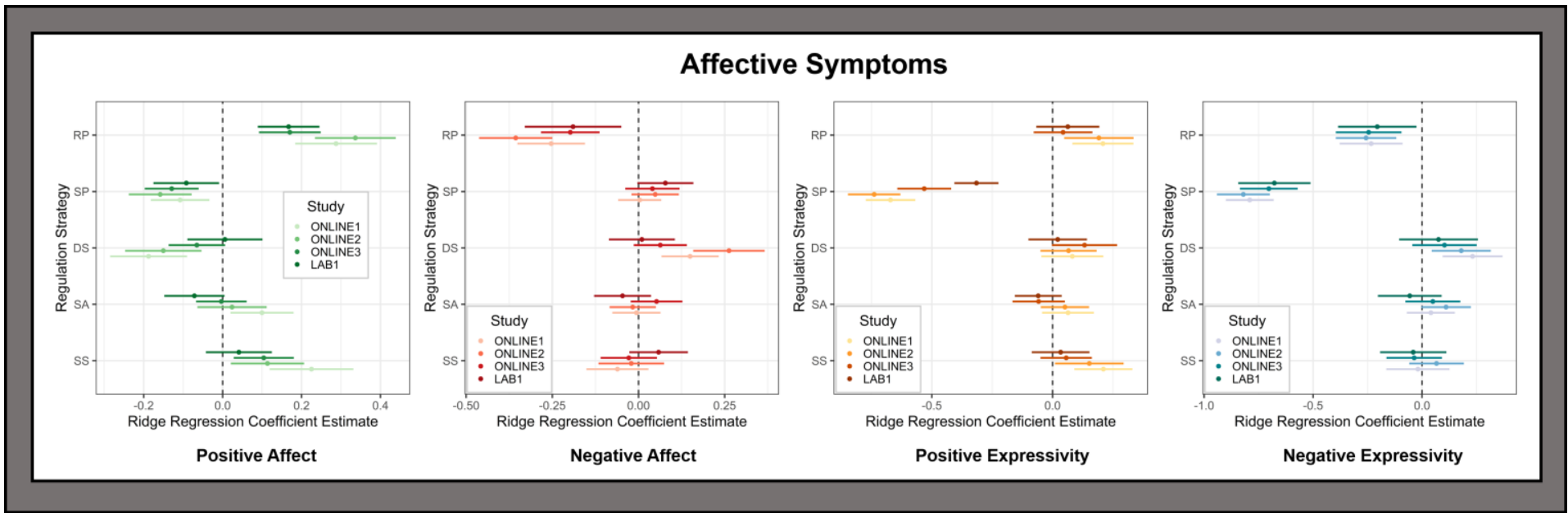

Note. 'RP' refers to Reappraisal; 'SP' refers to Suppression; 'DS' refers to Distraction; 'SA' refers to Selective Attention; 'SS' refers to Situation Selection. Strategy usage assessments reflect mean scores from the novel E-ERQ. 'Positive Affect' and 'Negative Affect' refer to trait positive and negative affect assessed via the PANAS; Expressivity metrics were assessed by the BEQ. LAB2 are not reflected in these analyses. Error bars reflect percentile bootstrapped $95 \%$ confidence intervals. 


\section{EMOTION REGULATION TYPES}

Supplementary Figure 10. Associations between strategies and mental health symptoms (clinical symptoms) by sample

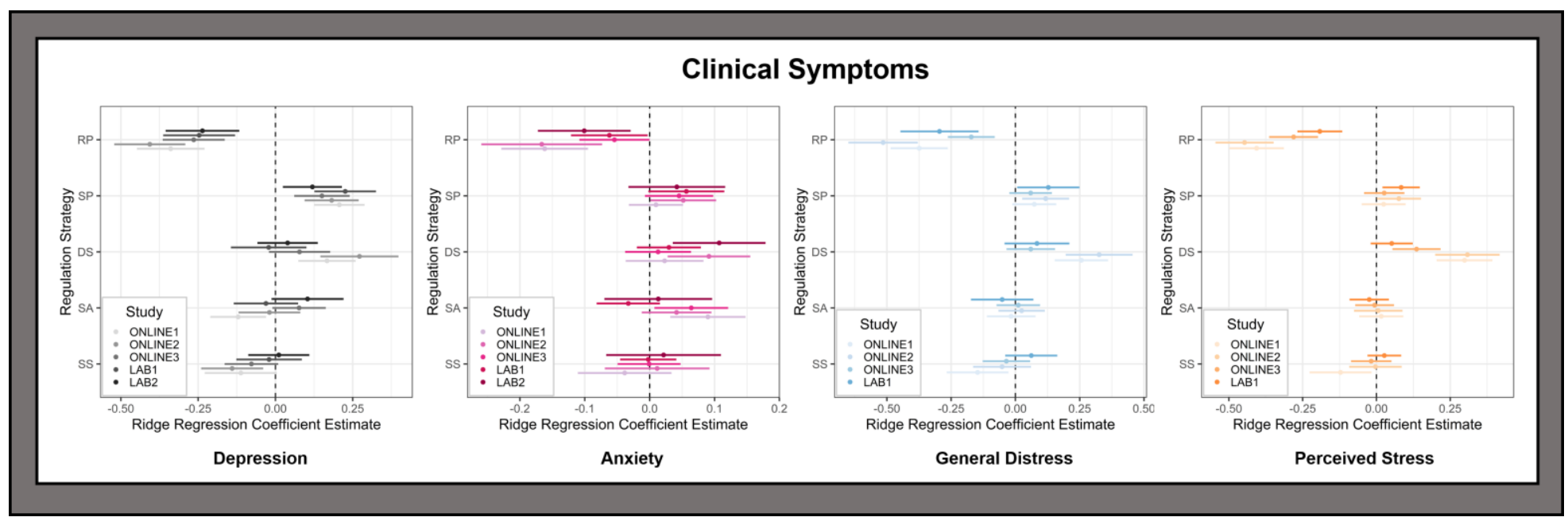

Note. 'RP' refers to Reappraisal; 'SP' refers to Suppression; 'DS' refers to Distraction; 'SA' refers to Selective Attention; 'SS' refers to Situation Selection. Strategy usage assessments reflect mean scores from the novel E-ERQ. 'Anxiety' refers to scores from the anxious arousal subscale of the Mini-MASQ for ONLINE1-3 and LAB1, and scores from the generalized anxiety subscale of the SCAARED for LAB2; 'Depression' refers to scores from the anhedonic depression subscale of the Mini-MASQ for ONELINE1-3. 'General Distress' refers to scores from the generalized internalizing symptomatology subscale of the MINI-MASQ for ONLINE1-3 and LAB1. 'Perceived Stress' refers to scores from the PSS for ONLINE1-3 and LAB1. Error bars reflect percentile bootstrapped 95\% confidence intervals.

Supplementary Figure 11. Trajectories of anxiety for each cluster. 


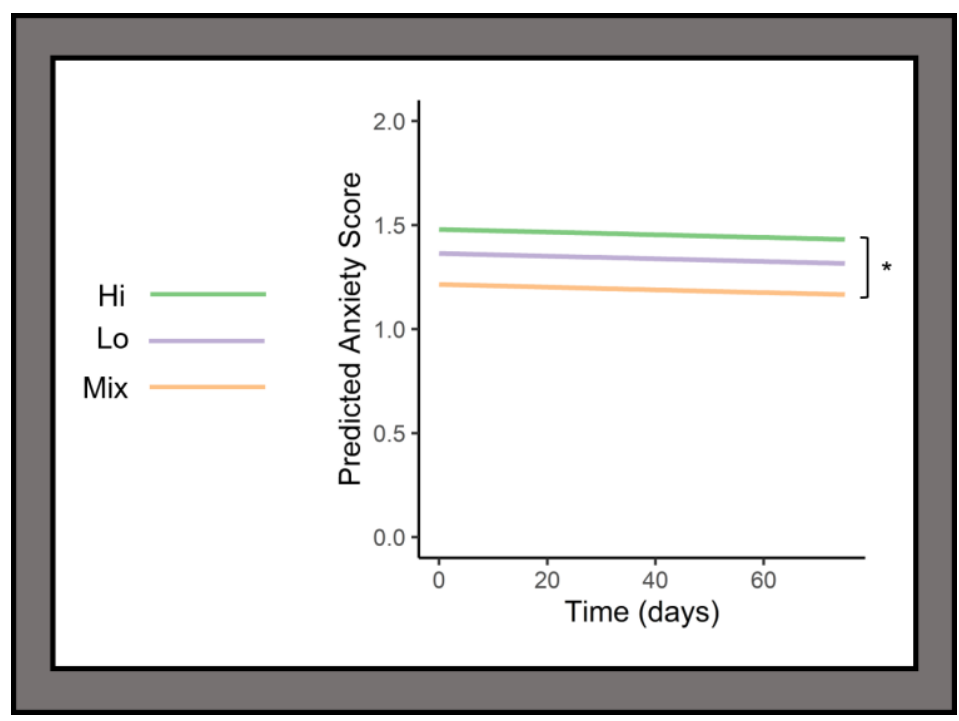

Note. ${ }^{*} p<.05, * * p<.01, * * * p<.001$. 'Hi' refers to the cluster of participants who tended to frequently use all five emotion regulation strategies; 'Lo' refers to the cluster of participants who tended to infrequently use all five strategies; 'Mix' refers to the cluster of participants who selectively moderated strategy usage. 


\section{EMOTION REGULATION TYPES}

Supplementary Figure 12. Trajectories of anxiety across levels of different regulatory strategies

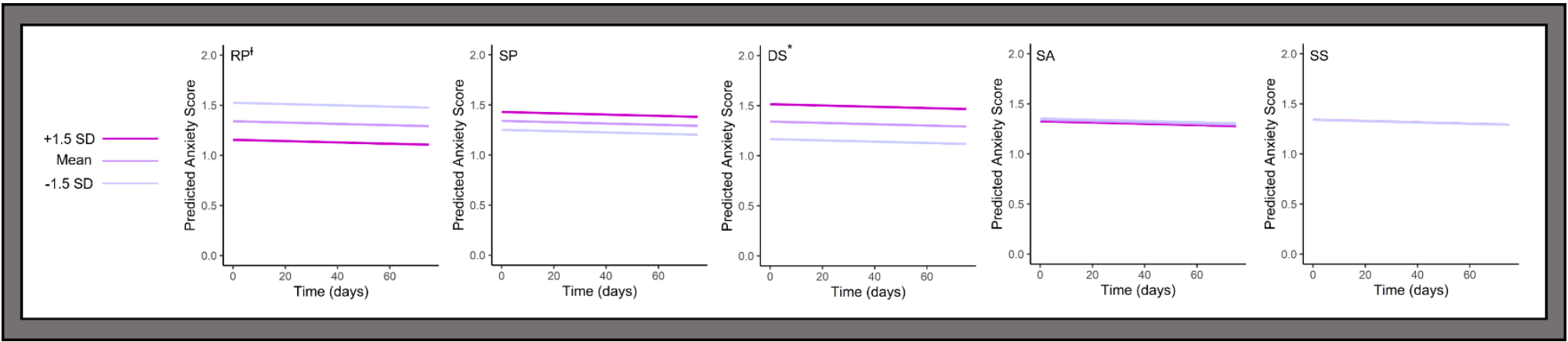

Note. $\mathrm{I} p<.10, * p<.05, * * p<.01, * * * p<.001$.'RP' refers to Reappraisal; 'SP' refers to Suppression; 'DS' refers to Distraction; 'SA' refers to Selective Attention; 'SS' refers to Situation Selection. Strategy usage assessments reflect mean scores from the novel E-ERQ. 
Supplementary Table 1.The novel Extended Emotion Regulation Questionnaire (E-ERQ)

\begin{tabular}{l|l}
\hline Strategy - Item Number & \multicolumn{1}{c}{ Item Text } \\
\hline RP -01 & When I want to feel a more positive emotion (such as joy), I change what I'm thinking about. \\
RP -02 & When I want to feel less negative emotion (such as sadness or anger), I change what I'm thinking about. \\
RP -03 & When I'm faced with a stressful situation, I make myself think about it in a way that helps me stay calm. \\
RP -04 & When I want to feel more positive emotion, I change the way I'm thinking about the situation. \\
RP -05 & I control my emotions by changing the way I think about the situation I'm in. \\
RP -06 & When I want to feel less negative emotion, I change the way I'm thinking about the situation. \\
SP -01 & I keep my emotions to myself. \\
WP -02 & I control my emotions by not expressing them. \\
SP -03 & When I am feeling negative emotions, I make sure not to express them. \\
SP -04 & Whenever I am faced with an undesired emotion, I try to take my mind off it by thinking about something irrelevant. \\
DS $-\mathbf{0 1}$ & I distract myself from emotions that I do not want to feel. \\
DS $-\mathbf{0 2}$ & Whenever I am frustrated, I try to think about something else in order to let myself cool off. \\
DS $-\mathbf{0 3}$ & I control the emotions I am feeling by distracting myself from them. \\
DS $-\mathbf{0 4}$ & If something is upsetting me, I try to think about something else \\
DS $-\mathbf{0 5}$ & Focusing on the boring parts of emotional experiences helps me get past unwanted feelings. \\
SA $-\mathbf{0 1}$ & I manage my emotions by focusing on the unemotional parts of an experience. \\
SA $-\mathbf{0 2}$ & I try to focus on the neutral parts of intense experiences in order to control my emotions. \\
SA $-\mathbf{0 3}$ & I focus on the mundane aspects of emotional experiences if I do not want to feel my emotions strongly \\
SA $-\mathbf{0 4}$ & I control my emotions by physically changing the situation I'm in. \\
SS $-\mathbf{0 1}$ & When I want to feel a positive emotion, I try to physically put myself in situations or places that I know make me feel good. \\
SS $-\mathbf{0 2}$ & If I do not like how I am feeling, I try to change my surroundings. \\
SS $-\mathbf{0 3}$ &
\end{tabular}

Note. 'RP' refers to Reappraisal items; 'SP' refers to Suppression items; 'DS' refers to Distraction items; 'SA' refers to Selective Attention items; 'SS' refers to Situation Selection items. Reappraisal and Suppression items were originally developed by Gross \& John (2003). Labels for novel items (DS, SA, SS) are in bold. Participants were administered a random ordering of all items (i.e., a single randomized ordering was given to all participants). The items for Distraction, Selective Attention, and Situation Selection are the final items, pared down from a broader pool of items. Discarded items can be found on the OSF (osf.io/d2hku). 
Supplementary Table 2. Parameter estimates of exploratory IRT analyses from the first iteration of the E-ERQ (ONLINE1)

\begin{tabular}{|c|c|c|c|c|c|c|c|}
\hline Strategy - Item & $a$ & $b 1$ & $b 2$ & $b 3$ & $b 4$ & $b 5$ & $b 7$ \\
\hline $\mathrm{RP}-01$ & $3.2[2.7,3.7]$ & $-2.3[-2.6,-1.9]$ & $-1.6[-1.9,1.4]$ & $-1.2[-1.4,-1.0]$ & $-0.8[-0.9,-0.6]$ & $0.0[-0.1,0.2]$ & $1.1[0.9,1.2]$ \\
\hline $\mathrm{RP}-02$ & $2.6[2.2,3.0]$ & $-2.7[-3.2,-2.2]$ & $-1.9[-2.1,-1.6]$ & $-1.4[-1.7,-1.2]$ & $-0.8[-0.9,-0.6]$ & $0.1[-0.0,0.2]$ & $1.1[0.9,1.3]$ \\
\hline $\mathrm{RP}-03$ & $2.7[2.2,3.1]$ & $-2.5[-2.8,-2.1]$ & $-1.8[-2.1,-1.6]$ & $-1.4[-1.6,-1.2]$ & $-0.8[-1.0,-0.7]$ & $0.1[-0.1,0.2]$ & $1.1[0.1,1.3]$ \\
\hline$R P-04$ & $2.7[2.3,3.1]$ & $-2.1[-2.4,-1.8]$ & $-1.7[-1.9,-1.5]$ & $-1.3[-1.5,-1.1]$ & $-0.7[-0.9,-0.6]$ & $0.2[0.1,0.4]$ & $1.2[1.0,1.4]$ \\
\hline $\mathrm{RP}-05$ & $3.9[3.2,4.5]$ & $-2.1[-2.4,-1.8]$ & $-1.5[-1.7,-1.3]$ & $-1.1[-1.3,-0.9]$ & $-0.7[-0.8,-0.5]$ & $0.1[0.0,0.3]$ & $1.0[0.8,1.2]$ \\
\hline$R P-06$ & $3.4[2.9,4.0]$ & $-2.3[-2.6,-1.9]$ & $-1.6[-1.8,-1.4]$ & $-1.1[-1.3,-1.0]$ & $-0.7[-0.9,-0.6]$ & $0.2[0.0,0.3]$ & $1.3[0.9,1.3]$ \\
\hline $\mathrm{SP}-01$ & $2.5[2.0,3.0]$ & $-1.9[-2.2,-1.6]$ & $-1.2[-1.4,-1.0]$ & $-0.6[-0.8,-0.4]$ & $-0.1[-0.2,0.1]$ & $0.6[0.5,0.8]$ & $1.5[1.2,1.7]$ \\
\hline $\mathrm{SP}-02$ & $2.6[2.1,3.1]$ & $-1.9[-2.2,-1.7]$ & $-1.1[-1.3,-0.9]$ & $-0.4[-0.5,-0.2]$ & $0.1[-0.0,0.2]$ & $0.8[0.6,0.9]$ & $1.5[1.3,1.8]$ \\
\hline $\mathrm{SP}-03$ & $2.6[2.1,3.1]$ & $-1.5[-1.8,-1.3]$ & $-0.9[-1.1,-0.7]$ & $-0.3[-0.4,-0.1]$ & $0.3[0.1,0.4]$ & $0.9[0.7,1.1]$ & $1.9[1.6,2.2]$ \\
\hline $\mathrm{SP}-04$ & $1.4[1.1,1.7]$ & $-1.1[-1.4,-0.9]$ & $-0.1[-0.3,0.1]$ & $0.5[0.3,0.7]$ & $1.1[0.8,1.3]$ & $1.8[1.4,2.2]$ & $2.7[2.1,3.2]$ \\
\hline $\mathrm{DS}-01$ & $0.4[0.2,0.6]$ & $-7.2[-10.5,-3.9]$ & $-4.5[-6.6,-2.5]$ & $-1.9[-2.8,-0.9]$ & $-0.7[-1.3,-0.2]$ & $1.3[0.6,2.1]$ & $4.3[2.3,6.3]$ \\
\hline $\mathrm{DS}-02$ & $1.6[1.3,1.9]$ & $-3.0[-3.6,-2.4]$ & $-2.2[-2.6,-1.8]$ & $-1.6[-1.9,-1.3]$ & $-1.0[-1.3,-0.8]$ & $0.1[-0.1,0.2]$ & $1.4[1.1,1.7]$ \\
\hline $\mathrm{DS}-03$ & $0.2[0.1,0.4]$ & $-4.8[-8.8,-0.9]$ & $-1.0[-2.2,0.1]$ & $2.0[0.1,3.8]$ & $4.9[0.8,9.0]$ & $9.7[1.8,17.6]$ & $18.9[3.2,34.7]$ \\
\hline $\mathrm{DS}-04$ & $0.4[0.2,0.6]$ & $-7.3[-11.0,-3.5]$ & $-4.0[-6.1,-2.0]$ & $-1.4[-2.3,-0.6]$ & $1.2[0.4,2.1]$ & $3.8[1.8,5.8]$ & $7.2[3.5,10.9]$ \\
\hline $\mathrm{DS}-05$ & $0.9[0.7,1.1]$ & $-2.9[-3.7,-2.2]$ & $-1.9[-2.4,-1.4]$ & $-1.3[-1.6,-0.9]$ & $-0.4[-0.7,-0.1]$ & $0.9[0.5,1.2]$ & $2.4[1.8,3.0]$ \\
\hline $\mathrm{DS}-06$ & $1.7[1.4,2.0]$ & $-2.6[-3.1,-2.1]$ & $-1.6[-1.9,-1.3]$ & $-0.9[-1.1,-0.7]$ & $-0.2[-0.4,-0.0]$ & $0.8[0.6,1.0]$ & $2.1[1.8,2.5]$ \\
\hline $\mathrm{DS}-07$ & $1.9[1.6,2.3]$ & $-2.5[-2.9,-2.0]$ & $-1.8[-2.1,-1.5]$ & $-1.2[-1.4,-1.0]$ & $-0.6[-0.8,-0.4]$ & $0.6[0.4,0.7]$ & $1.7[1.4,2.0]$ \\
\hline $\mathrm{DS}-08$ & $1.3[1.0,1.5]$ & $-3.1[-3.8,-2.5]$ & $-2.3[-2.8,-1.8]$ & $-1.8[-2.2,-1.4]$ & $-1.3[-1.6,-1.0]$ & $-0.0[-0.2,0.2]$ & $1.4[1.1,1.7]$ \\
\hline DS - 09 & $1.2[0.9,1.4]$ & $-3.2[-3.9,-2.5]$ & $-1.8[-2.2,-1.4]$ & $-0.9[-1.2,-0.6]$ & $-0.3[-0.5,-0.1]$ & $1.0[0.7,1.2]$ & $2.4[1.9,2.9]$ \\
\hline $\mathrm{DS}-10$ & $2.3[1.9,2.7]$ & $-2.4[-2.8,-2.0]$ & $-1.6[-1.9,-1.4]$ & $-1.3[-1.5,-1.1]$ & $-0.7[-0.9,-0.6]$ & $0.3[0.1,0.4]$ & $1.5[1.2,1.7]$ \\
\hline $\mathrm{DS}-11$ & $2.8[2.3,3.2]$ & $-2.7[-3.2,-2.2]$ & $-1.8[-2.0,-1.5]$ & $-1.4[-1.6,-1.2]$ & $-0.9[-1.1,-0.7]$ & $0.2[0.1,0.4]$ & $1.3[1.1,1.5]$ \\
\hline $\mathrm{DS}-12$ & $2.0[1.6,2.3]$ & $-3.0[-3.5,-2.4]$ & $-1.7[-2.0,-1.4]$ & $-1.2[-1.4,-0.9]$ & $-0.6[-0.7,-0.4]$ & $0.5[0.4,0.7]$ & $1.6[1.4,1.9]$ \\
\hline $\mathrm{DS}-13$ & $1.9[1.5,2.2]$ & $-2.7[-3.2,-2.2]$ & $-1.8[-2.1,-1.5]$ & $-1.3[-1.5,-1.1]$ & $-0.8[-1.0,-0.6]$ & $0.4[0.2,0.5]$ & $1.6[1.3,1.9]$ \\
\hline $\mathrm{DS}-14$ & $2.4[2.0,2.9]$ & $-2.7[-3.2,-2.2]$ & $-2.2[-2.5,-1.8]$ & $-1.6[-1.8,-1.3]$ & $-1.0[-1.2,-0.8]$ & $-0.0[-0.2,0.1]$ & $1.1[0.9,1.3]$ \\
\hline $\mathrm{SA}-01$ & $1.1[0.9,1.4]$ & $-3.5[-4.3,-2.7]$ & $-2.6[-3.1,-2.0]$ & $-1.8[-2.2,-1.4]$ & $-0.7[-0.9,-0.4]$ & $0.9[0.6,1.2]$ & $2.6[2.0,3.1]$ \\
\hline $\mathrm{SA}-02$ & $1.3[1.1,1.6]$ & $-1.3[-1.6,-1.0]$ & $-0.2[-0.4,0.0]$ & $0.4[0.2,0.6]$ & $1.2[0.9,1.5]$ & $2.6[2.1,3.2]$ & $3.7[2.9,4.6]$ \\
\hline $\mathrm{SA}-03$ & $1.8[1.5,2.1]$ & $-1.7[-2.0,-1.4]$ & $-0.8[-1.0,-0.6]$ & $-0.3[-0.5,-0.2]$ & $0.3[0.1,0.4]$ & $1.3[1.1,1.6]$ & $2.2[1.8,2.6]$ \\
\hline $\mathrm{SA}-04$ & $1.7[1.4,2.0]$ & $-2.2[-2.6,-1.8]$ & $-1.2[-1.5,-1.0]$ & $-0.5[-0.7,-0.3]$ & $0.2[0.0,0.3]$ & $1.3[1.0,1.5]$ & $2.3[1.9,2.7]$ \\
\hline $\mathrm{SA}-05$ & $2.2[1.9,2.6]$ & $-1.3[-1.6,-1.1]$ & $-0.5[-0.7,-0.3]$ & $-0.1[-0.2,0.1]$ & $0.4[0.3,0.6]$ & $1.3[1.0,1.5]$ & $2.2[1.8,2.5]$ \\
\hline $\mathrm{SA}-06$ & $1.3[1.1,1.6]$ & $-3.5[-4.3,-2.8]$ & $-2.3[-2.8,-1.8]$ & $-1.6[-1.9,-1.3]$ & $-0.8[-1.1,-0.6]$ & $0.5[0.3,0.7]$ & $1.9[1.5,2.3]$ \\
\hline$S A-07$ & $1.4[1.2,1.7]$ & $-2.1[-2.5,-1.7]$ & $-1.2[-1.5,-0.9]$ & $-0.4[-0.6,-0.2]$ & $0.3[0.1,0.5]$ & $1.4[1.1,1.7]$ & $2.4[2.0,2.9]$ \\
\hline
\end{tabular}




\begin{tabular}{|c|c|c|c|c|c|c|c|}
\hline $\mathrm{SA}-08$ & $-0.4[-0.6,-0.2]$ & $6.0[2.9,9.1]$ & $3.3[1.6,4.9]$ & $0.2[-0.3,0.7]$ & $-2.0[-3.1,-0.9]$ & $-4.3[-6.5,-2.0]$ & $-6.6[-10.0,-3.2]$ \\
\hline $\mathrm{SA}-09$ & $2.5[2.1,2.9]$ & $-1.5[-1.8,-1.3]$ & $-0.8[-0.9,-0.6]$ & $-0.2[-0.4,-0.1]$ & $0.5[0.3,0.6]$ & $1.3[1.0,1.5]$ & $2.2[1.8,2.6]$ \\
\hline $\mathrm{SA}-10$ & $-0.5[-0.7,-0.3]$ & $5.8[3.1,8.5]$ & $2.9[1.5,4.2]$ & $0.4[0.0,0.9]$ & $-1.4[-2.2,-0.6]$ & $-3.5[-5.2,-1.8]$ & $-6.3[-9.2,-3.4]$ \\
\hline $\mathrm{SA}-11$ & $2.5[2.1,2.9]$ & $-1.7[-2.0,-1.4]$ & $-0.8[-1.0,-0.6]$ & $-0.3[-0.4,-0.1]$ & $0.4[0.2,0.5]$ & $1.2[1.0,1.5]$ & $2.1[1.8,2.4]$ \\
\hline $\mathrm{SA}-12$ & $2.5[2.1,2.9]$ & $-1.8[-2.1,-1.6]$ & $-0.9[-1.1,-0.8]$ & $-0.4[-0.5,-0.2]$ & $0.3[0.1,0.4]$ & $1.1[0.9,1.3]$ & $1.9[1.7,2.2]$ \\
\hline $\mathrm{SA}-13$ & $1.1[0.9,1.3]$ & $-2.9[-3.5,-2.3]$ & $-1.6[-2.0,-1.2]$ & $-0.8[-1.1,-0.5]$ & $0.0[-0.2,0.3]$ & $1.1[0.8,1.4]$ & $2.4[1.9,2.9]$ \\
\hline $\mathrm{SA}-14$ & $3.0[2.6,3.5]$ & $-1.5[-1.7,-1.3]$ & $-0.9[-1.0,-0.7]$ & $-0.3[-0.5,-0.2]$ & $0.3[0.2,0.4]$ & $1.1[0.9,1.2]$ & $2.0[1.7,2.3]$ \\
\hline $\mathrm{SS}-01$ & $1.3[1.0,1.6]$ & $-3.2[-3.9,-2.5]$ & $-2.3[-2.7,-1.8]$ & $-1.3[-1.6,-1.0]$ & $-0.5[-0.7,-0.3]$ & $0.8[0.6,1.1]$ & $2.1[1.7,2.6]$ \\
\hline $\mathrm{SS}-02$ & $1.0[0.7,1.2]$ & $-3.3[-4.1,-2.5]$ & $-2.2[-2.7,-1.6]$ & $-1.4[-1.8,-1.0]$ & $-0.7[-1.0,-0.5]$ & $0.7[0.4,1.0]$ & $2.1[1.6,2.6]$ \\
\hline $\mathrm{SS}-03$ & $1.0[0.7,1.2]$ & $-3.6[-4.6,-2.7]$ & $-2.6[-3.3,-2.0]$ & $-2.0[-2.5,-1.5]$ & $-1.2[-1.6,-0.9]$ & $0.1[-0.1,0.3]$ & $1.8[1.3,2.2]$ \\
\hline $\mathrm{SS}-04$ & $0.2[0.0,0.4]$ & $-19.0[-35.3,-2.7]$ & $-10.4[-19.2,-1.7]$ & $-6.8[-12.5,-1.1]$ & $-3.3[-6.2,-0.5]$ & $-0.6[-1.5,0.3]$ & $5.0[0.7,9.3]$ \\
\hline$S S-05$ & $0.7[0.5,1.0]$ & $-4.5[-6.0,-3.0]$ & $-3.1[-4.1,-2.1]$ & $-2.0[-2.7,-1.3]$ & $-1.1[-1.6,-0.7]$ & $0.4[0.1,0.8]$ & $2.5[1.7,3.3]$ \\
\hline$S S-06$ & $2.2[1.8,2.6]$ & $-2.4[-2.8,-1.9]$ & $-1.9[-2.2,-1.6]$ & $-1.3[-1.5,-1.1]$ & $-0.7[-0.9,-0.5]$ & $0.3[0.1,0.4]$ & $1.3[1.1,1.6]$ \\
\hline$S S-07$ & $1.1[0.8,1.4]$ & $-3.7[-4.6,-2.8]$ & $-2.2[-2.8,-1.7]$ & $-1.3[-1.6,-1.0]$ & $-0.5[-0.7,-0.2]$ & $0.9[0.6,1.2]$ & $2.4[1.9,3.0]$ \\
\hline $\mathrm{SS}-08$ & $1.3[1.0,1.6]$ & $-3.3[-4.0,-2.5]$ & $-2.1[-2.5,-1.6]$ & $-1.5[-1.9,-1.2]$ & $-0.8[-1.0,-0.5]$ & $0.3[0.1,0.5]$ & $1.8[1.4,2.2]$ \\
\hline $\mathrm{SS}-09$ & $2.0[1.6,2.4]$ & $-2.7[-3.3,-2.2]$ & $-1.6[-1.9,-1.3]$ & $-1.0[-1.2,-0.8]$ & $-0.5[-0.7,-0.4]$ & $0.4[0.3,0.6]$ & $1.8[1.5,2.1]$ \\
\hline$S S-10$ & $1.4[1.1,1.7]$ & $-3.5[-4.3,-2.7]$ & $-2.9[-3.5,-2.3]$ & $-2.2[-2.6,-1.7]$ & $-1.3[-1.6,-1.0]$ & $-0.2[-0.4,0.0]$ & $1.3[1.0,1.6]$ \\
\hline $\mathrm{SS}-11$ & $1.4[1.1,1.7]$ & $-3.6[-4.4,-2.7]$ & $-2.7[-3.3,-2.1]$ & $-2.5[-3.0,-1.9]$ & $-1.8[-2.2,-1.4]$ & $-0.8[-1.0,-0.6]$ & $0.6[0.3,0.8]$ \\
\hline $\mathrm{SS}-12$ & $2.0[1.6,2.4]$ & $-2.4[-2.9,-2.0]$ & $-1.7[-2.0,-1.5]$ & $-1.3[-1.5,-1.1]$ & $-0.7[-0.8,-0.5]$ & $0.1[-0.1,0.2]$ & $1.1[0.9,1.3]$ \\
\hline
\end{tabular}

Note. ' $a$ ' refers to the discrimination parameter (the strength of association between the latent trait and a given item); ' $b$ ' refers to

location/difficulty parameter, indicating the position on the latent trait that corresponds with a .5 chance of rating an item at a particular scale value or a greater value. Brackets contain theoretical 95\% confidence intervals. All analyses implemented in R's MIRT package. 'RP' refers to Reappraisal items; 'SP' refers to Suppression items; 'DS' refers to Distraction items; 'SA' refers to Selective Attention items; 'SS' refers to Situation Selection items. Reappraisal and Suppression items were originally developed by Gross \& John (2003); IRT models were run on RP and SP items primarily for benchmarking purposes. The final items eventually selected for the three new strategies (DS, SA, SS) are displayed in green; items discarded after this round of validation are displayed in red; items that were not discarded this round, but discarded after the next round. DS, SA, and SS items here are numbered sequentially for convenience; item numbering changes across each of the three validation studies. Exploratory unidimensional IRT models were run for each strategy (i.e., this table reflects 5 separate IRT models). 
Supplementary Table 3. Parameter estimates of confirmatory IRT analyses from the second iteration of the E-ERQ (ONLINE2)

\begin{tabular}{|c|c|c|c|c|c|c|c|}
\hline Strategy - Item & $a$ & $b 1$ & $b 2$ & $b 3$ & $b 4$ & $b 5$ & $b 7$ \\
\hline $\mathrm{RP}-01$ & $2.5[2.1,2.8]$ & $-2.2[-2.6,-1.9]$ & $-1.7[-1.9,-1.4]$ & $-1.2[-1.4,-1.0]$ & $-0.7[-0.9,-0.6]$ & $0.2[0.0,0.3]$ & $1.1[0.9,1.3]$ \\
\hline$R P-02$ & $3.2[2.7,3.8]$ & $-2.3[-2.7,-2.0]$ & $-1.7[-1.9,-1.5]$ & $-1.2[-1.4,-1.1]$ & $-0.8[-1.0,-0.7]$ & $0.0[-0.2,0.1]$ & $0.9[0.7,1.1]$ \\
\hline$R P-03$ & $2.8[2.3,3.2]$ & $-2.4[-2.8,-2.1]$ & $-1.7[-1.9,-1.4]$ & $-1.3[-1.6,-1.1]$ & $-0.9[-1.0,-0.7]$ & $0.0[-0.1,0.2]$ & $1.1[0.9,1.3]$ \\
\hline$R P-04$ & $3.8[3.2,4.4]$ & $-2.0[-2.3,-1.7]$ & $-1.5[-1.7,-1.3]$ & $-1.1[-1.2,-0.9]$ & $-0.7[-0.8,-0.5]$ & $0.1[0.0,0.3]$ & $1.0[0.8,1.2]$ \\
\hline$R P-05$ & $4.7[3.9,5.4]$ & $-1.9[-2.1,-1.6]$ & $-1.4[-1.6,-1.2]$ & $-1.0[-1.2,-0.9]$ & $-0.6[-0.8,-0.5]$ & $0.1[-0.1,0.2]$ & $0.9[0.8,1.1]$ \\
\hline$R P-06$ & $4.6[3.9,5.4]$ & $-2.0[-2.2,-1.7]$ & $-1.5[-1.7,-1.3]$ & $-1.2[-1.3,-1.0]$ & $-0.8[-0.9,-0.7]$ & $0.0[-0.1,0.2]$ & $1.0[0.8,1.1]$ \\
\hline $\mathrm{SP}-01$ & $2.8[2.3,3.3]$ & $-1.5[-1.8,-1.3]$ & $-0.8[-1.0,-0.7]$ & $-0.4[-0.5,-0.3]$ & $0.1[-0.1,0.2]$ & $0.5[0.4,0.7]$ & $1.3[1.1,1.5]$ \\
\hline $\mathrm{SP}-02$ & $1.6[1.3,1.9]$ & $-0.8[-1.0,-0.6]$ & $0.1[-0.1,0.3]$ & $0.7[0.5,1.0]$ & $1.1[0.9,1.4]$ & $1.8[1.5,2.1]$ & $2.5[2.0,3.0]$ \\
\hline $\mathrm{SP}-03$ & $4.9[3.5,6.3]$ & $-1.1[-1.2,-0.9]$ & $-0.5[-0.7,-0.4]$ & $-0.1[-0.3,0.0]$ & $0.2[0.1,0.3]$ & $0.7[0.6,0.9]$ & $1.3[1.1,1.5]$ \\
\hline $\mathrm{SP}-04$ & $2.8[2.3,3.3]$ & $-1.4[-1.7,-1.2]$ & $-0.9[-1.0,-0.7]$ & $-0.3[-0.4,-0.1]$ & $0.2[0.0,0.3]$ & $0.7[0.6,0.9]$ & $1.5[1.3,1.7]$ \\
\hline $\mathrm{DS}-01$ & $1.8[1.5,2.1]$ & $-2.8[-3.3,-2.2]$ & $-2.0[-2.3,-1.6]$ & $-1.5[-1.8,-1.2]$ & $-0.9[-1.1,-0.7]$ & $0.3[0.1,0.4]$ & $1.4[1.2,1.7]$ \\
\hline $\mathrm{DS}-02$ & $2.1[1.7,2.4]$ & $-2.2[-2.6,-1.9]$ & $-1.5[-1.8,-1.3]$ & $-1.1[-1.3,-0.8]$ & $-0.6[-0.7,-0.4]$ & $0.5[0.4,0.7]$ & $1.6[1.3,1.8]$ \\
\hline $\mathrm{DS}-03$ & $2.6[2.1,3.0]$ & $-2.0[-2.3,-1.7]$ & $-1.4[-1.7,-1.2]$ & $-1.1[-1.3,-0.9]$ & $-0.6[-0.8,-0.5]$ & $0.4[0.3,0.6]$ & $1.4[1.2,1.6]$ \\
\hline $\mathrm{DS}-04$ & $2.6[2.2,3.1]$ & $-2.2[-2.6,-1.9]$ & $-1.6[-1.9,-1.4]$ & $-1.2[-1.4,-1.0]$ & $-0.8[-1.0,-0.6]$ & $0.1[0.0,0.3]$ & $1.3[1.1,1.5]$ \\
\hline $\mathrm{DS}-05$ & $2.1[1.8,2.5]$ & $-2.5[-2.9,-2.1]$ & $-2.0[-2.3,-1.7]$ & $-1.5[-1.7,-1.3]$ & $-1.0[-1.2,-0.8]$ & $0.3[0.2,0.5]$ & $1.2[1.0,1.5]$ \\
\hline $\mathrm{DS}-06$ & $2.5[2.1,2.9]$ & $-2.1[-2.4,-1.8]$ & $-1.6[-1.8,-1.4]$ & $-1.1[-1.3,-0.9]$ & $-0.7[-0.9,-0.6]$ & $0.3[0.2,0.5]$ & $1.3[1.1,1.5]$ \\
\hline $\mathrm{DS}-07$ & $1.5[1.3,1.8]$ & $-2.8[-3.3,-2.2]$ & $-2.0[-2.3,-1.6]$ & $-1.5[-1.8,-1.2]$ & $-0.9[-1.2,-0.7]$ & $0.3[0.1,0.5]$ & $1.5[1.2,1.8]$ \\
\hline $\mathrm{DS}-08$ & $2.4[2.0,2.8]$ & $-2.7[-3.1,-2.2]$ & $-2.0[-2.3,-1.7]$ & $-1.5[-1.7,-1.3]$ & $-1.0[-1.2,-0.8]$ & $0.0[-0.2,0.1]$ & $1.2[1.0,1.4]$ \\
\hline $\mathrm{SA}-01$ & $1.5[1.2,1.7]$ & $-2.0[-2.3,-1.6]$ & $-1.0[-1.3,-0.8]$ & $-0.5[-0.7,-0.3]$ & $0.4[0.2,0.6]$ & $1.2[1.0,1.5]$ & $2.1[1.7,2.5]$ \\
\hline $\mathrm{SA}-02$ & $1.4[1.1,1.7]$ & $-1.4[-1.7,-1.1]$ & $-0.4[-0.6,-0.2]$ & $0.2[0.0,0.4]$ & $0.7[0.5,0.9]$ & $1.5[1.2,1.8]$ & $2.4[1.9,2.8]$ \\
\hline $\mathrm{SA}-03$ & $2.6[2.1,3.0]$ & $-1.4[-1.6,-1.1]$ & $-0.6[-0.7,-0.4]$ & $0.0[-0.2,0.1]$ & $0.4[0.2,0.5]$ & $1.2[1.0,1.4]$ & $2.1[1.7,2.4]$ \\
\hline $\mathrm{SA}-04$ & $2.8[2.3,3.3]$ & $-1.6[-1.9,-1.4]$ & $-0.8[-0.9,-0.6]$ & $-0.3[-0.4,-0.2]$ & $0.3[0.1,0.4]$ & $1.0[0.8,1.2]$ & $2.0[1.7,2.3]$ \\
\hline $\mathrm{SA}-05$ & $2.9[2.4,3.4]$ & $-1.5[-1.7,-1.3]$ & $-0.8[-1.0,-0.6]$ & $-0.3[-0.5,-0.2]$ & $0.4[0.2,0.5]$ & $1.1[1.0,1.3]$ & $1.8[1.6,2.1]$ \\
\hline $\mathrm{SA}-06$ & $2.8[2.3,3.3]$ & $-1.4[-1.7,-1.2]$ & $-0.7[-0.9,-0.6]$ & $-0.1[-0.3,0.0]$ & $0.4[0.3,0.5]$ & $1.1[0.9,1.3]$ & $1.8[1.5,2.1]$ \\
\hline $\mathrm{SS}-01$ & $2.6[2.2,3.1]$ & $-2.0[-2.3,-1.7]$ & $-1.3[-1.5,-1.1]$ & $-0.8[-1.0,-0.7]$ & $-0.3[-0.5,-0.2]$ & $0.7[0.5,0.8]$ & $1.6[1.4,1.9]$ \\
\hline$S S-02$ & $4.4[3.3,5.4]$ & $-1.8[-2.1,-1.6]$ & $-1.4[-1.6,-1.2]$ & $-1.0[-1.2,-0.8]$ & $-0.6[-0.7,-0.4]$ & $0.2[0.1,0.3]$ & $1.1[0.9,1.3]$ \\
\hline $\mathrm{SS}-03$ & $2.9[2.4,3.4]$ & $-2.2[-2.5,-1.8]$ & $-1.5[-1.7,-1.3]$ & $-1.0[-1.2,-0.8]$ & $-0.5[-0.7,-0.4]$ & $0.3[0.2,0.5]$ & $1.3[1.1,1.6]$ \\
\hline$S S-04$ & $0.9[0.6,1.1]$ & $-5.7[-7.5,-3.9]$ & $-4.9[-6.3,-3.4]$ & $-3.3[-4.2,-2.4]$ & $-2.2[-2.8,-1.6]$ & $-0.4[-0.7,-0.1]$ & $1.4[0.9,1.8]$ \\
\hline$S S-05$ & $1.2[0.9,1.4]$ & $-3.9[-4.8,-2.9]$ & $-3.2[-4.0,-2.5]$ & $-2.6[-3.2,-2.0]$ & $-2.0[-2.5,-1.5]$ & $-0.8[-1.1,-0.5]$ & $0.8[0.5,1.0]$ \\
\hline $\mathrm{SS}-06$ & $1.4[1.1,1.6]$ & $-3.1[-3.8,-2.5]$ & $-2.1[-2.5,-1.7]$ & $-1.4[-1.7,-1.1]$ & $-0.8[-1,-0.6]$ & $0.4[0.2,0.6]$ & $1.7[1.4,2.1]$ \\
\hline
\end{tabular}




\section{EMOTION REGULATION TYPES}

Note. ' $a$ ' refers to the discrimination parameter (the strength of association between the latent trait and a given item); ' $b$ ' refers to location/difficulty parameter, indicating the position on the latent trait that corresponds with a .5 chance of rating an item at a particular scale value or a greater value. Brackets contain theoretical 95\% confidence intervals. All analyses implemented in R's MIRT package. 'RP' refers to Reappraisal items; 'SP' refers to Suppression items; 'DS' refers to Distraction items; 'SA' refers to Selective Attention items; 'SS' refers to Situation Selection items. Reappraisal and Suppression items were originally developed by Gross \& John (2003); IRT models were run on RP and SP items primarily for benchmarking purposes. The final items eventually selected for the three new strategies (DS, SA, SS) are displayed in green; items discarded after this round of validation are displayed in red. DS, SA, and SS items here are numbered sequentially for convenience; item numbering changes across each of the three validation studies. Confirmatory unidimensional IRT models were run for each strategy (i.e., this table reflects 5 separate IRT models). 


\begin{tabular}{|c|c|c|c|c|c|c|c|}
\hline Strategy - Item & $a$ & $b 1$ & $b 2$ & $b 3$ & $b 4$ & $b 5$ & $b 7$ \\
\hline $\mathrm{RP}-01$ & $1.2[0.9,1.5]$ & $-3.8[-4.8,-2.8]$ & $-2.7[-3.4,-2.0]$ & $-1.9[-2.4,-1.4]$ & $-0.9[-1.2,-0.6]$ & $0.5[0.2,0.7]$ & $2.1[1.6,2.6]$ \\
\hline $\mathrm{RP}-02$ & $1.7[1.4,2.1]$ & $-2.7[-3.3,-2.2]$ & $-1.8[-2.2,-1.5]$ & $-1.1[-1.4,-0.8]$ & $-0.5[-0.7,-0.3]$ & $0.6[0.4,0.8]$ & $1.7[1.3,2.0]$ \\
\hline$R P-03$ & $1.4[1.1,1.7]$ & $-2.7[-3.3,-2.2]$ & $-1.8[-2.2,-1.4]$ & $-1.2[-1.5,-0.9]$ & $-0.7[-0.9,-0.4]$ & $0.7[0.4,0.9]$ & $1.9[1.5,2.3]$ \\
\hline$R P-04$ & $3.3[2.6,4.0]$ & $-2.4[-2.8,-1.9]$ & $-1.5[-1.7,-1.2]$ & $-0.8[-1.0,-0.6]$ & $-0.5[-0.7,-0.3]$ & $0.5[0.3,0.6]$ & $1.5[1.2,1.7]$ \\
\hline$R P-05$ & $3.1[2.5,3.7]$ & $-2.4[-2.8,-2.0]$ & $-1.6[-1.9,-1.3]$ & $-1.0[-1.2,-0.8]$ & $-0.6[-0.7,-0.4]$ & $0.5[0.3,0.6]$ & $1.6[1.3,1.8]$ \\
\hline$R P-06$ & $3.6[2.8,4.3]$ & $-2.2[-2.6,-1.8]$ & $-1.5[-1.7,-1.2]$ & $-0.9[-1.1,-0.7]$ & $-0.4[-0.6,-0.2]$ & $0.6[0.4,0.8]$ & $1.5[1.2,1.7]$ \\
\hline $\mathrm{SP}-01$ & $2.0[1.5,2.5]$ & $-2.4[-2.9,-1.9]$ & $-1.4[-1.7,-1.1]$ & $-0.7[-0.9,-0.5]$ & $-0.2[-0.4,0.0]$ & $0.3[0.1,0.5]$ & $1.2[0.9,1.4]$ \\
\hline $\mathrm{SP}-02$ & $0.9[0.7,1.2]$ & $-1.4[-1.9,-1.0]$ & $0.2[-0.1,0.5]$ & $1.3[0.9,1.7]$ & $2.0[1.4,2.6]$ & $3.5[2.5,4.5]$ & $4.7[3.2,6.3]$ \\
\hline $\mathrm{SP}-03$ & $3.4[2.1,4.7]$ & $-1.2[-1.4,-1.0]$ & $-0.5[-0.7,-0.3]$ & $-0.1[-0.2,0.1]$ & $0.3[0.2,0.5]$ & $0.9[0.7,1.1]$ & $1.8[1.4,2.1]$ \\
\hline $\mathrm{SP}-04$ & $1.5[1.2,1.9]$ & $-2.2[-2.7,-1.7]$ & $-1.0[-1.3,-0.8]$ & $-0.1[-0.3,0.1]$ & $0.3[0.1,0.5]$ & $1.1[0.8,1.4]$ & $2.1[1.6,2.6]$ \\
\hline $\mathrm{DS}-01$ & $1.9[1.5,2.3]$ & $-2.8[-3.3,-2.2]$ & $-1.5[-1.8,-1.2]$ & $-0.7[-0.9,-0.5]$ & $-0.2[-0.4,0.0]$ & $0.8[0.6,1.0]$ & $1.8[1.4,2.1]$ \\
\hline $\mathrm{DS}-02$ & $1.9[1.5,2.4]$ & $-2.3[-2.7,-1.8]$ & $-1.6[-1.9,-1.3]$ & $-0.9[-1.2,-0.7]$ & $-0.4[-0.6,-0.2]$ & $0.6[0.4,0.9]$ & $1.5[1.1,1.8]$ \\
\hline $\mathrm{DS}-03$ & $2.1[1.6,2.5]$ & $-2.6[-3.1,-2.0]$ & $-1.5[-1.8,-1.2]$ & $-0.9[-1.1,-0.7]$ & $-0.5[-0.7,-0.3]$ & $0.8[0.6,1.0]$ & $1.9[1.5,2.3]$ \\
\hline $\mathrm{DS}-04$ & $2.0[1.6,2.5]$ & $-2.5[-3.0,-2.0]$ & $-1.7[-2.0,-1.4]$ & $-1.0[-1.2,-0.8]$ & $-0.5[-0.7,-0.3]$ & $0.7[0.5,0.9]$ & $1.9[1.5,2.2]$ \\
\hline $\mathrm{DS}-05$ & $2.7[2.1,3.3]$ & $-2.5[-2.9,-2.0]$ & $-1.5[-1.8,-1.2]$ & $-0.8[-1.0,-0.6]$ & $-0.5[-0.7,-0.3]$ & $0.6[0.4,0.8]$ & $1.7[1.4,2.0]$ \\
\hline $\mathrm{SA}-01$ & $1.6[1.3,2.0]$ & $-1.6[-1.9,-1.2]$ & $-0.4[-0.6,-0.2]$ & $0.3[0.1,0.5]$ & $1.2[0.9,1.5]$ & $2.1[1.7,2.5]$ & $3.3[2.5,4.0]$ \\
\hline $\mathrm{SA}-02$ & $1.9[1.5,2.3]$ & $-1.5[-1.8,-1.2]$ & $-0.5[-0.7,-0.3]$ & $0.1[-0.1,0.3]$ & $0.8[0.6,1.0]$ & $1.6[1.3,2.0]$ & $2.7[2.1,3.3]$ \\
\hline$S A-03$ & $2.8[2.1,3.4]$ & $-1.7[-2.0,-1.4]$ & $-0.7[-0.9,-0.5]$ & $0.0[-0.1,0.2]$ & $0.6[0.4,0.8]$ & $1.5[1.3,1.8]$ & $2.6[2.1,3.1]$ \\
\hline$S A-04$ & $3.0[2.3,3.8]$ & $-1.5[-1.8,-1.2]$ & $-0.7[-0.9,-0.5]$ & $0.0[-0.2,0.1]$ & $0.6[0.5,0.8]$ & $1.6[1.3,1.9]$ & $2.2[1.8,2.6]$ \\
\hline $\mathrm{SS}-01$ & $2.4[1.8,2.9]$ & $-2.3[-2.8,-1.9]$ & $-1.4[-1.6,-1.1]$ & $-0.8[-1.0,-0.6]$ & $-0.3[-0.4,-0.1]$ & $0.8[0.6,1.0]$ & $1.8[1.5,2.1]$ \\
\hline $\mathrm{SS}-02$ & $2.9[2.1,3.6]$ & $-2.7[-3.3,-2.2]$ & $-1.8[-2.2,-1.5]$ & $-1.1[-1.4,-0.9]$ & $-0.8[-1.0,-0.6]$ & $0.2[0.0,0.3]$ & $1.2[0.9,1.4]$ \\
\hline $\mathrm{SS}-03$ & $2.5[1.9,3.1]$ & $-2.4[-2.9,-2.0]$ & $-1.7[-2.0,-1.4]$ & $-0.9[-1.1,-0.7]$ & $-0.6[-0.8,-0.4]$ & $0.7[0.5,0.9]$ & $1.7[1.4,2.0]$ \\
\hline
\end{tabular}

Note. ' $a$ ' refers to the discrimination parameter (the strength of association between the latent trait and a given item); ' $b$ ' refers to

location/difficulty parameter, indicating the position on the latent trait that corresponds with a .5 chance of rating an item at a particular scale value or a greater value. Brackets contain theoretical 95\% confidence intervals. All analyses implemented in R's MIRT package. 'RP' refers to

Reappraisal items; 'SP' refers to Suppression items; 'DS' refers to Distraction items; 'SA' refers to Selective Attention items; 'SS' refers to Situation Selection items. Reappraisal and Suppression items were originally developed by Gross \& John (2003); IRT models were run on RP and SP items primarily for benchmarking purposes. These are the final items for DS, SA, and SS, and their numbering matches that in Supplementary Table 1. Confirmatory unidimensional IRT models were run for each strategy (i.e., this table reflects 5 separate IRT models).

Supplementary Table 5. Factor loadings of the bi-factor model across all three validation samples 


\begin{tabular}{|c|c|c|c|c|c|c|}
\hline \multirow[b]{2}{*}{ Item } & \multicolumn{2}{|c|}{ ONLINE1 } & \multicolumn{2}{|c|}{ ONLINE2 } & \multicolumn{2}{|c|}{ ONLINE3 } \\
\hline & $\begin{array}{l}\text { General Factor } \\
\text { Loadings }\end{array}$ & $\begin{array}{c}\text { Strategy Specific Factor } \\
\text { Loadings }\end{array}$ & $\begin{array}{c}\text { General Factor } \\
\text { Loadings }\end{array}$ & $\begin{array}{c}\text { Strategy Specific Factor } \\
\text { Loadings }\end{array}$ & $\begin{array}{c}\text { General Factor } \\
\text { Loadings }\end{array}$ & $\begin{array}{l}\text { Strategy Specific } \\
\text { Factor Loadings }\end{array}$ \\
\hline $\mathrm{RP}-01$ & $0.737[0.643,0.831]$ & $0.311[0.195,0.428]$ & $0.777[0.689,0.865]$ & $0.089[-0.053,0.232]$ & $0.660[0.549,0.770]$ & $0.029[-0.108,0.167]$ \\
\hline $\mathrm{RP}-02$ & $0.794[0.707,0.881]$ & $0.025[-0.097,0.147]$ & $0.855[0.770,0.939]$ & $0.096[-0.051,0.243]$ & $0.822[0.718,0.926]$ & $0.059[-0.084,0.202]$ \\
\hline $\mathrm{RP}-03$ & $0.706[0.609,0.804]$ & $0.351[0.235,0.468]$ & $0.729[0.630,0.827]$ & $0.328[0.195,0.461]$ & $0.427[0.300,0.554]$ & $0.367[0.238,0.495]$ \\
\hline $\mathrm{RP}-04$ & $0.699[0.603,0.795]$ & $0.311[0.194,0.428]$ & $0.755[0.647,0.863]$ & $0.478[0.348,0.608]$ & $0.513[0.366,0.660]$ & $0.728[0.610,0.847]$ \\
\hline $\mathrm{RP}-05$ & $0.772[0.678,0.866]$ & $0.351[0.235,0.466]$ & $0.781[0.679,0.882]$ & $0.416[0.285,0.547]$ & $0.629[0.501,0.756]$ & $0.515[0.390,0.639]$ \\
\hline$R P-06$ & $0.725[0.622,0.828]$ & $0.474[0.356,0.592]$ & $0.796[0.697,0.895]$ & $0.390[0.258,0.522]$ & $0.600[0.464,0.737]$ & $0.621[0.499,0.743]$ \\
\hline $\mathrm{SP}-01$ & $-0.076[-0.188,0.035]$ & $0.755[0.664,0.845]$ & $-0.136[-0.244,-0.028]$ & $0.773[0.689,0.858]$ & $-0.014[-0.142,0.114]$ & $0.652[0.542,0.762]$ \\
\hline $\mathrm{SP}-02$ & $0.032[-0.081,0.144]$ & $0.783[0.694,0.873]$ & $-0.227[-0.332,-0.122]$ & $0.579[0.487,0.670]$ & $-0.220[-0.344,-0.095]$ & $0.461[0.346,0.575]$ \\
\hline $\mathrm{SP}-03$ & $-0.069[-0.181,0.043]$ & $0.777[0.687,0.866]$ & $-0.099[-0.209,0.011]$ & $0.897[0.817,0.976]$ & $-0.051[-0.182,0.080]$ & $0.881[0.777,0.984]$ \\
\hline$S P-04$ & $-0.253[-0.359,-0.147]$ & $0.544[0.448,0.640]$ & $0.015[-0.094,0.123]$ & $0.796[0.712,0.881]$ & $0.145[0.019,0.271]$ & $0.591[0.480,0.703]$ \\
\hline $\mathrm{DS}-01$ & $0.490[0.385,0.595]$ & $0.471[0.366,0.576]$ & $0.520[0.421,0.619]$ & $0.478[0.384,0.573]$ & $0.596[0.483,0.709]$ & $0.300[0.185,0.414]$ \\
\hline $\mathrm{DS}-02$ & $0.509[0.402,0.615]$ & $0.523[0.419,0.628]$ & $0.455[0.349,0.560]$ & $0.690[0.598,0.781]$ & $0.386[0.260,0.512]$ & $0.673[0.558,0.787]$ \\
\hline $\mathrm{DS}-03$ & $0.722[0.632,0.812]$ & $0.222[0.122,0.323]$ & $0.663[0.570,0.755]$ & $0.400[0.311,0.489]$ & $0.552[0.437,0.667]$ & $0.350[0.235,0.466]$ \\
\hline $\mathrm{DS}-04$ & $0.431[0.321,0.541]$ & $0.559[0.452,0.666]$ & $0.439[0.334,0.543]$ & $0.630[0.537,0.724]$ & $0.396[0.271,0.521]$ & $0.654[0.539,0.768]$ \\
\hline $\mathrm{DS}-05$ & $0.724[0.634,0.814]$ & $0.195[0.094,0.296]$ & $0.689[0.599,0.780]$ & $0.271[0.180,0.362]$ & $0.681[0.572,0.791]$ & $0.374[0.268,0.481]$ \\
\hline $\mathrm{SA}-01$ & $0.195[0.081,0.309]$ & $0.688[0.597,0.780]$ & $0.255[0.141,0.369]$ & $0.672[0.580,0.764]$ & $0.181[0.056,0.306]$ & $0.588[0.477,0.699]$ \\
\hline $\mathrm{SA}-02$ & $0.257[0.141,0.374]$ & $0.791[0.705,0.877]$ & $0.253[0.136,0.371]$ & $0.749[0.660,0.838]$ & $0.182[0.056,0.308]$ & $0.657[0.549,0.765]$ \\
\hline $\mathrm{SA}-03$ & $0.274[0.158,0.389]$ & $0.777[0.691,0.864]$ & $0.364[0.251,0.477]$ & $0.709[0.620,0.798]$ & $0.249[0.123,0.375]$ & $0.769[0.668,0.871]$ \\
\hline $\mathrm{SA}-04$ & $0.230[0.115,0.344]$ & $0.721[0.632,0.811]$ & $0.277[0.161,0.393]$ & $0.734[0.645,0.824]$ & $0.213[0.085,0.340]$ & $0.789[0.687,0.890]$ \\
\hline $\mathrm{SS}-01$ & $0.513[0.410,0.615]$ & $0.463[0.346,0.579]$ & $0.498[0.398,0.598]$ & $0.593[0.501,0.685]$ & $0.378[0.254,0.502]$ & $0.651[0.532,0.770]$ \\
\hline$S S-02$ & $0.543[0.440,0.646]$ & $0.541[0.425,0.658]$ & $0.629[0.534,0.725]$ & $0.629[0.544,0.715]$ & $0.500[0.381,0.619]$ & $0.611[0.498,0.725]$ \\
\hline $\mathrm{SS}-03$ & $0.532[0.432,0.633]$ & $0.419[0.303,0.534]$ & $0.526[0.427,0.625]$ & $0.599[0.508,0.690]$ & $0.476[0.356,0.596]$ & $0.600[0.485,0.714]$ \\
\hline \multicolumn{7}{|l|}{$\begin{array}{c}\text { Fit } \\
\text { Statistic }\end{array}$} \\
\hline$\chi^{2}(\mathrm{df})$ & \multicolumn{2}{|c|}{$492.368(177)$} & \multicolumn{2}{|c|}{$380.962(177)$} & \multicolumn{2}{|c|}{$272.776(177)$} \\
\hline CFI & \multicolumn{2}{|c|}{0.934} & \multicolumn{2}{|c|}{0.964} & \multicolumn{2}{|c|}{0.967} \\
\hline TLI & \multicolumn{2}{|c|}{0.914} & \multicolumn{2}{|c|}{0.953} & \multicolumn{2}{|c|}{0.957} \\
\hline $\begin{array}{c}\text { RMSE } \\
{[95 \% \mathrm{CI}]}\end{array}$ & \multicolumn{2}{|c|}{$0.067[0.060,0.074]$} & \multicolumn{2}{|c|}{$0.053[0.046,0.061]$} & \multicolumn{2}{|c|}{$0.043[0.032,0.052]$} \\
\hline
\end{tabular}

Note. ONLINE1 and ONLINE2 were collected via MTurk, ONLINE 3 was collected via an undergraduate student subject pool for online studies.

The same confirmatory bifactor model was fit on all three independent samples using R's 'lavaan' package. 'General Factor' refers to a factor reflecting trait-like propensity of emotion regulation usage; 'Strategy Specific' refers to group factors belonging to each specific strategy, reflecting common variance among items of a given strategy after partialing out the influence of general regulatory tendency. 'RP' refers to Reappraisal; 'SP' refers to Suppression; 'DS' refers to Distraction; 'SA' refers to Selective Attention; 'SS' refers to Situation Selection. Reappraisal and Suppression items were originally developed by Gross \& John (2003) 'CFI' refers to comparative fit index, 'TLI' refers to Tucker-Lewis Index, 'RMSEA' refers to root mean square error of approximation. 
67 EMOTION REGULATION TYPES

\begin{tabular}{c|ccccccc}
\hline Sample & $\omega$ & $\omega-\mathrm{h}$ & $\omega$-RP & $\omega$-SP & $\omega$-DS & $\omega$-SA & $\omega$-SS \\
\hline ONLINE1 & 0.926 & 0.717 & 0.910 & 0.914 & 0.910 & 0.914 & 0.909
\end{tabular}


68 EMOTION REGULATION TYPES

\begin{tabular}{c|llllllll} 
ONLINE2 & 0.942 & 0.717 & 0.927 & 0.931 & 0.929 & 0.930 & 0.927 \\
ONLINE3 & 0.908 & 0.640 & 0.883 & 0.884 & 0.883 & 0.886 & 0.880 \\
LAB1 & 0.904 & 0.530 & 0.865 & 0.865 & 0.863 & 0.865 & 0.857 \\
LAB2 & 0.855 & 0.170 & 0.746 & 0.645 & 0.684 & 0.659 & 0.640 \\
TEST-RETEST1 & 0.947 & 0.741 & 0.934 & 0.939 & 0.937 & 0.938 & 0.936 \\
TEST-RETEST2 & 0.946 & 0.719 & 0.931 & 0.935 & 0.934 & 0.937 & 0.933 \\
\hline
\end{tabular}

Supplementary Table 6. Model-based reliability estimates for the E-ERQ across 6 samples

Note. ONLINE1 and ONLINE2 were collected via MTurk, ONLINE 3 was collected via an undergraduate student subject pool for online studies, LAB 1 and LAB2 were laboratory studies whose participants were recruited from the UCLA undergraduate student community; TEST-RETEST was collected via MTurk, comprised of a pool of subjects who completed the measure on two separate occasions, approximately two-weeks apart (202 subjects participated at time 1,157 participated at time 2,156 participated in both). All estimates of reliability were obtained by fitting a confirmatory bifactor model on all E-ERQ items using R's 'lavaan' package. $\omega$ refers to the proportion of variance explained by loadings on all group factors plus the general factor; $\omega$-h refers to the proportion of variance explained by loadings on the general factor; all other $\omega$ 's refer to the proportion of variance explained by loadings on a given group factor plus the general factor. 'RP' refers to Reappraisal; 'SP' refers to Suppression; 'DS' refers to Distraction; 'SA' refers to Selective Attention; 'SS' refers to Situation Selection. Reappraisal and Suppression items were originally developed by Gross \& John (2003).

Supplementary Table 7. Test-Retest Reliability of the E-ERQ (TEST-RETEST)

\begin{tabular}{lr}
\hline Strategy & $r$ \\
\hline
\end{tabular}




\begin{tabular}{c|c}
\hline RP & 0.88 \\
SP & 0.86 \\
DS & 0.80 \\
SA & 0.74 \\
SS & 0.76 \\
\hline
\end{tabular}

Note. 'RP' refers to Reappraisal; 'SP' refers to Suppression; 'DS' refers to Distraction; 'SA' refers to Selective Attention; 'SS' refers to Situation Selection. Reappraisal and Suppression items were originally developed by Gross \& John (2003). ' $r$ ' refers to a Pearson correlation coefficient calculated between the test and retest observations. The sample was comprised of 156 individuals who completed the E-ERQ on MTurk approximately two weeks apart.

Supplementary Table 8. Correlations among E-ERQ strategies across all samples

\begin{tabular}{c|ccccc}
\hline ONLINE1 & RP & SP & DS & SA & SS \\
\hline RP & 1 & {$[-0.24,0.01]$} & {$[0.56,0.70]$} & {$[0.34,0.51]$} & {$[0.59,0.74]$}
\end{tabular}


70 EMOTION REGULATION TYPES

\begin{tabular}{c|ccccc} 
SP & -0.11 & 1 & {$[-0.02,0.22]$} & {$[0.01,0.24]$} & {$[-0.24,0.00]$} \\
DS & 0.63 & 0.12 & 1 & {$[0.37,0.54]$} & {$[0.51,0.66]$} \\
SA & 0.43 & 0.14 & 0.47 & 1 & {$[0.37,0.53]$} \\
SS & 0.67 & -0.12 & 0.59 & 0.45 & 1 \\
\hline ONLINE2 & RP & SP & DS & SA & SS \\
\hline RP & 1 & {$[-0.19,0.05]$} & {$[0.56,0.73]$} & {$[0.37,0.55]$} & {$[0.50,0.67]$} \\
SP & -0.08 & 1 & {$[0.03,0.25]$} & {$[0.12,0.23]$} & {$[-0.15,0.07]$} \\
DS & 0.65 & 0.14 & 1 & {$[0.46,0.60]$} & {$[0.43,0.64]$} \\
SA & 0.47 & 0.22 & 0.54 & 1 & {$[0.28,0.49]$} \\
SS & 0.60 & -0.05 & 0.54 & 0.40 & 1 \\
\hline ONLINE3 & RP & SP & DS & SA & SS \\
\hline RP & 1 & {$[-0.08,0.22]$} & {$[0.46,0.66]$} & {$[0.15,0.41]$} & {$[0.40,0.62]$} \\
SP & 0.07 & 1 & {$[0.15,0.39]$} & {$[0.29,0.51]$} & {$[-0.09,0.17]$} \\
DS & 0.57 & 0.27 & 1 & {$[0.31,0.53]$} & {$[0.26,0.51]$} \\
SA & 0.28 & 0.40 & 0.43 & 1 & {$[0.16,0.37]$} \\
SS & 0.52 & 0.04 & 0.39 & 0.27 & 1 \\
\hline LAB1 & RP & SP & DS & SA & SS \\
\hline RP & 1 & {$[-0.28,0.06]$} & {$[-0.06,0.41]$} & {$[-0.13,0.23]$} & {$[0.11,0.44]$} \\
SP & -0.12 & 1 & {$[0.14,0.45]$} & {$[0.22,0.54]$} & {$[-0.22,0.07]$} \\
DS & 0.18 & 0.29 & 1 & {$[0.21,0.50]$} & {$[0.10,0.43]$} \\
SA & 0.07 & 0.38 & 0.37 & 1 & {$[-0.12,0.19]$} \\
SS & 0.28 & -0.06 & 0.27 & 0.04 & 1 \\
\hline LAB2 & RP & SP & DS & SA & SS \\
\hline RP & 1 & {$[-0.26,0.16]$} & {$[0.18,0.50]$} & {$[0.12,0.47]$} & {$[0.46,0.78]$} \\
SP & -0.04 & 1 & {$[0.21,0.55]$} & {$[0.32,0.67]$} & {$[-0.30,0.09]$} \\
DS & 0.35 & 0.39 & 1 & {$[0.34,0.64]$} & {$[-0.01,0.38]$} \\
SA & 0.30 & 0.51 & 0.51 & 1 & {$[0.01,0.40]$} \\
SS & 0.64 & -0.11 & 0.19 & 0.20 & 1 \\
\hline TEST-RETEST1 & RP & SP & DS & SA & SS \\
\hline RP & 1 & {$[-0.23,0.08]$} & {$[0.56,0.75]$} & {$[0.23,0.51]$} & {$[0.48,0.70]$} \\
SP & -0.07 & 1 & {$[-0.02,0.26]$} & {$[0.19,0.47]$} & {$[-0.08,0.21]$} \\
DS & 0.67 & 0.12 & 1 & {$[0.40,0.65]$} & {$[0.54,0.73]$} \\
& & & & & \\
\hline & & & & 19 & 1 \\
\hline
\end{tabular}


71 EMOTION REGULATION TYPES

\begin{tabular}{c|ccccc} 
SA & 0.37 & 0.33 & 0.54 & 1 & {$[0.35,0.60]$} \\
SS & 0.60 & 0.07 & 0.64 & 0.49 & 1 \\
\hline TEST-RETEST2 & RP & SP & DS & SA & SS \\
\hline RP & 1 & {$[-0.33,0.03]$} & {$[0.54,0.76]$} & {$[0.12,0.46]$} & {$[0.44,0.71]$} \\
SP & -0.16 & 1 & {$[-0.14,0.20]$} & {$[0.13,0.46]$} & {$[-0.19,0.18]$} \\
DS & 0.67 & 0.02 & 1 & {$[0.36,0.63]$} & {$[0.52,0.76]$} \\
SA & 0.29 & 0.30 & 0.49 & 1 & {$[0.37,0.64]$} \\
SS & 0.59 & -0.01 & 0.64 & 0.50 & 1 \\
\hline
\end{tabular}

Note. Pearson's correlation values are reported in lower off-diagonal elements; 95\% confidence intervals are reported in the upper off-diagonal elements. 'RP' refers to Reappraisal; 'SP' refers to Suppression; 'DS' refers to Distraction; 'SA' refers to Selective Attention; 'SS' refers to Situation Selection. ONLINE1 $N=401$; ONLINE2 $N=406$; ONLINE3 $N=300$; LAB1 $N=127$; LAB2 $N=101$; TEST-RETEST1 $N=202$; TEST-RETEST2 $N=158$.

Supplementary Table 9. Cluster means across study.

ONLINE1

RP, SP, DS, SA, SS
ONLINE2

RP, SP, DS, SA, SS
ONLINE3

RP, SP, DS, SA, SS
LAB1

RP, SP, DS, SA, SS
LAB2

RP, SP, DS, SA, SS 


\begin{tabular}{l|l|l|l|l|l|}
\cline { 2 - 5 } Hi & $5.6,5.2,5.6,4.7,5.2$ & $5.6,5.1,5.6,4.7,5.3$ & $4.9,4.9,5.0,4.2,4.8$ & $5.3,4.3,5.6,4.5,5.8$ & $5.7,4.5,5.7,5.0,5.5$ \\
Lo & $4.0,3.9,4.0,3.0,3.7$ & $4.0,3.7,4.0,2.9,3.6$ & $3.9,3.1,3.7,2.5,3.9$ & $3.7,3.3,3.6,2.8,4.1$ & $4.4,4.1,4.1,3.1,4.2$ \\
Mix & $5.7,2.8,5.1,4.1,5.5$ & $5.9,2.5,5.4,3.9,5.5$ & $5.6,3.0,5.1,3.3,5.6$ & $5.3,2.8,4.6,2.6,5.3$ & $5.4,2.1,3.6,2.6,5.6$ \\
\hline
\end{tabular}

Note. 'RP' refers to Reappraisal; 'SP' refers to Suppression; 'DS' refers to Distraction; 'SA' refers to Selective Attention; 'SS' refers to Situation Selection. Strategy usage assessments reflect mean scores from the novel E-ERQ. ONLINE1 and ONLINE2 were collected via MTurk, ONLINE 3 was collected via UCLA's subject pool for online studies, LAB1 and LAB2 were laboratory studies whose participants were recruited from the UCLA undergraduate student community. Entries represent the average usage for a given strategy for all individuals within a given cluster, within a given sample.

Supplementary Table 10. Aggregate effect size estimates of group differences between clusters on mental health symptoms (based on affective symptoms). 


\begin{tabular}{cccc}
\hline & Mix $-\mathrm{Hi}$ & Mix - Lo & $\mathrm{Hi}-$ Lo \\
\hline Positive Affect & $\mathbf{0 . 4 1 2}[\mathbf{0 . 1 1 0 , 0 . 7 1 3}]$ & $\mathbf{0 . 6 5 4}[\mathbf{0 . 3 3 2 , 0 . 9 7 1}]$ & $0.234[-0.087,0.553]$ \\
Negative Affect & $-0.288[-0.586,0.011]$ & $\mathbf{- 0 . 4 7 8}[-\mathbf{- 0 . 7 8 8 , - 0 . 1 6 5}]$ & $-0.198[-0.516,0.121]$ \\
Positive Expressivity & $\mathbf{0 . 8 9 2}[\mathbf{0 . 5 6 8}, \mathbf{1 . 2 1 3}]$ & $\mathbf{0 . 6 3 6}[\mathbf{0 . 3 1 6 , 0 . 9 5 4}]$ & $-0.190[-0.509,0.130]$ \\
Negative Expressivity & $\mathbf{0 . 9 1 6}[\mathbf{0 . 5 8 7}, \mathbf{1 . 2 4 0}]$ & $0.131[-0.176,0.438]$ & $\mathbf{- 0 . 7 5 9}[-\mathbf{- 1 . 0 9 7 , - \mathbf { 0 . 4 1 5 } ]}$ \\
\hline
\end{tabular}

Note. Bolded entries indicate effect sizes whose confidence intervals do not include zero. 'Positive Affect' and 'Negative Affect' refer to trait positive and negative affect assessed via the PANAS; Expressivity metrics were assessed by the BEQ. 'Hi' refers to the cluster of participants who tended to frequently use all five emotion regulation strategies; 'Lo' refers to the cluster of participants who tended to infrequently use all five strategies; 'Mix' refers to the cluster of participants who selectively moderated strategy usage. Sign of comparison indicated by column title. Entries into the table reflect averages of Cohen's $d$ estimates all appropriate samples. Entries in brackets reflect aggregated analytic $95 \%$ confidence intervals, averaged over all appropriate samples. LAB2 data are not represented in these data as these metrics were not collected in that sample.

Supplementary Table 11. Effect size estimates of group differences between clusters on mental health symptoms (affective symptoms) across four samples 


\begin{tabular}{|c|c|c|c|}
\hline & Mix - Hi & Mix - Lo & $\mathrm{Hi}-\mathrm{Lo}$ \\
\hline \multicolumn{4}{|c|}{ ONLINE1 } \\
\hline Positive Affect & $0.397[0.150,0.643]$ & $0.905[0.646,1.162]$ & $0.454[0.201,0.706]$ \\
\hline Negative Affect & $-0.242[-0.485,0.002]$ & $-0.515[-0.756,-0.272]$ & $-0.293[-0.542,0.044]$ \\
\hline Positive Expressivity & $0.967[0.694,1.236]$ & $0.931[0.670,1.189]$ & $0.032[-0.214,0.278]$ \\
\hline Negative Expressivity & $0.998[0.724,1.269]$ & $0.327[0.089,0.564]$ & $-0.569[-0.824,-0.312]$ \\
\hline \multicolumn{4}{|c|}{ ONLINE2 } \\
\hline Positive Affect & $0.504[0.256,0.751]$ & $0.844[0.584,1.101]$ & $0.323[0.084,0.561]$ \\
\hline Negative Affect & $-0.198[-0.439,0.043]$ & $-0.504[-0.749,-0.256]$ & $-0.311[-0.549,-0.072]$ \\
\hline Positive Expressivity & $1.224[0.941,1.503]$ & $1.101[0.827,1.371]$ & $-0.046[-0.282,-0.189]$ \\
\hline Negative Expressivity & $1.174[0.895,1.451]$ & $0.587[0.337,0.834]$ & $-0.558[-0.801,-0.312]$ \\
\hline \multicolumn{4}{|c|}{ ONLINE3 } \\
\hline Positive Affect & $0.539[0.250,0.826]$ & $0.437[0.136,0.736]$ & $-0.074[-0.349,0.201]$ \\
\hline Negative Affect & $-0.480[-0.765,-0.193]$ & $-0.550[-0.852,-0.244]$ & $-0.084[-0.359,0.191]$ \\
\hline Positive Expressivity & $0.969[0.661,1.273]$ & $0.329[0.031,0.625]$ & $-0.550[-0.833,-0.265]$ \\
\hline Negative Expressivity & $0.734[0.437,1.027]$ & $-0.297[-0.593,-0.000]$ & $-0.988[-1.289,0.683]$ \\
\hline \multicolumn{4}{|c|}{ [ 10} \\
\hline Positive Affect & $0.208[-0.217,0.630]$ & $0.429[-0.036,0.885]$ & $0.233[-0.285,0.746]$ \\
\hline Negative Affect & $-0.234[-0.656,0.192]$ & $-0.344[-0.797,0.114]$ & $-0.104[-0.616,0.409]$ \\
\hline Positive Expressivity & $0.409[-0.027,0.834]$ & $0.185[-0.266,0.632]$ & $-0.196[-0.709,0.320]$ \\
\hline Negative Expressivity & $0.759[0.293,1.214]$ & $-0.092[-0.538,0.355]$ & $-0.922[-1.477,-0.355]$ \\
\hline
\end{tabular}

Note. 'Positive Affect' and 'Negative Affect' refer to trait positive and negative affect assessed via the PANAS; Expressivity metrics were assessed by the BEQ. 'Hi' refers to the cluster of participants who tended to frequently use all five emotion regulation strategies; 'Lo' refers to the cluster of participants who tended to infrequently use all five strategies; 'Mix' refers to the cluster of participants who selectively moderated strategy usage. Sign of comparison indicated by column title. Entries into the table reflect averages of Cohen's $d$ estimates all appropriate samples. Entries in brackets reflect analytic 95\% confidence intervals, averaged over all appropriate samples. LAB2 data are only represented in the sensation seeking effect sizes, as the other affective trait assessments were not administered in that sample.

\begin{tabular}{cccc}
\hline & Mix $-\mathrm{Hi}$ & $\mathrm{Mix}-\mathrm{Lo}$ & $\mathrm{Hi}-\mathrm{Lo}$ \\
\hline Anxiety & $\mathbf{- 0 . 3 6 3}[\mathbf{- 0 . 7 1 0 , - \mathbf { 0 . 0 1 1 } ]}$ & $-0.292[-0.636,0.056]$ & $0.072[-0.276,0.419]$ \\
Depression & $\mathbf{- 0 . 5 2 5}[\mathbf{- 0 . 8 7 6 , - 0 . 1 7 1}]$ & $\mathbf{- 0 . 6 6 0}[\mathbf{- 1 . 0 1 2}, \mathbf{- 0 . 2 9 7}]$ & $-0.121[-0.467,0.226]$ \\
General Distress & $\mathbf{- 0 . 4 0 1}[\mathbf{- 0 . 7 0 2}, \mathbf{- 0 . 0 9 7}]$ & $\mathbf{- 0 . 5 7 6}[\mathbf{- 0 . 8 9 2 ,}, \mathbf{- 0 . 2 5 5}]$ & $-0.169[-0.487,0.150]$
\end{tabular}


Aggregate effect size estimates of group differences between clusters on mental health symptoms (clinical symptoms).

Note. 'Anxiety' refers to scores from the anxious arousal subscale of the Mini-MASQ for ONLINE1-3 and LAB1, and scores from the generalized anxiety subscale of the SCAARED for LAB2; 'Depression' refers to scores from the anhedonic depression subscale of the Mini-MASQ for ONELINE1-3. 'General Distress' refers to scores from the generalized internalizing symptomatology subscale of the MINI-MASQ for ONLINE13 and LAB1. 'Perceived Stress' refers to scores from the PSS for ONLINE1-3 and LAB1. 'Hi' refers to the cluster of participants who tended to frequently use all five emotion regulation strategies; 'Lo' refers to the cluster of participants who tended to infrequently use all five strategies; 'Mix' refers to the cluster of participants who selectively moderated strategy usage. Sign of comparison indicated by column title. Entries into the table reflect averages of Cohen's $d$ estimates all appropriate samples. Entries in brackets reflect aggregated analytic 95\% confidence intervals, averaged over all appropriate samples. 
Supplementary Table 13. Effect size estimates of group differences between clusters on mental health symptoms (clinical symptoms) across five samples.

\begin{tabular}{|c|c|c|c|}
\hline & $\mathrm{Mix}-\mathrm{Hi}$ & Mix - Lo & $\mathrm{Hi}-\mathrm{Lo}$ \\
\hline \multicolumn{4}{|c|}{ ONLINE1 } \\
\hline Anxiety & $-0.136[-0.378,0.106]$ & $-0.329[-0.566,-0.091]$ & $-0.218[-0.465,0.030]$ \\
\hline Depression & $-0.547[-0.797,-0.294]$ & $-0.918[-1.175,-0.658]$ & $-0.315[-0.563,-0.065]$ \\
\hline General Distress & $-0.407[-0.654,-0.160]$ & $-0.654[-0.900,-0.406]$ & $-0.261[-0.508,-0.012]$ \\
\hline Perceived Stress & $-0.467[-0.715,-0.218]$ & $-0.767[-1.017,-0.515]$ & $-0.337[-0.586,-0.086]$ \\
\hline \multicolumn{4}{|c|}{ ONLINE2 } \\
\hline Anxiety & $-0.311[-0.553,-0.067]$ & $-0.418[-0.661,-0.173]$ & $-0.070[-0.305,0.166]$ \\
\hline Depression & $-0.605[-0.855,-0.353]$ & $-0.841[-1.098,-0.581]$ & $-0.235[-0.472,0.002]$ \\
\hline General Distress & $-0.349[-0.592,-0.104]$ & $-0.604[-0.852,-0.353]$ & $-0.283[-0.520,-0.045]$ \\
\hline Perceived Stress & $-0.441[-0.687,-0.194]$ & $-0.546[-0.793,-0.298]$ & $-0.134[-0.369,0.103]$ \\
\hline \multicolumn{4}{|c|}{ ONLINE3 } \\
\hline Anxiety & $-0.382[-0.665,-0.098]$ & $-0.200[-0.494,0.096]$ & $0.198[-0.078,0.474]$ \\
\hline Depression & $-0.725[-1.018,-0.429]$ & $-0.418[-0.716,-0.118]$ & $0.278[-0.000,0.554]$ \\
\hline General Distress & $-0.389[-0.672,-0.105]$ & $-0.378[-0.675,-0.079]$ & $-0.009[-0.284,0.265]$ \\
\hline Perceived Stress & $-0.409[-0.692,-0.124]$ & $-0.529[-0.830,-0.224]$ & $-0.145[-0.420,0.130]$ \\
\hline \multicolumn{4}{|c|}{ LAB1 } \\
\hline Anxiety & $-0.279[-0.702,0.149]$ & $-0.331[-0.783,0.127]$ & $-0.016[-0.528,0.496]$ \\
\hline Depression & $-0.295[-0.720,0.133]$ & $-0.631[-1.103,-0.148]$ & $-0.294[-0.810,0.225]$ \\
\hline General Distress & $-0.458[-0.889,-0.019]$ & $-0.667[-1.142,-0.181]$ & $-0.123[-0.635,0.391]$ \\
\hline Perceived Stress & $-0.422[-0.852,0.014]$ & $-0.733[-1.215,-0.239]$ & $-0.282[-0.796,0.237]$ \\
\hline \multicolumn{4}{|c|}{ LAB2 } \\
\hline Anxiety & $-0.704[-1.253,-0.146]$ & $-0.180[-0.678,0.320]$ & $0.465[-0.003,0.927]$ \\
\hline Depression & $-0.455[-0.989,0.086]$ & $-0.492[-0.998,0.018]$ & $-0.039[-0.490,0.413]$ \\
\hline
\end{tabular}

refers to scores from the anxious arousal subscale of the Mini-MASQ for ONLINE1-3 and LAB1, and scores from the generalized anxiety subscale of the SCAARED for LAB2; 'Depression' refers to scores from the anhedonic depression subscale of the Mini-MASQ for ONELINE1-3. 'General Distress' refers to scores from the generalized internalizing symptomatology subscale of the MINI-MASQ for ONLINE1-3 and LAB1. 'Perceived Stress' refers to scores from the PSS for ONLINE1-3 and LAB1. 'Hi' refers to the cluster of participants who tended to frequently use all five emotion regulation strategies; 'Lo' refers to the cluster of participants who tended to infrequently use all five strategies; 'Mix' refers to the cluster of participants who selectively moderated strategy usage. Sign of comparison indicated by column title. Entries into the table reflect Cohen's $d$ estimates. Entries in brackets reflect analytic 95\% confidence intervals. 
Supplementary Table 14. Ridge regression coefficients quantifying associations between emotion regulation strategies and mental health symptoms (affective symptoms).

\begin{tabular}{|c|c|c|c|c|}
\hline & Positive Affect & Negative Affect & Positive Expressivity & Negative Expressivity \\
\hline RP & $0.240[0.148,0.333]$ & $-0.249[-0.359,-0.140]$ & $0.127[-0.007,0.260]$ & $-0.235[-0.392,-0.079]$ \\
\hline SP & $-0.122[-0.200,-0.044]$ & $0.043[-0.032,0.117]$ & $-0.564[-0.669,-0.459]$ & $-0.748[-0.883,-0.613]$ \\
\hline DS & $-0.099[-0.191,-0.007]$ & $0.121[0.030,0.213]$ & $0.075[-0.052,0.203]$ & $0.148[-0.006,0.301]$ \\
\hline SA & $0.012[-0.066,0.091]$ & $-0.004[-0.079,0.071]$ & $-0.001[-0.106,0.105]$ & $0.036[-0.091,0.163]$ \\
\hline SS & $0.121[0.030,0.213]$ & $-0.013[-0.103,0.077]$ & $0.113[-0.011,0.237]$ & $-0.007[-0.147,0.133]$ \\
\hline
\end{tabular}

Note. 'Positive Affect' and 'Negative Affect' refer to trait positive and negative affect assessed via the PANAS; Expressivity metrics were assessed by the BEQ. 'RP' refers to Reappraisal; 'SP' refers to Suppression; 'DS' refers to Distraction; 'SA' refers to Selective Attention; 'SS' refers to Situation Selection. Strategy usage assessments reflect mean scores from the novel E-ERQ. Entries in brackets reflect aggregated percentile bootstrapped 95\% confidence intervals, averaged over all appropriate samples. Coefficients were also averaged estimates from all available samples. LAB2 data are not represented in these data as these metrics were not collected in that sample. 
Supplementary Table 15. Ridge regression coefficients quantifying associations between emotion regulation strategies and mental health symptoms (affective symptoms) across four samples.

\begin{tabular}{|c|c|c|c|c|}
\hline & Positive Affect & Negative Affect & Positive Expressivity & Negative Expressivity \\
\hline \multicolumn{5}{|c|}{ ONLINE1 } \\
\hline RP & $0.288[0.182,0.393]$ & $-0.253[-0.353,-0.153]$ & $0.208[0.079,0.337]$ & $-0.234[-0.381,-0.086]$ \\
\hline SP & $-0.108[-0.183,-0.032]$ & $0.004[-0.060,0.067]$ & $-0.671[-0.775,-0.566]$ & $-0.791[-0.902,-0.679]$ \\
\hline DS & $-0.188[-0.287,-0.088]$ & $0.150[0.065,0.234]$ & $0.082[-0.049,0.213]$ & $0.232[0.092,0.372]$ \\
\hline SA & $0.100[0.018,0.181]$ & $-0.006[-0.077,0.065]$ & $0.064[-0.046,0.173]$ & $0.041[-0.072,0.153]$ \\
\hline SS & $0.225[0.117,0.333]$ & $-0.061[-0.153,0.031]$ & $0.210[0.088,0.333]$ & $-0.019[-0.167,0.129]$ \\
\hline \multicolumn{5}{|c|}{ ONLINE2 } \\
\hline $\mathrm{RP}$ & $0.336[0.232,0.441]$ & $0.048[-0.022,0.119]$ & $0.192[0.045,0.338]$ & $-0.257[-0.398,-0.115]$ \\
\hline SP & $-0.158[-0.239,-0.077]$ & $0.262[0.157,0.368]$ & $-0.738[-0.849,-0.628]$ & $-0.820[-0.944,-0.696]$ \\
\hline DS & $-0.150[-0.249,-0.052]$ & $-0.017[-0.085,0.052]$ & $0.066[-0.052,0.185]$ & $0.180[0.042,0.318]$ \\
\hline SA & $0.024[-0.066,0.113]$ & $-0.021[-0.118,0.076]$ & $0.051[-0.051,0.153]$ & $0.110[-0.006,0.227]$ \\
\hline SS & $0.114[0.019,0.208]$ & $-0.198[-0.285,-0.111]$ & $0.152[0.007,0.297]$ & $0.067[-0.061,0.195]$ \\
\hline \multicolumn{5}{|c|}{ ONLINE3 } \\
\hline RP & $0.170[0.090,0.250]$ & $0.040[-0.040,0.121]$ & $0.043[-0.081,0.167]$ & $-0.245[-0.399,-0.091]$ \\
\hline SP & $-0.129[-0.199,-0.059]$ & $0.063[-0.015,0.142]$ & $-0.531[-0.645,-0.418]$ & $-0.703[-0.838,-0.568]$ \\
\hline DS & $-0.065[-0.138,0.008]$ & $0.052[-0.024,0.129]$ & $0.132[-0.006,0.270]$ & $0.103[-0.048,0.254]$ \\
\hline SA & $-0.003[-0.069,0.062]$ & $-0.028[-0.112,0.055]$ & $-0.058[-0.168,0.053]$ & $0.050[-0.079,0.178]$ \\
\hline SS & $0.104[0.027,0.182]$ & $-0.190[-0.333,-0.047]$ & $0.056[-0.052,0.165]$ & $-0.036[-0.166,0.094]$ \\
\hline \multicolumn{5}{|c|}{ LAB1 } \\
\hline $\mathrm{RP}$ & $0.167[0.087,0.247]$ & $0.078[-0.005,0.161]$ & $0.063[-0.070,0.196]$ & $-0.205[-0.389,-0.022]$ \\
\hline SP & $-0.092[-0.177,-0.007]$ & $0.010[-0.088,0.107]$ & $-0.315[-0.408,-0.222]$ & $-0.678[-0.847,-0.508]$ \\
\hline DS & $0.006[-0.091,0.103]$ & $-0.046[-0.131,0.038]$ & $0.021[-0.102,0.145]$ & $0.076[-0.109,0.260]$ \\
\hline SA & $-0.072[-0.149,0.006]$ & $0.058[-0.028,0.145]$ & $-0.059[-0.158,0.039]$ & $-0.056[-0.205,0.093]$ \\
\hline SS & $0.041[-0.044,0.127]$ & $0.078[-0.005,0.161]$ & $0.033[-0.089,0.155]$ & $-0.040[-0.196,0.115]$ \\
\hline
\end{tabular}

Note. 'Positive Affect' and 'Negative Affect' refer to trait positive and negative affect assessed via the PANAS; Expressivity metrics were assessed by the BEQ. 'RP' refers to Reappraisal; 'SP' refers to Suppression; 'DS' refers to Distraction; 'SA' refers to Selective Attention; 'SS' refers to Situation Selection. Strategy usage assessments reflect mean scores from the novel E-ERQ. Entries in brackets reflect percentile bootstrapped $95 \%$ confidence intervals 
Supplementary Table 16. Ridge regression coefficients quantifying associations between emotion regulation strategies and on mental health symptoms (based on clinical symptoms).

\begin{tabular}{c|cccc}
\hline & Anxiety & Depression & General Distress & Perceived Stress \\
\hline RP & $\mathbf{- 0 . 1 0 9}[-\mathbf{- 0 . 1 7 9 ,}, \mathbf{- 0 . 0 3 9}]$ & $\mathbf{- 0 . 2 9 8}[-\mathbf{- 0 . 4 1 3}, \mathbf{- 0 . 1 8 4}]$ & $\mathbf{- 0 . 3 3 9}[-\mathbf{- 0 . 4 6 4 , - 0 . 2 1 4}]$ & $\mathbf{- 0 . 3 3 1}[-\mathbf{- 0 . 4 2 1 ,} \mathbf{- 0 . 2 4 2}]$ \\
SP & $0.041[-0.016,0.098]$ & $\mathbf{0 . 1 7 7}[\mathbf{0 . 0 8 4}, \mathbf{0 . 2 6 9}]$ & $0.095[-0.002,0.191]$ & $0.053[-0.019,0.124]$ \\
DS & $0.053[-0.007,0.113]$ & $0.107[-0.003,0.217]$ & $\mathbf{0 . 1 8 1}[\mathbf{0 . 0 6 6}, \mathbf{0 . 2 9 7}]$ & $\mathbf{0 . 1 9 9}[\mathbf{0 . 1 0 8 , 0 . 2 8 9}]$ \\
SA & $0.035[-0.026,0.096]$ & $0.002[-0.099,0.103]$ & $-0.008[-0.108,0.091]$ & $-0.002[-0.076,0.072]$ \\
SS & $-0.002[-0.070,0.066]$ & $-0.068[-0.172,0.036]$ & $-0.043[-0.152,0.066]$ & $-0.029[-0.110,0.053]$ \\
\hline
\end{tabular}

Note. 'Anxiety' refers to scores from the anxious arousal subscale of the Mini-MASQ for ONLINE1-3 and LAB1, and scores from the generalized anxiety subscale of the SCAARED for LAB2; 'Depression' refers to scores from the anhedonic depression subscale of the Mini-MASQ for ONELINE1-3. 'General Distress' refers to scores from the generalized internalizing symptomatology subscale of the MINI-MASQ for ONLINE13 and LAB1. 'Perceived Stress' refers to scores from the PSS for ONLINE1-3 and LAB1. 'RP' refers to Reappraisal; 'SP' refers to Suppression; 'DS' refers to Distraction; 'SA' refers to Selective Attention; 'SS' refers to Situation Selection. Strategy usage assessments reflect mean scores from the novel E-ERQ. Entries in brackets reflect percentile bootstrapped 95\% confidence intervals, averaged over all appropriate samples. Coefficients were also averaged estimates from all available samples. 
Supplementary Table 17. Ridge regression coefficients quantifying associations between emotion regulation strategies and mental health symptoms (clinical symptoms) across five samples.

\begin{tabular}{|c|c|c|c|c|}
\hline & Anxiety & Depression & General Distress & Perceived Stress \\
\hline & \multicolumn{4}{|c|}{ ONLINE1 } \\
\hline $\mathrm{RP}$ & $-0.162[-0.230,-0.094]$ & $-0.339[-0.450,-0.227]$ & $-0.374[-0.488,-0.261]$ & $-0.406[-0.500,-0.312]$ \\
\hline SP & $0.010[-0.033,0.052]$ & $0.207[0.124,0.290]$ & $0.073[-0.014,0.160]$ & $0.024[-0.051,0.100]$ \\
\hline DS & $0.023[-0.038,0.084]$ & $0.167[0.072,0.261]$ & $0.257[0.151,0.362]$ & $0.299[0.202,0.395]$ \\
\hline SA & $0.090[0.031,0.149]$ & $-0.121[-0.213,-0.029]$ & $-0.017[-0.113,0.080]$ & $0.016[-0.060,0.093]$ \\
\hline \multirow[t]{2}{*}{ SS } & $-0.038[-0.112,0.035]$ & $-0.112[-0.231,0.007]$ & $-0.147[-0.270,-0.025]$ & $-0.121[-0.228,-0.014]$ \\
\hline & \multicolumn{4}{|c|}{ ONLINE2 } \\
\hline $\mathrm{RP}$ & $-0.166[-0.261,-0.071]$ & $-0.406[-0.524,-0.289]$ & $-0.514[-0.652,-0.377]$ & $-0.447[-0.547,-0.346]$ \\
\hline SP & $0.052[0.000,0.104]$ & $0.182[0.093,0.271]$ & $0.118[0.025,0.211]$ & $0.076[-0.001,0.153]$ \\
\hline DS & $0.091[0.026,0.156]$ & $0.272[0.143,0.401]$ & $0.326[0.193,0.458]$ & $0.308[0.197,0.420]$ \\
\hline SA & $0.041[-0.013,0.096]$ & $-0.019[-0.121,0.083]$ & $0.024[-0.068,0.116]$ & $0.006[-0.078,0.090]$ \\
\hline \multirow[t]{2}{*}{ SS } & $0.016[-0.071,0.094]$ & $-0.140[-0.242,-0.038]$ & $-0.052[-0.167,0.063]$ & $-0.003[-0.094,0.087]$ \\
\hline & \multicolumn{4}{|c|}{ ONLINE3 } \\
\hline $\mathrm{RP}$ & $-0.054[-0.110,0.001]$ & $-0.264[-0.367,-0.162]$ & $-0.172[-0.265,-0.079]$ & $-0.281[-0.365,-0.196]$ \\
\hline SP & $0.045[-0.008,0.099]$ & $0.150[0.059,0.242]$ & $0.059[-0.025,0.144]$ & $0.026[-0.043,0.096]$ \\
\hline DS & $0.013[-0.039,0.065]$ & $0.078[-0.023,0.179]$ & $0.060[-0.036,0.156]$ & $0.136[0.053,0.219]$ \\
\hline SA & $0.064[0.006,0.122]$ & $0.077[-0.012,0.165]$ & $0.011[-0.075,0.098]$ & $-0.006[-0.073,0.061]$ \\
\hline \multirow[t]{2}{*}{ SS } & $-0.001[-0.050,0.049]$ & $-0.078[-0.166,0.011]$ & $-0.035[-0.129,0.059]$ & $-0.018[-0.088,0.052]$ \\
\hline & \multicolumn{4}{|c|}{ LAB1 } \\
\hline $\mathrm{RP}$ & $-0.062[-0.122,-0.002]$ & $-0.247[-0.365,-0.128]$ & $-0.295[-0.451,-0.140]$ & $-0.192[-0.269,-0.114]$ \\
\hline SP & $0.057[-0.003,0.116]$ & $0.226[0.124,0.328]$ & $0.128[0.005,0.251]$ & $0.084[0.019,0.148]$ \\
\hline DS & $0.030[-0.021,0.080]$ & $-0.022[-0.146,0.103]$ & $0.084[-0.045,0.212]$ & $0.052[-0.021,0.125]$ \\
\hline SA & $-0.033[-0.083,0.017]$ & $-0.031[-0.137,0.075]$ & $-0.051[-0.175,0.072]$ & $-0.024[-0.092,0.043]$ \\
\hline \multirow[t]{2}{*}{ SS } & $-0.002[-0.046,0.042]$ & $-0.021[-0.129,0.087]$ & $0.062[-0.042,0.164]$ & $0.027[-0.032,0.085]$ \\
\hline & \multicolumn{4}{|c|}{ LAB2 } \\
\hline RP & $-0.101[-0.173,-0.028]$ & $-0.236[-0.358,-0.115]$ & - & - \\
\hline SP & $0.042[-0.034,0.118]$ & $0.120[0.022,0.217]$ & - & - \\
\hline DS & $0.107[0.034,0.180]$ & $0.039[-0.060,0.139]$ & - & - \\
\hline SA & $0.013[-0.071,0.098]$ & $0.104[-0.015,0.223]$ & - & - \\
\hline SS & $0.021[-0.069,0.112]$ & $0.011[-0.090,0.112]$ & - & - \\
\hline
\end{tabular}


Note. 'Anxiety' refers to scores from the anxious arousal subscale of the Mini-MASQ for ONLINE1-3 and LAB1, and scores from the generalized anxiety subscale of the SCAARED for LAB2; 'Depression' refers to scores from the anhedonic depression subscale of the Mini-MASQ for ONELINE1-3. 'General Distress' refers to scores from the generalized internalizing symptomatology subscale of the MINI-MASQ for ONLINE13 and LAB1. 'Perceived Stress' refers to scores from the PSS for ONLINE1-3 and LAB1. 'RP' refers to Reappraisal; 'SP' refers to Suppression; 'DS' refers to Distraction; 'SA' refers to Selective Attention; 'SS' refers to Situation Selection. Strategy usage assessments reflect mean scores from the novel E-ERQ. Entries in brackets reflect percentile bootstrapped 95\% confidence intervals. 


\section{EMOTION REGULATION TYPES}

Supplementary Table 18. Growth curve modeling results predicting longitudinal anxiety measurements from cluster membership

\begin{tabular}{c|c}
\hline & Anxiety \\
\hline Intercept & $1.170(0.141)^{* * *}$ \\
Time (Days) & $-0.001(0.000)$ \\
Sex & $0.242(0.115)^{*}$ \\
Quarter Start & $-0.432(0.176)^{*}$ \\
Any Covid & $0.146(0.158)$ \\
Hi & $0.116(0.108)$ \\
Mix & $-0.149(0.117)$ \\
$\operatorname{SD}\left(e_{i t}\right)$ & 0.441 \\
$\operatorname{SD}\left(\pi_{0 i}\right)$ & 0.002 \\
$\operatorname{SD}\left(\pi_{l i}\right)$ & 0.193 \\
$\operatorname{Cor}\left(\pi_{0 i}, \pi_{l i}\right)$ & -0.057 \\
$\mathrm{AIC}$ & 209.046 \\
$\mathrm{BIC}$ & 252.834 \\
\hline
\end{tabular}

Note. $+p<.10,{ }^{*} p<.05, * * p<.01, * * * p<.001$; time was coded as days since baseline (zero $=$ day of baseline assessment); Sex was dummy coded $(0=$ male, $1=$ female); quarter start referred to the academic quarter that a given participant enrolled in (dummy coded $0=$ fall, $1=$ winter); Any COVID referred to whether a participant provided any data during any point during the COVID-19 pandemic (dummy coded $0=$ no data provided during COVID-19 pandemic; 1 = at least one follow-up data point collected during COVID-19 pandemic). 'Hi' and 'Mix' refer to clusters identified by our K-medoids analysis. SD refers to standard deviation of random effects or level 1 errors; Cor refers to correlations between conditional random effects; AIC/BIC refer to Akaike and Bayesian Information Criterion, respectively. 


\section{EMOTION REGULATION TYPES}

Supplementary Table 19. Growth curve modeling results predicting longitudinal anxiety measurements from individual differences in strategy usage

\begin{tabular}{|c|c|}
\hline & Anxiety \\
\hline Intercept & $1.213(0.296)^{* * *}$ \\
\hline Time (Days) & $-0.001(0.000)$ \\
\hline Sex & $0.278(0.112)^{*}$ \\
\hline Quarter Start & $-0.408(0.172)^{*}$ \\
\hline Any Covid & $0.124(0.157)$ \\
\hline $\mathrm{RP}$ & $-0.121(0.061)^{+}$ \\
\hline SP & $0.043(0.040)$ \\
\hline DS & $0.089(0.043)^{*}$ \\
\hline SA & $-0.007(0.042)$ \\
\hline SS & $0.000(0.051)$ \\
\hline $\mathrm{SD}\left(e_{i t}\right)$ & 0.191 \\
\hline $\operatorname{SD}\left(\pi_{0 i}\right)$ & 0.424 \\
\hline $\operatorname{SD}\left(\pi_{l i}\right)$ & 0.002 \\
\hline $\operatorname{Cor}\left(\pi_{0 i}, \pi_{l i}\right)$ & 0.009 \\
\hline AIC & 223.602 \\
\hline BIC & 278.178 \\
\hline
\end{tabular}

Note. $+p<.10,{ }^{*} p<.05, * * p<.01, * * * p<.001$; time was coded as days since baseline (zero $=$ day of baseline assessment); Sex was dummy coded $(0=$ male, $1=$ female); quarter start referred to the academic quarter that a given participant enrolled in (dummy coded $0=$ fall, $1=$ winter); Any COVID referred to whether a participant provided any data during any point during the COVID-19 pandemic (dummy coded $0=$ no data provided during COVID-19 pandemic; 1 = at least one follow-up data point collected during COVID-19 pandemic). 'RP' refers to Reappraisal; 'SP' refers to Suppression; 'DS' refers to Distraction; 'SA' refers to Selective Attention; 'SS' refers to Situation Selection. SD refers to standard deviation of random effects or level 1 errors; Cor refers to correlations between conditional random effects; AIC/BIC refer to Akaike and Bayesian Information Criterion, respectively. 